CRYSTALLOGRAPHIC COMMUNICATIONS

ISSN 2056-9890

Received 25 January 2017

Accepted 27 January 2017

Edited by J. Simpson, University of Otago, New Zealand

Keywords: crystal structure; rhodamine; xanthene; hydrogen bond; chiral sensors.

CCDC references: 1529967; 1529966; 1529965

Supporting information: this article has supporting information at journals.iucr.org/e

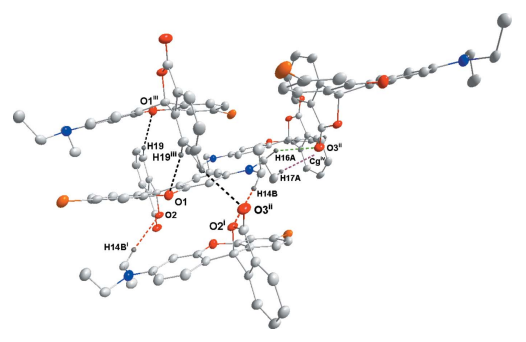

OPEN $\odot$ ACCESS

\section{Synthesis, resolution and crystal structures of two enantiomeric rhodamine derivatives}

\author{
Clifton J. Stephenson, ${ }^{\mathrm{a}}$ Joel T. Mague, ${ }^{\mathrm{b} *}$ Nathaniel Kamm, ${ }^{\mathrm{a}}$ Nathalie Aleman, \\ Dayla Rich, ${ }^{\text {a }}$ Quynh-Nhu Dang ${ }^{\mathrm{a}}$ and Ha Van Nguyen ${ }^{\mathrm{a}}$
}

aDepartment of Chemistry, Loyola University, New Orleans, LA 70118, USA, and ${ }^{\mathbf{b}}$ Department of Chemistry, Tulane
University, New Orleans, LA 70118, USA. *Correspondence e-mail: joelt@tulane.edu

The title molecule, rac-6'-bromo-3'-diethylamino-3H-spiro[2-benzofuran-1,9'xanthen]-3-one, $\mathrm{C}_{24} \mathrm{H}_{20} \mathrm{BrNO}_{3}$, was synthesized and the two enantiomers which formed were separated. The structures of all three compounds were determined and compared with those of a variety of related derivatives. A notable feature is the fold of the xanthene portion which ranges from $15.15(13)^{\circ}$ in the racemate to $2.42(2)^{\circ}$ in one molecule of the $R$ enantiomer with that for the $S$ enantiomer having an intermediate value. The differences are attributed to the number and severity of intermolecular interactions which include $\mathrm{C}-\mathrm{H} \cdots \mathrm{O}$ hydrogen bonds, $\mathrm{C}-\mathrm{H} \cdots \pi($ ring) and, in the $S$ enantiomer, a $\pi$-stacking interaction between the carbonyl group and an aromatic ring.

\section{Chemical context}

The compounds synthesized here are part of ongoing work to form chiral sensors based on the supramolecular interactions of chiral rhodamine derivatives with analytes. Enantiomeric sensing is critical for the efficient and safe formation of chiral pharmaceuticals (LaPlante et al., 2011) since enantiomers may have vastly different biological effects including toxicity (Reist et al., 1998). Most current methods for the detection of enantiomeric purity involve chromatographic techniques that require costly instrumentation (Wang et al., 2006). Chiral supramolecular sensors offer an inexpensive alternative (Chen et al., 2012; Jo et al., 2014; Zhang et al., 2014; Yu \& Pu, 2015). Supramolecular sensors, such as modified rhodamine derivatives, have garnered recent interest as sensors with biological applications (Pak et al., 2015; You et al., 2015). Additionally, recent work has shown that rhodamine B can function as a sensor differentiating between diastereomers (Shimizu \& Stephenson, 2010). Herein, we report the synthesis, resolution and structures of two asymmetric rhodamine derivatives $\mathbf{4}$ and 5 which are being investigated for potential as chiral sensors.

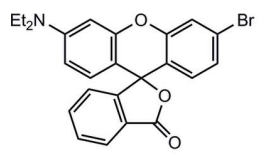

3

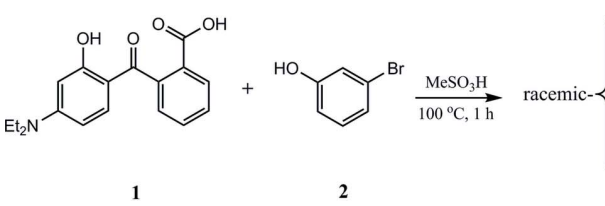

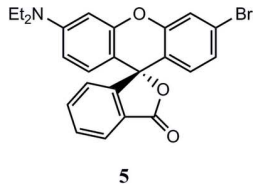

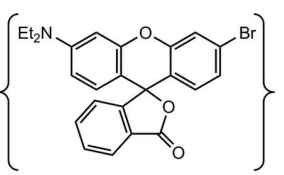

3 
Table 1

Dihedral angles $\left(^{\circ}\right)$ in selected rhodamine derivatives..

$R_{1}-R_{6}$ positions are defined in Fig. 1 .

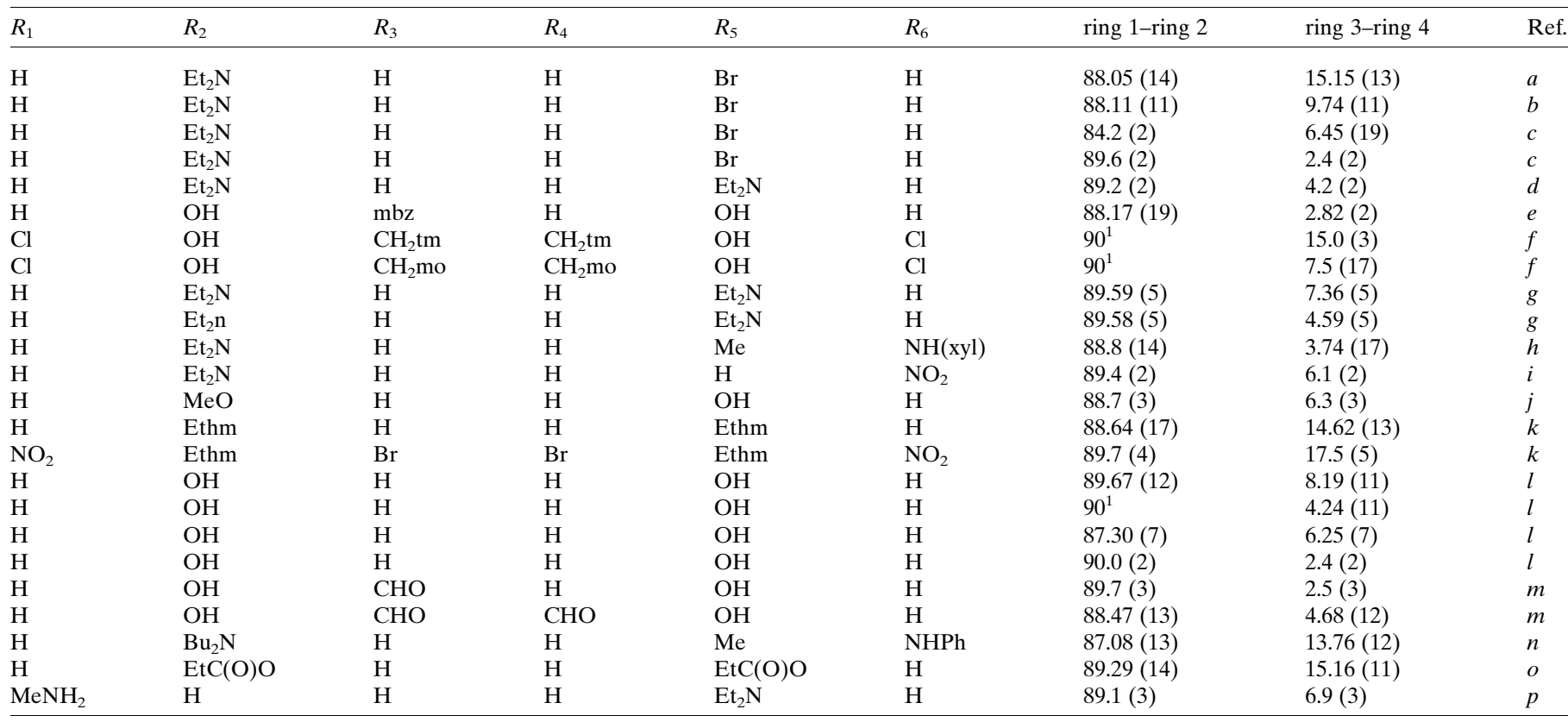

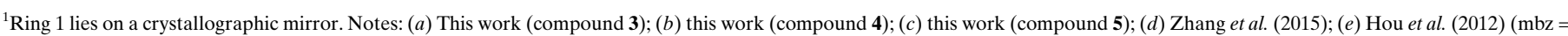

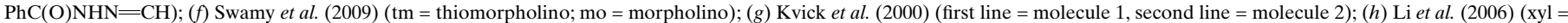

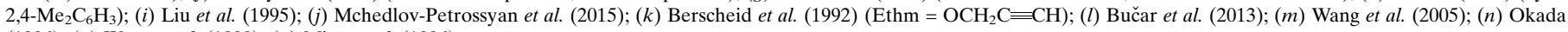
(1996); (o) Wang et al. (1990); (p) Miao et al. (1996).

\section{Structural commentary}

In general terms, the structures of $\mathbf{3 - 5}$ are similar to those of other rhodamine derivatives that have been reported in that the xanthene portion is modestly folded along the $\mathrm{O} \cdots \mathrm{C}$ axis of the central ring and the benzofuranone unit is nearly perpendicular to the mean plane of the xanthene unit. Of note in the present work is the variation in the fold of the xanthene portion which is largest in $\mathbf{3}$, distinctly smaller in $\mathbf{4}$ and smallest in 5 but with a significant difference in this angle between the two independent molecules (see the first four entries in Table 1Fig. 1). We attribute these differences to the different

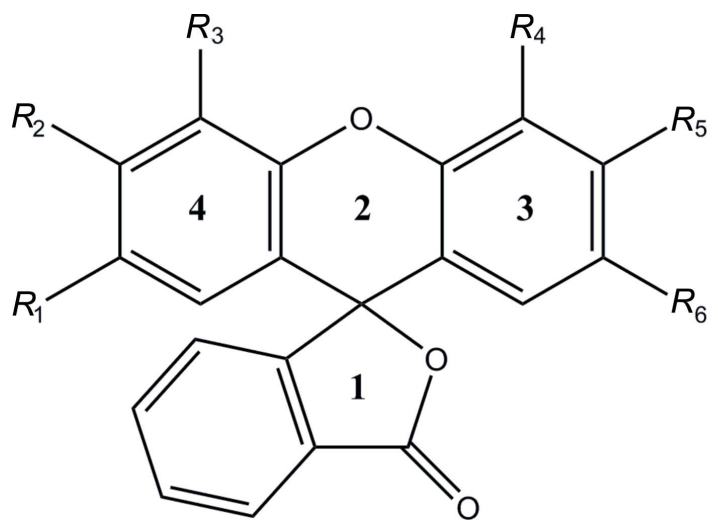

Figure 1

Key for Table 1. packing modes for the three structures. In 3 (Fig. 2), the molecules form zigzag stacks with each pair of adjacent molecules related by centers of symmetry. This leads to pairwise H17C …C4 separations of $3.04 \AA$ which are only $0.14 \AA$ less than the sum of the van der Waals radii. Were the xanthene portions flatter, these would develop into significant intermolecular contacts. With 4 and 5 (Figs. 3 and 4 ) in the noncentrosymmetric space group $P 2_{1} 2_{1} 2_{1}$, this stacking is no longer possible and while in $\mathbf{4}$ there is a van der Waals contact

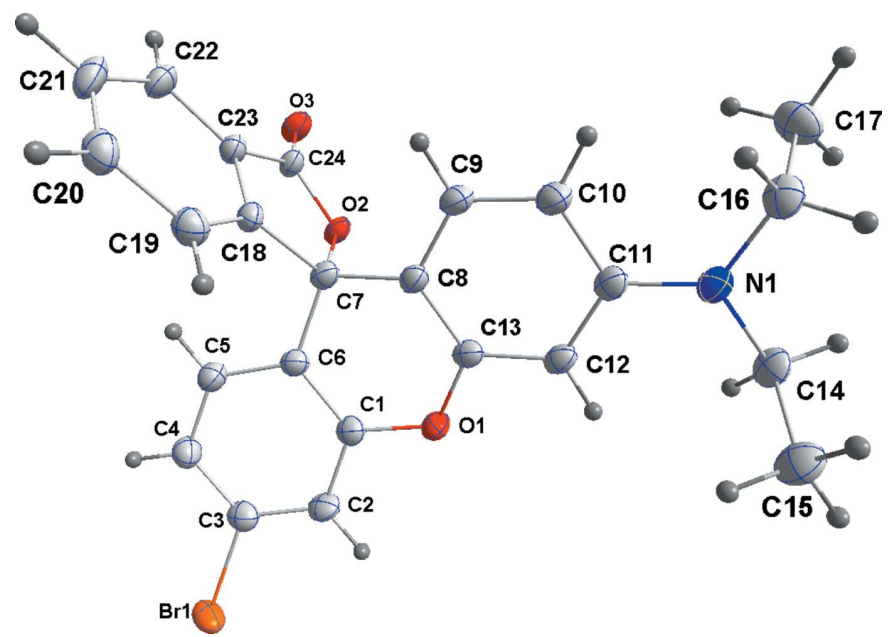

Figure 2

Perspective view of $\mathbf{3}$, with the atom-numbering scheme and $50 \%$ probability displacement ellipsoids. 


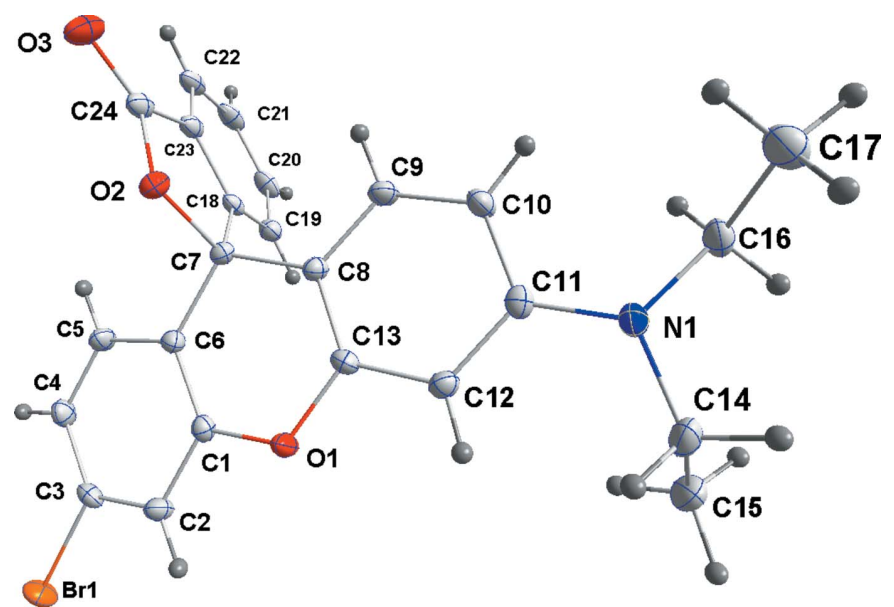

Figure 3

Perspective view of $\mathbf{4}$, with the atom-numbering scheme and $50 \%$ probability displacement ellipsoids.

of $2.90 \AA$ between $\mathrm{H} 17 A$ and $\mathrm{C} 4{ }^{\mathrm{i}}$ [symmetry code: (i) $\frac{3}{2}-x$, $\left.1-y,-\frac{1}{2}+z\right]$ which could be lessened by a greater folding, this is opposed by a $\mathrm{H} 2 \cdots \mathrm{H} 19^{\mathrm{ii}}$ [symmetry code: (ii) $-\frac{1}{2}+x, \frac{3}{2}-y$, $1-z$ ] separation of 2.48 (4) $\AA$ which is only $0.08 \AA$ greater than the sum of the van der Waals radii. In the case of $\mathbf{5}$, the C8-C13 ring experiences the opposing contacts $\mathrm{H} 40 B \cdots \mathrm{C} 13$ $(2.79 \AA)$ and $\mathrm{H} 41 B^{\mathrm{iii}} \ldots \mathrm{C} 11$ [2.79 $\AA$; symmetry code: (iii) $1+x$, $y, z]$, both of which are $0.11 \AA$ less than a van der Waals contact and serve to hold this ring in position in the packing. On the other side of this xanthene moiety there is a $\mathrm{Br} 1 \cdots \mathrm{O}^{\text {iv }}$ [symmetry code: (iv) $\frac{1}{2}+x, \frac{3}{2}-y, 1-z$ ] contact of 3.251 (3) $\AA$ which is $0.12 \AA$ less than a van der Waals contact and imparts more of a twist than a simple fold to this portion. This can be seen from the dihedral angle of $5.7(2)^{\circ}$ between the C1-C6 ring and the $\mathrm{C} 1 / \mathrm{C} 6 / \mathrm{C} 7 / \mathrm{O} 1$ plane. For the second molecule, there are no short intermolecular contacts with either side of the xanthene moiety to influence its conformation.
Table 2

Hydrogen-bond geometry $\left(\AA,^{\circ}\right)$ for $\mathbf{3}$.

$\mathrm{Cg}$ is the centroid of the $\mathrm{C} 8-\mathrm{C} 13$ ring.

\begin{tabular}{lllll}
\hline$D-\mathrm{H} \cdots A$ & $D-\mathrm{H}$ & $\mathrm{H} \cdots A$ & $D \cdots A$ & $D-\mathrm{H} \cdots A$ \\
\hline $\mathrm{C} 14-\mathrm{H} 14 B \cdots \mathrm{O} 2^{\mathrm{i}}$ & 0.99 & 2.68 & $3.649(4)$ & 165 \\
$\mathrm{C}^{\mathrm{i}} 16-\mathrm{H} 16 A \cdots \mathrm{O} 3^{\mathrm{ii}}$ & 0.99 & 2.64 & $3.522(3)$ & 148 \\
$\mathrm{C} 16-\mathrm{H} 16 B \cdots \mathrm{Br}^{\mathrm{iii}}$ & 0.99 & 2.99 & $3.939(3)$ & 162 \\
$\mathrm{C} 17-\mathrm{H} 17 A \cdots \mathrm{Cg}^{\mathrm{iv}}$ & 0.98 & 2.75 & $3.666(4)$ & 156 \\
$\mathrm{C} 19-\mathrm{H} 19 \cdots 1^{\mathrm{iii}}$ & 0.95 & 2.57 & $3.485(4)$ & 161 \\
$\mathrm{C} 20-\mathrm{H} 20 \cdots \mathrm{O}^{\mathrm{v}}$ & 0.95 & 2.58 & $3.421(3)$ & 148 \\
\hline
\end{tabular}

Symmetry codes: (i) $-x,-y+1,-z+1$; (ii) $x, y, z-1$; (iii) $-x+1,-y+1,-z+1$; (iv) $-x+1,-y+2,-z+1$; (v) $x+1, y, z$.

Table 3

Hydrogen-bond geometry $\left(\AA{ }^{\circ}\right)$ for 4.

\begin{tabular}{lllll}
\hline$D-\mathrm{H} \cdots A$ & $D-\mathrm{H}$ & $\mathrm{H} \cdots A$ & $D \cdots A$ & $D-\mathrm{H} \cdots A$ \\
\hline $\mathrm{C} 14-\mathrm{H} 14 B \cdots \mathrm{O}^{\mathrm{i}}$ & $0.99(2)$ & $2.68(2)$ & $3.621(3)$ & $160.7(19)$ \\
$\mathrm{C} 20-\mathrm{H} 20 \cdots 3^{\mathrm{ii}}$ & $0.94(2)$ & $2.41(3)$ & $3.163(3)$ & $138(3)$ \\
\hline
\end{tabular}

Symmetry codes: (i) $-x+2, y+\frac{1}{2},-z+\frac{1}{2}$; (ii) $-x+2, y+\frac{1}{2},-z+\frac{3}{2}$.

\section{Supramolecular features}

Fig. 5 illustrates the intermolecular interactions in the crystal of $\mathbf{3}$ with numerical details given in Table 2. These include two sets of pairwise $\mathrm{C}-\mathrm{H} \cdots \mathrm{O}$ hydrogen bonds, two additional sets of $\mathrm{C}-\mathrm{H} \cdots \mathrm{O}$ hydrogen bonds and a set of $\mathrm{C}-\mathrm{H} \cdots \pi($ ring $)$ interactions. The $\mathrm{C} 14 \cdots \mathrm{H} 14 B \cdots \mathrm{O} 2^{\mathrm{i}}$ and $\mathrm{C} 19-\mathrm{H} 19 \cdots \mathrm{O} 1^{\mathrm{iii}}$ interactions bind the molecules into stacks along the $a$-axis direction while the $\mathrm{C} 16-\mathrm{H} 16 A \cdots \mathrm{O} 3^{\mathrm{i}}$ and $\mathrm{C} 17-$ $\mathrm{H} 17 A \cdots \pi(\text { ring })^{\text {iv }}$ interactions tie the stacks together (Fig. 6). Intermolecular interactions are much fewer in the crystal of 4 with $\mathrm{C} 14-\mathrm{H} 14 B \cdots \mathrm{O}^{\mathrm{v}}$ and $\mathrm{C} 20-\mathrm{H} 20 \cdots \mathrm{O}^{\mathrm{vi}}$ hydrogen bonds (Table 3) forming zigzag chains (Fig. 7) running approximately along the $c$-axis direction and arranged to form rectangular channels along the $a$-axis direction (Fig. 8). In the

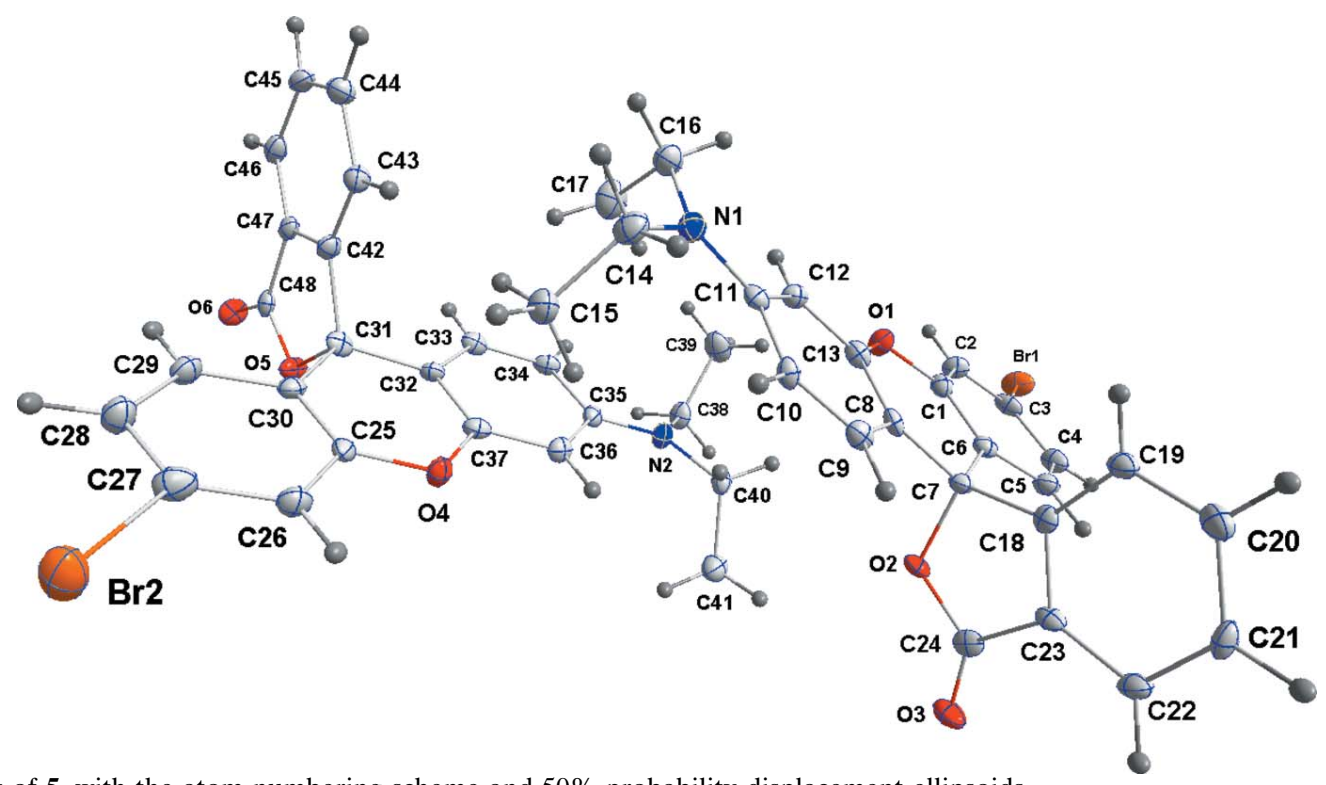

Figure 4

The asymmetric unit of $\mathbf{5}$, with the atom-numbering scheme and $50 \%$ probability displacement ellipsoids. 


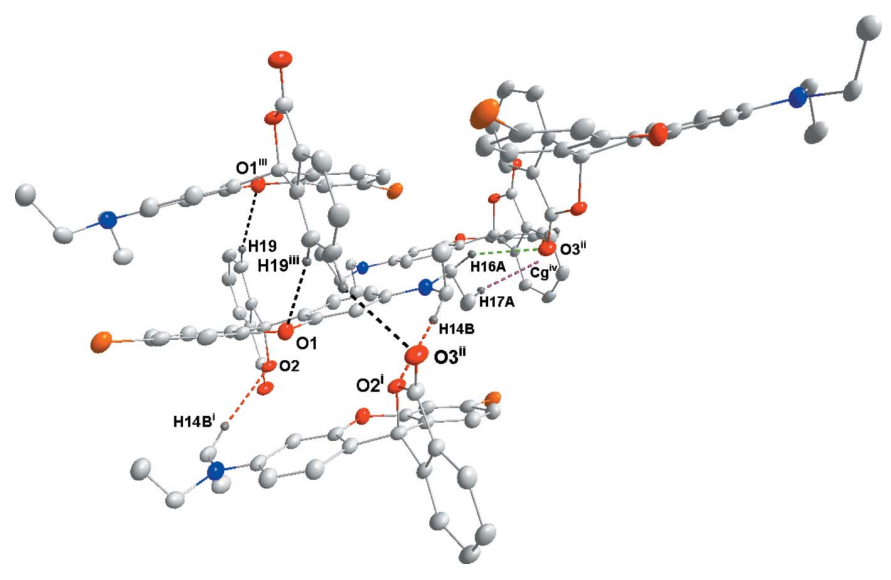

Figure 5

Detail of the intermolecular interactions in $\mathbf{3}$ with $\mathrm{C} 19-\mathrm{H} 19 \ldots \mathrm{O} 1^{\mathrm{iii}}$, $\mathrm{C} 14-\mathrm{H} 14 B \cdots \mathrm{O} 2^{\mathrm{i}}$, and $\mathrm{C} 16-\mathrm{H} 16 A \cdots \mathrm{O} 3^{\mathrm{ii}}$ hydrogen bonds shown, respectively, as black, red and green dotted lines, while the $\mathrm{C} 17-$ $\mathrm{H} 17 A \cdots C g^{\text {iv }}$ interaction is given by a purple dotted line. [Symmetry codes: (i) $x, y,-1+z$; (ii) $-x, 1-y, 1-z$; (iii) $1-x, 1-y, 1-z$; (iv) $1-x, 2-y, 1-z ; C g$ is the centroid of the indicated ring.]

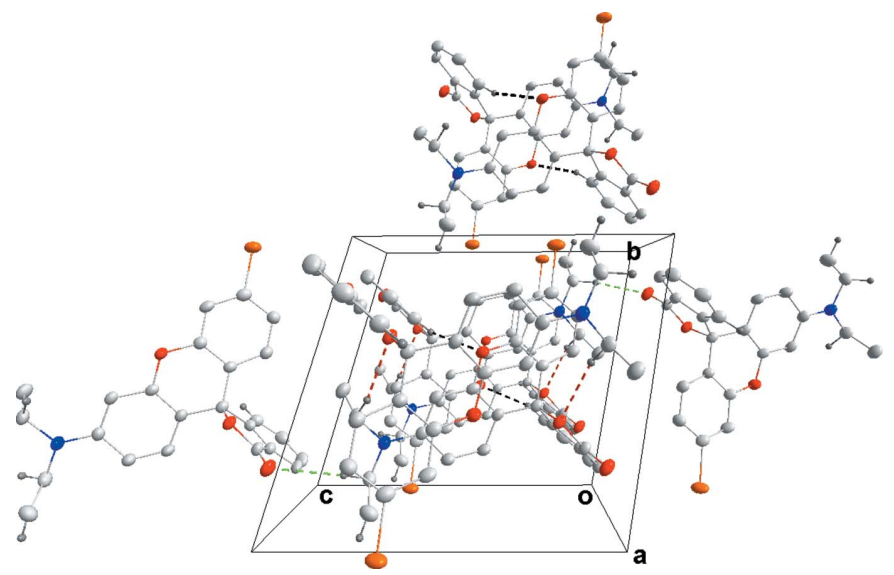

Figure 6

Packing of $\mathbf{3}$, viewed along the $a$-axis direction, with the color code for $\mathrm{C}-\mathrm{H} \cdots \mathrm{O}$ interactions as in Fig. 5.

crystal of $\mathbf{5}$, the two independent molecules are associated through $\mathrm{C} 40-\mathrm{H} 40 \mathrm{~A} \cdots \mathrm{Cg} 1$ and $\mathrm{C} 40-\mathrm{H} 40 \mathrm{~B} \cdots \mathrm{Cg} 2$ interactions with these units tied together on one side by $\mathrm{C} 16-$ $\mathrm{H} 16 B \cdots C g^{\mathrm{i}}$ interactions (Table 4 ) and on the other by a $\pi-\pi$

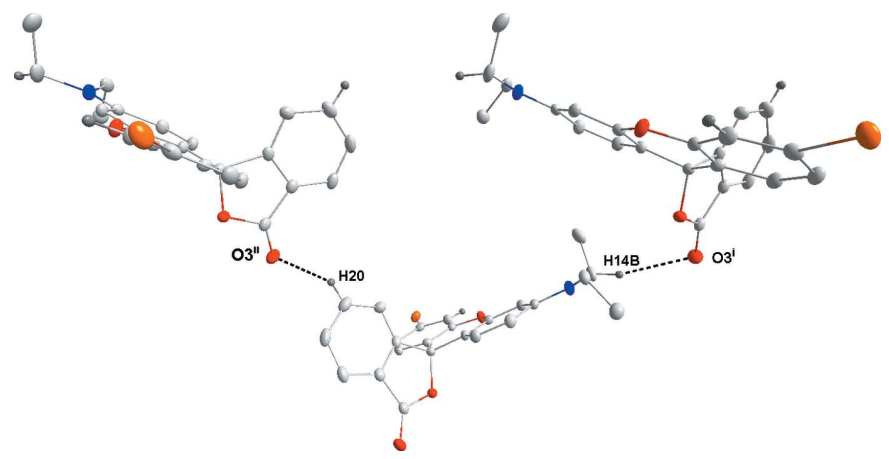

Figure 7

Detail of the intermolecular interactions in 4. [Symmetry codes: (v) $2-x$, $\frac{1}{2}+y, \frac{1}{2}-z ;(\mathrm{vi}) 2-x, \frac{1}{2}+y, \frac{3}{2}-z$.]
Table 4

Hydrogen-bond geometry $\left(\AA,^{\circ}\right)$ for $\mathbf{5}$.

$\mathrm{Cg} 1$ and $\mathrm{Cg} 2$ are the centroids of the $\mathrm{C} 8-\mathrm{C} 13$ and $\mathrm{O} 1, \mathrm{C} 1, \mathrm{C} 6, \mathrm{C} 7, \mathrm{C} 8, \mathrm{C} 13$ rings, respectively.

\begin{tabular}{lllll}
\hline$D-\mathrm{H} \cdots A$ & $D-\mathrm{H}$ & $\mathrm{H} \cdots A$ & $D \cdots A$ & $D-\mathrm{H} \cdots A$ \\
\hline $\mathrm{C} 16-\mathrm{H} 16 B \cdots C g^{\mathrm{i}}$ & 0.99 & 2.81 & $3.583(4)$ & 136 \\
$\mathrm{C} 40-\mathrm{H} 40 A \cdots C g 1$ & 0.99 & 2.79 & $3.534(4)$ & 132 \\
$\mathrm{C} 40-\mathrm{H} 40 B \cdots C g 2$ & 0.99 & 2.83 & $3.580(4)$ & 133 \\
\hline
\end{tabular}

Symmetry code: (i) $x+1, y, z$.

interaction between the $\mathrm{C} 24=\mathrm{O} 3$ bond and the $(\mathrm{C} 18-\mathrm{C} 23)^{\mathrm{ii}}$ ring [Fig. 9, centroid-centroid distance $=3.349$ (3) $\AA$, angle of $\mathrm{C}=\mathrm{O}$ vector to centroid $=99.5(3)^{\circ}$. The result is a more open 3D structure for this enantiomer (Fig. 10).

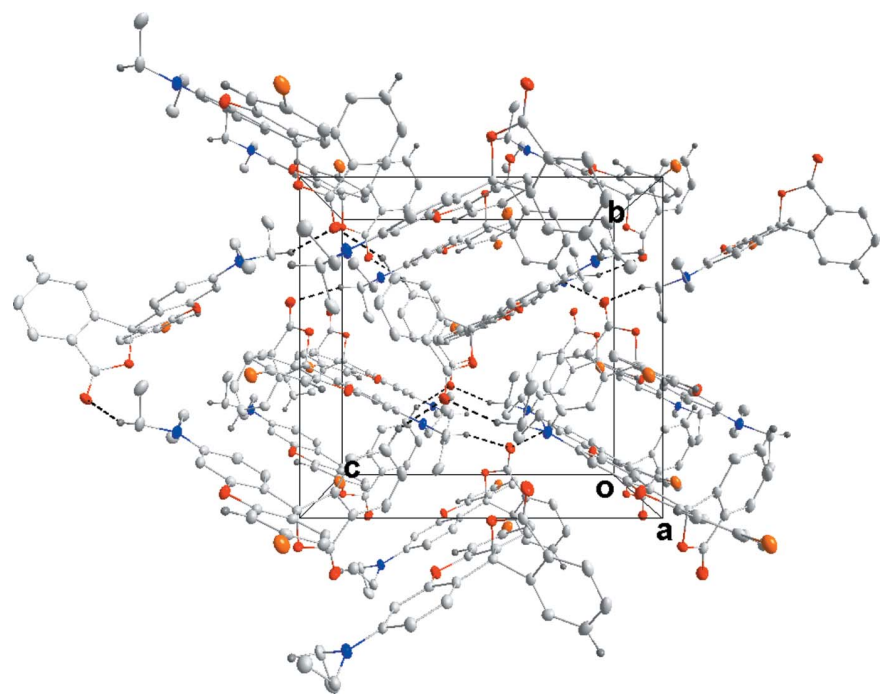

Figure 8

Packing of 4, viewed along the $a$-axis direction, with $\mathrm{C}-\mathrm{H} \cdots \mathrm{O}$ hydrogen bonds shown as dotted lines.

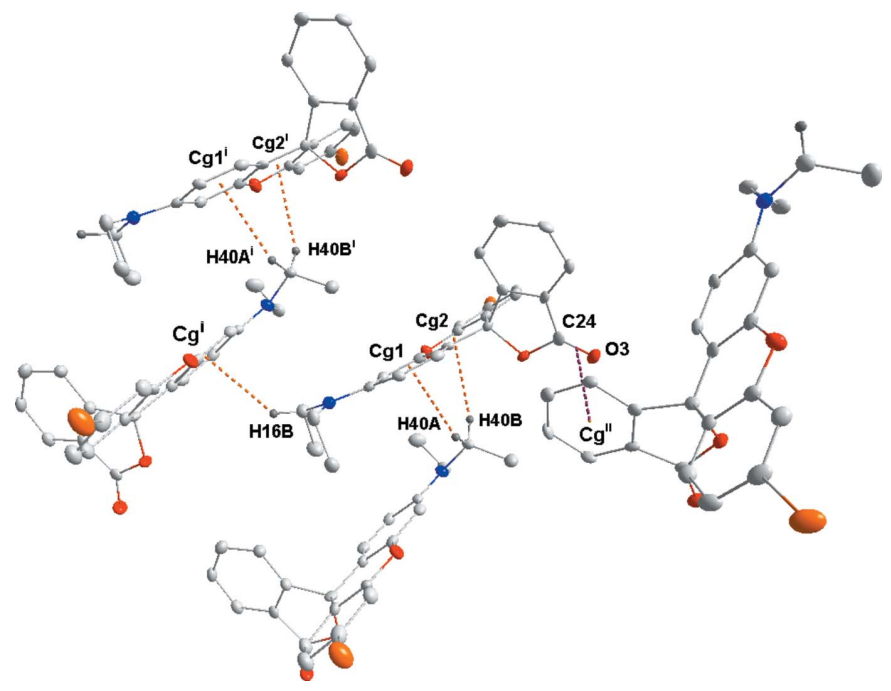

Figure 9

Detail of the intermolecular interactions in $\mathbf{5}$. [Symmetry codes: (vii) $1+x$, $y, z ;($ viii $\left.)-\frac{1}{2}+x, \frac{1}{2}-y, 1-z.\right]$ 


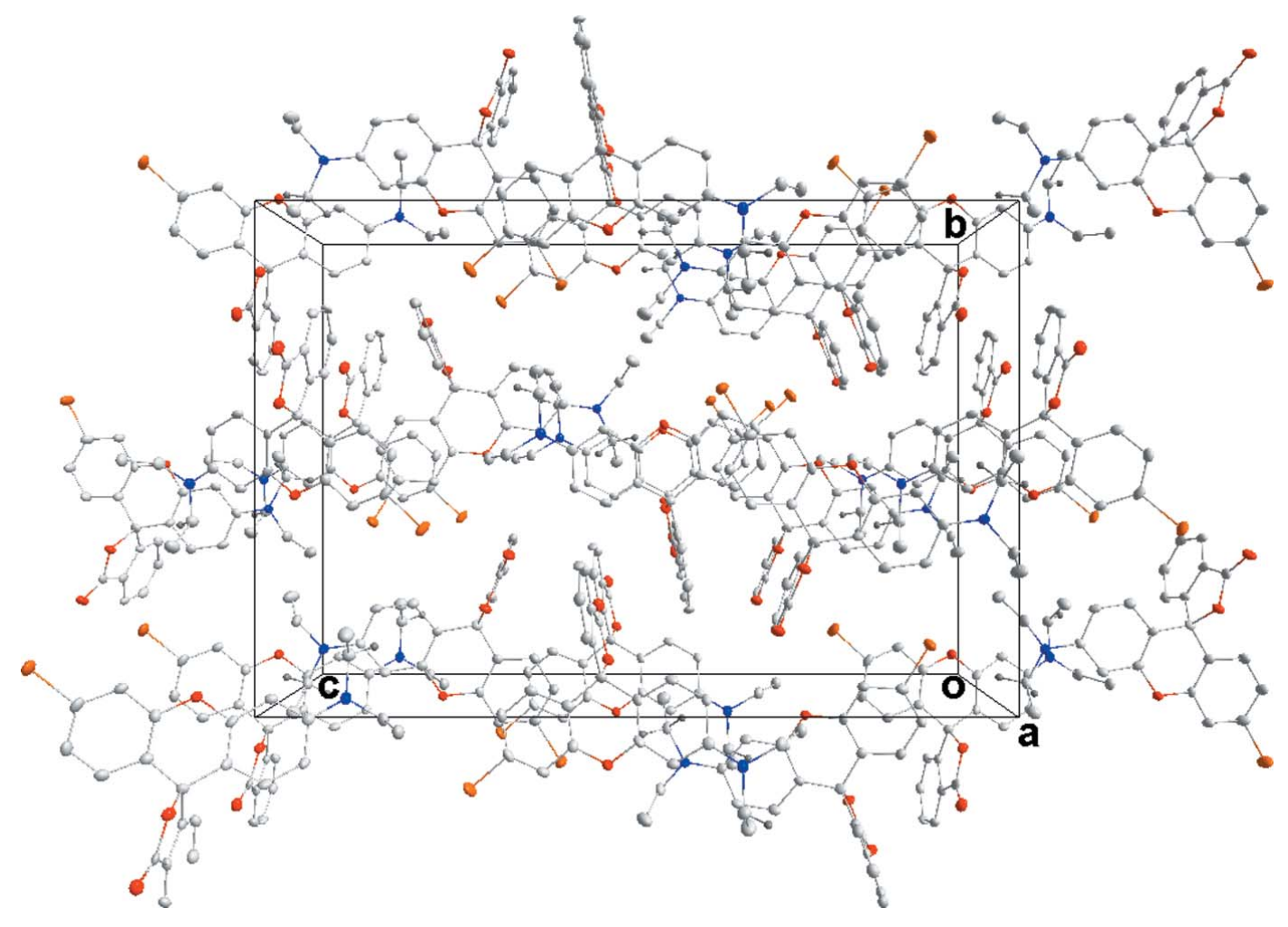

Figure 10

Packing of $\mathbf{5}$, viewed along the $a$-axis direction.

\section{Database survey}

There are 71 structures of rhodamine derivatives in the literature, although many are considerably more substituted than $\mathbf{4}$ and $\mathbf{5}$ and include a variety of fused-ring systems. Table 1 lists, in addition to those reported here, 20 other structures which are most nearly comparable to those of this work. In all of these, the lactone ring (ring 1, Fig. 1) is nearly perpendicular to the mean plane of the central pyran ring (ring 2, Fig. 1) with dihedral angles ranging from 87.08 (13) to $90.0(2)^{\circ}$ and with three structures having the lactone ring on a crystallographic mirror (Table 1). In all cases, the xanthene moiety is folded across the $\mathrm{O} \cdots \mathrm{C}$ axis, with the majority having a dihedral angle between rings 3 and 4 (Fig. 1) in the range $2.42(3)-7.36(5)^{\circ}$, but there are six having angles up to $17.5(5)^{\circ}$ (Table 1). In this latter group, those with the largest angles involve a twist of the xanthene moiety as well as a fold, and this is seen in both symmetrically and unsymmetrically substituted examples. Inspection of intermolecular contact calculations indicates that the largest dihedral angles correlate with intermolecular contacts at or somewhat less than the sums of the relevant van der Waals radii.

\section{Synthesis and crystallization}

As outlined in the scheme, compound $1(2.00 \mathrm{~g}, 6.73 \mathrm{mmol})$ was mixed with compound $2(1.10 \mathrm{~g}, 6.35 \mathrm{mmol})$ in $16 \mathrm{~mL}$ of methylsulfonic acid. The mixture was stirred and heated for $1 \mathrm{~h}$ at $373 \mathrm{~K}$. The cooled solution was poured over ice and then extracted with dicholoromethane. A crude yield of the race- mate 3 was obtained. A portion of the crude product (1.343 g) was purified on a flash column with $15 \%$ ethyl acetate in hexanes followed by $100 \%$ ethyl acetate. The fractions containing the product were combined and left in a beaker covered with a tissue and the solvent was allowed to evaporate slowly. After about two weeks, the purified racemate yielded a mixture of long needle-shaped as well as plate-shaped crystals $(0.293 \mathrm{~g}, 0.651 \mathrm{mmol}, 21.8 \%$ yield $)$. Thin layer chromatography demonstrated that both crystal shapes were the desired product (racemate $\mathbf{3}$ ), but only the needles provided a wellrefined structure. The melting point range was found to be $420.6-428.9 \mathrm{~K}$ for the needles and $415.9-429.8 \mathrm{~K}$ for the plates. An NMR spectrum of compound $\mathbf{3}$ was also obtained (Figs. S1 and S2).

To separate the enantiomers a mobile phase of $70 \%$ hexanes, $29.97 \%$ ethanol and $0.03 \%$ diethylamine was used. A $4 \mathrm{mg} \mathrm{mL}^{-1}$ solution of the racemic bromo-rhodamine derivative, $\mathbf{3}$ was dissolved in the mobile phase. A two-pump system, both Shimadzu LC-20AD pumps, was utilized for moving the mobile phase through the column. Pump A pumped hexanes and Pump B pumped the mixture of $95 \%$ ethanol and $0.5 \%$ diethylamine at a flow rate of $3.0 \mathrm{~mL} \mathrm{~min}^{-1}$ for a total of 16 minutes. The sample was placed in a Shimadzu SIL-20AC autosampler, which injected $400 \mu \mathrm{L}$ of the sample into the mobile phase. A Shimadzu CTO-20A oven, set at 298 $\mathrm{k}$, held the ChiralPak AD-H column whose stationary phase is amylose tris (3,5-dimethylphenylcarbamate) coated on $5 \mu \mathrm{m}$ silica-gel. The compounds were eluted and then detected with a Shimadzu SPD-20A UV photodiode array detector with a deuterium lamp set at $230 \mathrm{~nm}$. Each enantiomer was collected 
Table 5

Experimental details.

3

Crystal data

Chemical formula

$M_{\mathrm{r}}$

Crystal system, space group

Temperature $(\mathrm{K})$

$a, b, c(\AA)$

$\alpha, \beta, \gamma\left(^{\circ}\right)$

$V\left(\AA^{3}\right)$

Z

Radiation type

$\mu\left(\mathrm{mm}^{-1}\right)$

Crystal size (mm)

Data collection

Diffractometer

Absorption correction

$T_{\min }, T_{\max }$

No. of measured, independent and

observed $[I>2 \sigma(I)]$ reflections

$R_{\text {int }}$

$(\sin \theta / \lambda)_{\max }\left(\AA^{-1}\right)$

Refinement

$R\left[F^{2}>2 \sigma\left(F^{2}\right)\right], w R\left(F^{2}\right), S$

No. of reflections

No. of parameters

$\mathrm{H}$-atom treatment

$\Delta \rho_{\max }, \Delta \rho_{\min }\left(\mathrm{e} \AA^{-3}\right)$

Absolute structure

Absolute structure parameter

$-$
4

$\begin{array}{ll}\mathrm{C}_{24} \mathrm{H}_{20} \mathrm{BrNO}_{3} & \mathrm{C}_{24} \mathrm{H}_{20} \mathrm{BrNO}_{3} \\ 450.32 & 450.32 \\ \text { Triclinic, } P \overline{1} & \text { Orthorhombic, } P 2_{1} 2_{1} 2_{1} \\ 150 & 100 \\ 8.3074(4), 11.1871(5), 11.7693(6) & 11.0772(6), 13.0582(8) \\ & 13.8966(8) \\ 102.384(2), 91.106(2), 109.581(2) & 90,90,90 \\ 1001.60(8) & 2010.1(2) \\ 2 & 4 \\ \mathrm{Cu} K \alpha & \mathrm{Mo} \mathrm{K \alpha} \\ 3.01 & 2.07 \\ 0.11 \times 0.07 \times 0.06 & 0.31 \times 0.12 \times 0.10\end{array}$

5

Bruker D8 VENTURE PHOTON 100 CMOS

Multi-scan ( $S A D A B S$; Bruker, 2016)

$0.59,0.84$

$7525,3725,3415$

0.037

0.618

$0.045,0.124,1.03$

3725

264

$\mathrm{H}$-atom parameters constrained

$1.01,-0.98$
Bruker SMART APEX CCD

Multi-scan ( $S A D A B S$; Bruker, 2016)

$0.69,0.82$

$39006,5417,4926$

0.043

0.687

$0.026,0.058,0.99$

5417

320

$\mathrm{H}$ atoms treated by a mixture of independent and constrained refinement

$0.62,-0.25$

Flack $x$ determined using 1981 quotients $\left[\left(I^{+}\right)-\left(I^{-}\right)\right] /\left[\left(I^{+}\right)+\left(I^{-}\right)\right.$ (Parsons et al., 2013)

-0.006 (3)

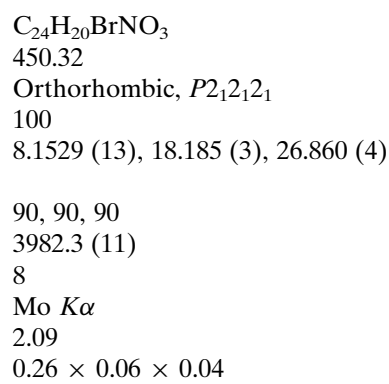

Bruker SMART APEX CCD

Multi-scan (SADABS; Bruker, 2016)

$0.70,0.92$

38240, 10174, 7285

0.075

0.685

$0.045,0.093,0.97$

10174

527

$\mathrm{H}$-atom parameters constrained

$0.92,-0.34$

Flack $x$ determined using 2575 quotients $\left[\left(I^{+}\right)-\left(I^{-}\right)\right] /\left[\left(I^{+}\right)+\left(I^{-}\right)\right.$ (Parsons et al., 2013)

-0.002 (6)

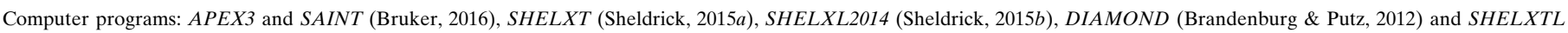
(Sheldrick, 2008).

with a Shimadzu FRC-10A fraction collector. One enantiomer (4) elutes from 11.6-12.8 minutes, and the other (5) elutes from 13.4-14.8 minutes using the method described above. Slow evaporation of the solutions of the pure enantiomers at room temperature afforded X-ray quality crystals over 1-5 days.

\section{Refinement}

Crystal data, data collection and structure refinement details are summarized in Table 5. In $\mathbf{3}$ and $\mathbf{5}, \mathbf{H}$ atoms attached to carbon were placed in calculated positions $(\mathrm{C}-\mathrm{H}=0.95-$ $0.99 \AA$ ) and included as riding contributions with isotropic displacement parameters 1.2-1.5 times those of the attached atoms. In 4, the methyl group $\mathrm{H}$ atoms were placed in calculated positions as in $\mathbf{3}$ and $\mathbf{5}$ (due to poor geometry resulting from individual refinement) while the remainder were refined.

\section{Acknowledgements}

The support of NSF-MRI Grant No. 1228232 for the purchase of the D8 diffractometer and Tulane University for support of the Tulane Crystallography Laboratory are gratefully acknowledged.

\section{References}

Berscheid, R., Nieger, M. \& Vögtle, F. (1992). Chem. Ber. 125, 2539 2552.

Brandenburg, K. \& Putz, H. (2012). DIAMOND. Crystal Impact GbR, Bonn, Germany.

Bruker (2016). APEX3, SADABS and SAINT. Bruker AXS Inc., Madison, Wisconsin, USA.

Bučar, D.-K., Filip, S., Arhangelskis, M., Lloyd, G. O. \& Jones, W. (2013). CrystEngComm, 15, 6289-6291.

Chen, X. Q., Pradhan, T., Wang, F., Kim, J. S. \& Yoon, J. (2012). Chem. Rev. 112, 1910-1956.

Hou, F., Cheng, J., Xi, P., Chen, F., Huang, L., Xie, G., Shi, Y., Liu, H., Bai, D. \& Zeng, Z. (2012). Dalton Trans. 41, 5799-5804.

Jo, H. H., Lin, C. Y. \& Anslyn, E. V. (2014). Acc. Chem. Res. 47, 22122221.

Kvick, Å., Vaughan, G. B. M., Wang, X., Sun, Y. \& Long, Y. (2000). Acta Cryst. C56, 1232-1233

LaPlante, S. R. F., Fader, L. D., Fandrick, K. R., Fandrick, D. R., Hucke, O., Kemper, R., Miller, S. P. F. \& Edwards, P. J. (2011). J. Med. Chem. 54, 7005-7022. 
Li, X. M., Ding, C. F., Tian, B. Q., Liu, Q., Zhang, S. S., Xu, H. \& Ouyang, P. K. (2006). Chem. Pap. 60, 220-223.

Liu, X.-L., Wang, J.-L., Liu, J.-W. \& Miao, F.-M. (1995). Acta Cryst. C51, 324-326.

Mchedlov-Petrossyan, N. O., Cheipesh, T. A., Shekhovtsov, S. V., Redko, A. N., Rybachenko, V. I., Omelchenko, I. V. \& Shishkin, O. V. (2015). Spectrochim. Acta Part A, 150, 151-161.

Miao, F.-M., Zhang, L.-J., Wen, X., Zhou, W.-H., Niu, Z.-C., Han, J.-G. \& Liu, X.-L. (1996). Acta Cryst. C52, 700-702.

Okada, K. (1996). J. Mol. Struct. 380, 235-247.

Pak, Y. L., Swamy, K. M. \& Yoon, J. (2015). Sensors (Basel), 15, 24374-24396.

Parsons, S., Flack, H. D. \& Wagner, T. (2013). Acta Cryst. B69, 249259.

Reist, M., Carrupt, P. A., Francotte, E. \& Testa, B. (1998). Chem. Res. Toxicol. 11, 1521-1528.

Sheldrick, G. M. (2008). Acta Cryst. A64, 112-122.

Sheldrick, G. M. (2015a). Acta Cryst. A71, 3-8.
Sheldrick, G. M. (2015b). Acta Cryst. C71, 3-8.

Shimizu, K. D. \& Stephenson, C. J. (2010). Curr. Opin. Chem. Biol. 14, 743-750.

Swamy, K. M. K., Kim, H. N., Soh, J. H., Kim, Y., Kim, S.-J. \& Yoon, J. (2009). Chem. Commun. pp. 1234-1236.

Wang, M., Marriott, P. J., Chan, W. H., Lee, A. W. M. \& Huie, C. W. (2006). J. Chromatogr. A, 1112, 361-368.

Wang, W., Rusin, O., Xu, X., Kim, K. K., Escobedo, J. O., Fakayode, S. O., Fletcher, K. A., Lowry, M., Schowalter, C. M., Lawrence, C. M., Fronczek, F. R., Warner, I. M. \& Strongin, R. M. (2005). J. Am. Chem. Soc. 127, 15949-15958.

Wang, L.-F., Wang, X., Peng, Z., He, F. \& Wang, Q. (1990). Acta Cryst. C46, 1676-1678.

You, L., Zha, D. \& Anslyn, E. V. (2015). Chem. Rev. 115, 7840-7892.

Yu, S. \& Pu, L. (2015). Tetrahedron, 71, 745-772.

Zhang, I., Wang, Y., Wan, C., Xing, Z., Li, W., Li, M. \& Zhang, S. X.-A. (2015). RSC Adv. 5, 66416-66419.

Zhang, X., Yin, J. \& Yoon, J. (2014). Chem. Rev. 114, 4918-4959. 


\section{supporting information}

Acta Cryst. (2017). E73, 327-333 [https://doi.org/10.1107/S2056989017001451]

Synthesis, resolution and crystal structures of two enantiomeric rhodamine derivatives

Clifton J. Stephenson, Joel T. Mague, Nathaniel Kamm, Nathalie Aleman, Dayla Rich, QuynhNhu Dang and Ha Van Nguyen

Computing details

For all compounds, data collection: APEX3 (Bruker, 2016); cell refinement: SAINT (Bruker, 2016); data reduction: SAINT (Bruker, 2016); program(s) used to solve structure: SHELXT (Sheldrick, 2015a); program(s) used to refine structure: SHELXL2014 (Sheldrick, 2015b); molecular graphics: DIAMOND (Brandenburg \& Putz, 2012); software used to prepare material for publication: SHELXTL (Sheldrick, 2008).

(3) rac-6'-Bromo-3'-diethylamino-3H-spiro[2-benzofuran-1,9'-xanthen]-3-one

Crystal data

$\mathrm{C}_{24} \mathrm{H}_{20} \mathrm{BrNO}_{3}$

$M_{r}=450.32$

Triclinic, $P \overline{1}$

$a=8.3074(4) \AA$

$b=11.1871(5) \AA$

$c=11.7693(6) \AA$

$\alpha=102.384(2)^{\circ}$

$\beta=91.106(2)^{\circ}$

$\gamma=109.581(2)^{\circ}$

$V=1001.60(8) \AA^{3}$

Data collection

Bruker D8 VENTURE PHOTON 100 CMOS diffractometer

Radiation source: INCOATEC I $\mu$ S micro-focus source

Mirror monochromator

Detector resolution: 10.4167 pixels $\mathrm{mm}^{-1}$

$\omega$ scans

Absorption correction: multi-scan

(SADABS; Bruker, 2016)

Refinement

Refinement on $F^{2}$

Least-squares matrix: full

$R\left[F^{2}>2 \sigma\left(F^{2}\right)\right]=0.045$

$w R\left(F^{2}\right)=0.124$

$S=1.03$

3725 reflections
$Z=2$

$F(000)=460$

$D_{\mathrm{x}}=1.493 \mathrm{Mg} \mathrm{m}^{-3}$

$\mathrm{Cu} K \alpha$ radiation, $\lambda=1.54178 \AA$

Cell parameters from 6586 reflections

$\theta=3.9-72.5^{\circ}$

$\mu=3.01 \mathrm{~mm}^{-1}$

$T=150 \mathrm{~K}$

Column, colourless

$0.11 \times 0.07 \times 0.06 \mathrm{~mm}$

$T_{\min }=0.59, T_{\max }=0.84$

7525 measured reflections

3725 independent reflections

3415 reflections with $I>2 \sigma(I)$

$R_{\text {int }}=0.037$

$\theta_{\max }=72.5^{\circ}, \theta_{\min }=3.9^{\circ}$

$h=-10 \rightarrow 9$

$k=-13 \rightarrow 13$

$l=-12 \rightarrow 14$

264 parameters

0 restraints

Primary atom site location: structure-invariant direct methods

Secondary atom site location: difference Fourier map 
Hydrogen site location: inferred from neighbouring sites

H-atom parameters constrained

$$
\begin{aligned}
& w=1 /\left[\sigma^{2}\left(F_{\mathrm{o}}^{2}\right)+(0.0814 P)^{2}+0.6226 P\right] \\
& \text { where } P=\left(F_{\mathrm{o}}^{2}+2 F_{\mathrm{c}}^{2}\right) / 3 \\
& (\Delta / \sigma)_{\max }<0.001 \\
& \Delta \rho_{\max }=1.01 \text { e } \AA^{-3} \\
& \Delta \rho_{\min }=-0.98 \text { e } \AA^{-3}
\end{aligned}
$$

Special details

Geometry. All esds (except the esd in the dihedral angle between two 1.s. planes) are estimated using the full covariance matrix. The cell esds are taken into account individually in the estimation of esds in distances, angles and torsion angles; correlations between esds in cell parameters are only used when they are defined by crystal symmetry. An approximate (isotropic) treatment of cell esds is used for estimating esds involving l.s. planes.

Refinement. Refinement of $\mathrm{F}^{2}$ against ALL reflections. The weighted $\mathrm{R}$-factor $\mathrm{wR}$ and goodness of fit $\mathrm{S}$ are based on $\mathrm{F}^{2}$, conventional $\mathrm{R}$-factors $\mathrm{R}$ are based on $\mathrm{F}$, with $\mathrm{F}$ set to zero for negative $\mathrm{F}^{2}$. The threshold expression of $\mathrm{F}^{2}>2$ sigma $\left(\mathrm{F}^{2}\right)$ is used only for calculating R-factors ( $\mathrm{gt}$ ) etc. and is not relevant to the choice of reflections for refinement. R-factors based

\begin{tabular}{|c|c|c|c|c|}
\hline & $x$ & $y$ & $z$ & $U_{\text {iso }} * / U_{\text {eq }}$ \\
\hline Br1 & $0.19033(4)$ & $0.02441(3)$ & $0.64991(3)$ & $0.03278(14)$ \\
\hline $\mathrm{O} 1$ & $0.1667(2)$ & $0.41446(18)$ & $0.49152(16)$ & $0.0248(4)$ \\
\hline $\mathrm{O} 2$ & $0.2042(2)$ & $0.65283(18)$ & $0.77320(15)$ & $0.0217(4)$ \\
\hline $\mathrm{O} 3$ & 0.1991 & $0.7974(2)$ & $0.93699(17)$ & $0.0301(4)$ \\
\hline N1 & $0.2392(3)$ & $0.7234(2)$ & 0.25883 (19) & $0.0265(5)$ \\
\hline $\mathrm{C} 1$ & 0.2155 & $0.3798(3)$ & $0.5878(2)$ & $0.0224(5)$ \\
\hline $\mathrm{C} 2$ & $0.1813(3)$ & $0.2475(3)$ & $0.5752(2)$ & $0.0257(5)$ \\
\hline $\mathrm{H} 2$ & 0.1266 & 0.1871 & 0.5040 & $0.031^{*}$ \\
\hline C3 & $0.2281(3)$ & $0.2048(3)$ & $0.6683(2)$ & $0.0263(6)$ \\
\hline $\mathrm{C} 4$ & $0.3066(4)$ & 0.2908 & $0.7742(2)$ & $0.0272(6)$ \\
\hline $\mathrm{H} 4$ & 0.3360 & 0.2601 & 0.8381 & $0.033^{*}$ \\
\hline $\mathrm{C} 5$ & 0.3404 & 0.4226 & $0.7836(2)$ & $0.0256(5)$ \\
\hline H5 & 0.3948 & 0.4826 & 0.8551 & $0.031 *$ \\
\hline C6 & 0.2970 & $0.4704(3)$ & $0.6915(2)$ & $0.0210(5)$ \\
\hline $\mathrm{C} 7$ & $0.3359(3)$ & 0.6138 & $0.7025(2)$ & $0.0205(5)$ \\
\hline $\mathrm{C} 8$ & $0.3204(3)$ & $0.6420(3)$ & $0.5850(2)$ & $0.0213(5)$ \\
\hline C9 & $0.3837(3)$ & $0.7690(3)$ & $0.5676(2)$ & $0.0256(6)$ \\
\hline $\mathrm{H} 9$ & 0.4463 & 0.8386 & 0.6313 & $0.031 *$ \\
\hline $\mathrm{C} 10$ & 0.3584 & 0.7966 & $0.4618(2)$ & $0.0268(6)$ \\
\hline $\mathrm{H} 10$ & 0.4044 & 0.8841 & 0.4540 & $0.032 *$ \\
\hline $\mathrm{C} 11$ & $0.2647(3)$ & $0.6962(3)$ & $0.3641(2)$ & $0.0228(5)$ \\
\hline $\mathrm{C} 12$ & 0.1999 & 0.5689 & $0.3810(2)$ & $0.0227(5)$ \\
\hline $\mathrm{H} 12$ & 0.1348 & 0.4990 & 0.3182 & $0.027 *$ \\
\hline $\mathrm{C} 13$ & $0.2302(3)$ & $0.5445(2)$ & $0.4882(2)$ & $0.0206(5)$ \\
\hline $\mathrm{C} 14$ & $0.1323(3)$ & $0.6234(3)$ & $0.1601(2)$ & $0.0265(6)$ \\
\hline $\mathrm{H} 14 \mathrm{~A}$ & 0.0819 & 0.6652 & 0.1101 & $0.032 *$ \\
\hline H14B & 0.0366 & 0.5614 & 0.1899 & $0.032 *$ \\
\hline C15 & $0.2312(4)$ & $0.5483(3)$ & $0.0865(3)$ & $0.0342(7)$ \\
\hline H15A & 0.1533 & 0.4819 & 0.0223 & $0.051 *$ \\
\hline
\end{tabular}
on $\mathrm{F}^{2}$ are statistically about twice as large as those based on $\mathrm{F}$, and $\mathrm{R}$ - factors based on ALL data will be even larger. $\mathrm{H}$ atoms attached to carbon were placed in calculated positions $(\mathrm{C}-\mathrm{H}=0.95-0.99 \AA)$ and included as riding contributions with isotropic displacement parameters $1.2-1.5$ times those of the attached atoms.

Fractional atomic coordinates and isotropic or equivalent isotropic displacement parameters $\left(\AA^{2}\right)$ 


$\begin{array}{lllll}\text { H15B } & 0.2806 & 0.5060 & 0.1354 & 0.051^{*} \\ \text { H15C } & 0.3236 & 0.6087 & 0.0545 & 0.051^{*} \\ \text { C16 } & 0.3190(3) & 0.8529(3) & 0.2376(2) & 0.0271(6) \\ \text { H16A } & 0.3361 & 0.8444 & 0.1536 & 0.033^{*} \\ \text { H16B } & 0.4334 & 0.8949 & 0.2827 & 0.033^{*} \\ \text { C17 } & 0.2128(4) & 0.9391(3) & 0.2713(3) & 0.0376(7) \\ \text { H17A } & 0.2746 & 1.0263 & 0.2594 & 0.056^{*} \\ \text { H17B } & 0.1924 & 0.9457 & 0.3538 & 0.056^{*} \\ \text { H17C } & 0.1027 & 0.9013 & 0.2227 & 0.056^{*} \\ \text { C18 } & 0.5013(3) & 0.6985(2) & 0.7778(2) & 0.0205(5) \\ \text { C19 } & 0.6676(3) & 0.7027(3) & 0.7611(3) & 0.0267(6) \\ \text { H19 } & 0.6922 & 0.6516 & 0.6928 & 0.032^{*} \\ \text { C20 } & 0.7980(3) & 0.7847(3) & 0.8483(3) & 0.0306(6) \\ \text { H20 } & 0.9128 & 0.7881 & 0.8399 & 0.037^{*} \\ \text { C21 } & 0.7623(4) & 0.8615(3) & 0.9472(3) & 0.0317(6) \\ \text { H21 } & 0.8530 & 0.9160 & 1.0054 & 0.038^{*} \\ \text { C22 } & 0.5968(4) & 0.8599(3) & 0.9619(2) & 0.0270(6) \\ \text { H22 } & 0.5725 & 0.9142 & 1.0282 & 0.032^{*} \\ \text { C23 } & 0.4670(3) & 0.7756(3) & 0.8758(2) & 0.0208(5) \\ \text { C24 } & 0.2804(3) & 0.7489(3) & 0.8704(2) & 0.0220(5)\end{array}$

Atomic displacement parameters $\left(\AA^{2}\right)$

\begin{tabular}{lllllll}
\hline & $U^{11}$ & $U^{22}$ & $U^{33}$ & $U^{12}$ & $U^{13}$ & $U^{23}$ \\
\hline Br1 & $0.02583(19)$ & $0.02119(19)$ & $0.0485(2)$ & $0.00731(13)$ & $-0.00533(13)$ & $0.00453(13)$ \\
O1 & $0.0247(9)$ & $0.0208(10)$ & $0.0236(9)$ & $0.0047(7)$ & $-0.0068(7)$ & $0.0006(7)$ \\
O2 & $0.0143(8)$ & $0.0264(10)$ & $0.0211(8)$ & $0.0080(7)$ & $-0.0022(7)$ & $-0.0023(7)$ \\
O3 & $0.0251(10)$ & $0.0369(12)$ & $0.0259(9)$ & $0.0147(9)$ & $0.0003(8)$ & $-0.0039(8)$ \\
N1 & $0.0280(12)$ & $0.0273(12)$ & $0.0217(11)$ & $0.0089(10)$ & $-0.0037(9)$ & $0.0022(9)$ \\
C1 & $0.0155(11)$ & $0.0237(13)$ & $0.0266(13)$ & $0.0072(10)$ & $-0.0008(10)$ & $0.0029(10)$ \\
C2 & $0.0184(12)$ & $0.0216(13)$ & $0.0306(13)$ & $0.0041(10)$ & $-0.0037(10)$ & $-0.0023(10)$ \\
C3 & $0.0172(12)$ & $0.0250(14)$ & $0.0341(14)$ & $0.0070(10)$ & $0.0015(11)$ & $0.0026(11)$ \\
C4 & $0.0247(13)$ & $0.0263(14)$ & $0.0302(14)$ & $0.0082(11)$ & $-0.0018(11)$ & $0.0069(11)$ \\
C5 & $0.0238(13)$ & $0.0256(14)$ & $0.0246(12)$ & $0.0077(10)$ & $-0.0021(10)$ & $0.0015(10)$ \\
C6 & $0.0176(11)$ & $0.0195(13)$ & $0.0243(12)$ & $0.0066(9)$ & $0.0003(10)$ & $0.0017(9)$ \\
C7 & $0.0155(11)$ & $0.0222(13)$ & $0.0220(12)$ & $0.0072(9)$ & $-0.0003(9)$ & $0.0004(9)$ \\
C8 & $0.0201(12)$ & $0.0231(13)$ & $0.0197(12)$ & $0.0094(10)$ & $-0.0015(9)$ & $0.0002(9)$ \\
C9 & $0.0269(13)$ & $0.0200(13)$ & $0.0251(13)$ & $0.0062(10)$ & $-0.0049(10)$ & $-0.0012(10)$ \\
C10 & $0.0275(13)$ & $0.0217(14)$ & $0.0272(13)$ & $0.0066(11)$ & $-0.0049(11)$ & $0.0013(10)$ \\
C11 & $0.0198(12)$ & $0.0262(14)$ & $0.0225(12)$ & $0.0106(10)$ & $-0.0022(10)$ & $0.0019(10)$ \\
C12 & $0.0196(12)$ & $0.0249(14)$ & $0.0213(12)$ & $0.0094(10)$ & $-0.0029(10)$ & $-0.0018(10)$ \\
C13 & $0.0151(11)$ & $0.0199(13)$ & $0.0248(12)$ & $0.0069(9)$ & $-0.0019(9)$ & $-0.0002(9)$ \\
C14 & $0.0206(12)$ & $0.0318(15)$ & $0.0236(13)$ & $0.0072(11)$ & $-0.0063(10)$ & $0.0032(11)$ \\
C15 & $0.0327(15)$ & $0.0360(17)$ & $0.0280(14)$ & $0.0104(13)$ & $-0.0036(12)$ & $-0.0021(11)$ \\
C16 & $0.0211(12)$ & $0.0316(15)$ & $0.0258(13)$ & $0.0050(11)$ & $-0.0012(10)$ & $0.0078(10)$ \\
C17 & $0.0331(16)$ & $0.0339(17)$ & $0.0481(18)$ & $0.0124(13)$ & $-0.0026(13)$ & $0.0138(13)$ \\
C18 & $0.0162(11)$ & $0.0200(12)$ & $0.0239(12)$ & $0.0050(9)$ & $-0.0041(9)$ & $0.0044(9)$ \\
C19 & $0.0203(12)$ & $0.0267(14)$ & $0.0337(14)$ & $0.0096(11)$ & $0.0009(11)$ & $0.0064(11)$
\end{tabular}




\begin{tabular}{lllllll}
\hline & & & & & \\
$\mathrm{C} 20$ & $0.0170(12)$ & $0.0315(16)$ & $0.0430(16)$ & $0.0067(11)$ & $-0.0027(11)$ & $0.0115(12)$ \\
$\mathrm{C} 21$ & $0.0198(13)$ & $0.0340(16)$ & $0.0325(14)$ & $-0.0010(11)$ & $-0.0100(11)$ & $0.0075(11)$ \\
$\mathrm{C} 22$ & $0.0263(14)$ & $0.0268(14)$ & $0.0208(12)$ & $0.0027(11)$ & $-0.0058(10)$ & $0.0019(10)$ \\
$\mathrm{C} 23$ & $0.0186(12)$ & $0.0223(13)$ & $0.0198(11)$ & $0.0065(10)$ & $-0.0019(9)$ & $0.0029(9)$ \\
$\mathrm{C} 24$ & $0.0197(12)$ & $0.0256(14)$ & $0.0186(12)$ & $0.0082(10)$ & $-0.0023(10)$ & $0.0007(9)$ \\
\hline
\end{tabular}

Geometric parameters $\left(\hat{A},{ }^{\circ}\right)$

\begin{tabular}{|c|c|c|c|}
\hline $\mathrm{Br} 1-\mathrm{C} 3$ & $1.898(3)$ & $\mathrm{C} 11-\mathrm{C} 12$ & $1.403(4)$ \\
\hline $\mathrm{O} 1-\mathrm{C} 1$ & $1.369(3)$ & $\mathrm{C} 12-\mathrm{C} 13$ & $1.383(4)$ \\
\hline $\mathrm{O} 1-\mathrm{C} 13$ & $1.381(3)$ & $\mathrm{C} 12-\mathrm{H} 12$ & 0.9500 \\
\hline $\mathrm{O} 2-\mathrm{C} 24$ & $1.362(3)$ & $\mathrm{C} 14-\mathrm{C} 15$ & $1.520(4)$ \\
\hline $\mathrm{O} 2-\mathrm{C} 7$ & $1.510(3)$ & $\mathrm{C} 14-\mathrm{H} 14 \mathrm{~A}$ & 0.9900 \\
\hline $\mathrm{O} 3-\mathrm{C} 24$ & $1.203(3)$ & C14-H14B & 0.9900 \\
\hline $\mathrm{N} 1-\mathrm{C} 11$ & $1.366(3)$ & C15-H15A & 0.9800 \\
\hline $\mathrm{N} 1-\mathrm{C} 16$ & $1.455(4)$ & C15-H15B & 0.9800 \\
\hline $\mathrm{N} 1-\mathrm{C} 14$ & $1.458(3)$ & $\mathrm{C} 15-\mathrm{H} 15 \mathrm{C}$ & 0.9800 \\
\hline $\mathrm{C} 1-\mathrm{C} 2$ & $1.384(4)$ & $\mathrm{C} 16-\mathrm{C} 17$ & $1.512(4)$ \\
\hline $\mathrm{C} 1-\mathrm{C} 6$ & $1.397(3)$ & C16-H16A & 0.9900 \\
\hline $\mathrm{C} 2-\mathrm{C} 3$ & $1.383(4)$ & C16-H16B & 0.9900 \\
\hline $\mathrm{C} 2-\mathrm{H} 2$ & 0.9500 & C17-H17A & 0.9800 \\
\hline $\mathrm{C} 3-\mathrm{C} 4$ & $1.393(4)$ & C17-H17B & 0.9800 \\
\hline $\mathrm{C} 4-\mathrm{C} 5$ & $1.384(4)$ & $\mathrm{C} 17-\mathrm{H} 17 \mathrm{C}$ & 0.9800 \\
\hline $\mathrm{C} 4-\mathrm{H} 4$ & 0.9500 & $\mathrm{C} 18-\mathrm{C} 23$ & $1.380(4)$ \\
\hline $\mathrm{C} 5-\mathrm{C} 6$ & $1.398(4)$ & $\mathrm{C} 18-\mathrm{C} 19$ & $1.386(4)$ \\
\hline $\mathrm{C} 5-\mathrm{H} 5$ & 0.9500 & $\mathrm{C} 19-\mathrm{C} 20$ & $1.398(4)$ \\
\hline $\mathrm{C} 6-\mathrm{C} 7$ & $1.502(4)$ & C19-H19 & 0.9500 \\
\hline $\mathrm{C} 7-\mathrm{C} 8$ & $1.496(4)$ & $\mathrm{C} 20-\mathrm{C} 21$ & $1.390(5)$ \\
\hline $\mathrm{C} 7-\mathrm{C} 18$ & $1.514(3)$ & $\mathrm{C} 20-\mathrm{H} 20$ & 0.9500 \\
\hline $\mathrm{C} 8-\mathrm{C} 13$ & $1.391(3)$ & $\mathrm{C} 21-\mathrm{C} 22$ & $1.384(4)$ \\
\hline $\mathrm{C} 8-\mathrm{C} 9$ & $1.402(4)$ & $\mathrm{C} 21-\mathrm{H} 21$ & 0.9500 \\
\hline $\mathrm{C} 9-\mathrm{C} 10$ & $1.373(4)$ & $\mathrm{C} 22-\mathrm{C} 23$ & $1.395(3)$ \\
\hline C9- $\mathrm{H} 9$ & 0.9500 & $\mathrm{C} 22-\mathrm{H} 22$ & 0.9500 \\
\hline $\mathrm{C} 10-\mathrm{C} 11$ & $1.420(4)$ & $\mathrm{C} 23-\mathrm{C} 24$ & $1.475(3)$ \\
\hline $\mathrm{C} 10-\mathrm{H} 10$ & 0.9500 & & \\
\hline $\mathrm{C} 1-\mathrm{O} 1-\mathrm{C} 13$ & $118.1(2)$ & $\mathrm{C} 12-\mathrm{C} 13-\mathrm{C} 8$ & $123.2(2)$ \\
\hline $\mathrm{C} 24-\mathrm{O} 2-\mathrm{C} 7$ & $111.26(18)$ & $\mathrm{N} 1-\mathrm{C} 14-\mathrm{C} 15$ & $112.8(2)$ \\
\hline $\mathrm{C} 11-\mathrm{N} 1-\mathrm{C} 16$ & $122.3(2)$ & $\mathrm{N} 1-\mathrm{C} 14-\mathrm{H} 14 \mathrm{~A}$ & 109.0 \\
\hline $\mathrm{C} 11-\mathrm{N} 1-\mathrm{C} 14$ & $121.8(2)$ & $\mathrm{C} 15-\mathrm{C} 14-\mathrm{H} 14 \mathrm{~A}$ & 109.0 \\
\hline $\mathrm{C} 16-\mathrm{N} 1-\mathrm{C} 14$ & $115.9(2)$ & $\mathrm{N} 1-\mathrm{C} 14-\mathrm{H} 14 \mathrm{~B}$ & 109.0 \\
\hline $\mathrm{O} 1-\mathrm{C} 1-\mathrm{C} 2$ & $115.3(2)$ & $\mathrm{C} 15-\mathrm{C} 14-\mathrm{H} 14 \mathrm{~B}$ & 109.0 \\
\hline $\mathrm{O} 1-\mathrm{C} 1-\mathrm{C} 6$ & $123.1(2)$ & $\mathrm{H} 14 \mathrm{~A}-\mathrm{C} 14-\mathrm{H} 14 \mathrm{~B}$ & 107.8 \\
\hline $\mathrm{C} 2-\mathrm{C} 1-\mathrm{C} 6$ & $121.6(2)$ & $\mathrm{C} 14-\mathrm{C} 15-\mathrm{H} 15 \mathrm{~A}$ & 109.5 \\
\hline $\mathrm{C} 3-\mathrm{C} 2-\mathrm{C} 1$ & $118.8(2)$ & $\mathrm{C} 14-\mathrm{C} 15-\mathrm{H} 15 \mathrm{~B}$ & 109.5 \\
\hline $\mathrm{C} 3-\mathrm{C} 2-\mathrm{H} 2$ & 120.6 & $\mathrm{H} 15 \mathrm{~A}-\mathrm{C} 15-\mathrm{H} 15 \mathrm{~B}$ & 109.5 \\
\hline $\mathrm{C} 1-\mathrm{C} 2-\mathrm{H} 2$ & 120.6 & $\mathrm{C} 14-\mathrm{C} 15-\mathrm{H} 15 \mathrm{C}$ & 109.5 \\
\hline $\mathrm{C} 2-\mathrm{C} 3-\mathrm{C} 4$ & $121.9(3)$ & $\mathrm{H} 15 \mathrm{~A}-\mathrm{C} 15-\mathrm{H} 15 \mathrm{C}$ & 109.5 \\
\hline
\end{tabular}




\begin{tabular}{|c|c|c|c|}
\hline $\mathrm{C} 2-\mathrm{C} 3-\mathrm{Br} 1$ & $119.1(2)$ & $\mathrm{H} 15 \mathrm{~B}-\mathrm{C} 15-\mathrm{H} 15 \mathrm{C}$ & 109.5 \\
\hline $\mathrm{C} 4-\mathrm{C} 3-\mathrm{Br} 1$ & $119.0(2)$ & $\mathrm{N} 1-\mathrm{C} 16-\mathrm{C} 17$ & $112.6(2)$ \\
\hline $\mathrm{C} 5-\mathrm{C} 4-\mathrm{C} 3$ & $117.9(3)$ & $\mathrm{N} 1-\mathrm{C} 16-\mathrm{H} 16 \mathrm{~A}$ & 109.1 \\
\hline $\mathrm{C} 5-\mathrm{C} 4-\mathrm{H} 4$ & 121.0 & $\mathrm{C} 17-\mathrm{C} 16-\mathrm{H} 16 \mathrm{~A}$ & 109.1 \\
\hline $\mathrm{C} 3-\mathrm{C} 4-\mathrm{H} 4$ & 121.0 & $\mathrm{~N} 1-\mathrm{C} 16-\mathrm{H} 16 \mathrm{~B}$ & 109.1 \\
\hline $\mathrm{C} 4-\mathrm{C} 5-\mathrm{C} 6$ & $122.2(3)$ & $\mathrm{C} 17-\mathrm{C} 16-\mathrm{H} 16 \mathrm{~B}$ & 109.1 \\
\hline $\mathrm{C} 4-\mathrm{C} 5-\mathrm{H} 5$ & 118.9 & $\mathrm{H} 16 \mathrm{~A}-\mathrm{C} 16-\mathrm{H} 16 \mathrm{~B}$ & 107.8 \\
\hline $\mathrm{C} 6-\mathrm{C} 5-\mathrm{H} 5$ & 118.9 & $\mathrm{C} 16-\mathrm{C} 17-\mathrm{H} 17 \mathrm{~A}$ & 109.5 \\
\hline $\mathrm{C} 1-\mathrm{C} 6-\mathrm{C} 5$ & $117.6(2)$ & $\mathrm{C} 16-\mathrm{C} 17-\mathrm{H} 17 \mathrm{~B}$ & 109.5 \\
\hline $\mathrm{C} 1-\mathrm{C} 6-\mathrm{C} 7$ & $120.7(2)$ & $\mathrm{H} 17 \mathrm{~A}-\mathrm{C} 17-\mathrm{H} 17 \mathrm{~B}$ & 109.5 \\
\hline $\mathrm{C} 5-\mathrm{C} 6-\mathrm{C} 7$ & $121.7(2)$ & $\mathrm{C} 16-\mathrm{C} 17-\mathrm{H} 17 \mathrm{C}$ & 109.5 \\
\hline $\mathrm{C} 8-\mathrm{C} 7-\mathrm{C} 6$ & $110.8(2)$ & $\mathrm{H} 17 \mathrm{~A}-\mathrm{C} 17-\mathrm{H} 17 \mathrm{C}$ & 109.5 \\
\hline $\mathrm{C} 8-\mathrm{C} 7-\mathrm{O} 2$ & $107.84(19)$ & $\mathrm{H} 17 \mathrm{~B}-\mathrm{C} 17-\mathrm{H} 17 \mathrm{C}$ & 109.5 \\
\hline $\mathrm{C} 6-\mathrm{C} 7-\mathrm{O} 2$ & $107.9(2)$ & $\mathrm{C} 23-\mathrm{C} 18-\mathrm{C} 19$ & $120.9(2)$ \\
\hline $\mathrm{C} 8-\mathrm{C} 7-\mathrm{C} 18$ & $114.7(2)$ & $\mathrm{C} 23-\mathrm{C} 18-\mathrm{C} 7$ & $110.1(2)$ \\
\hline $\mathrm{C} 6-\mathrm{C} 7-\mathrm{C} 18$ & $113.2(2)$ & $\mathrm{C} 19-\mathrm{C} 18-\mathrm{C} 7$ & $129.0(2)$ \\
\hline $\mathrm{O} 2-\mathrm{C} 7-\mathrm{C} 18$ & $101.64(19)$ & $\mathrm{C} 18-\mathrm{C} 19-\mathrm{C} 20$ & $117.7(3)$ \\
\hline $\mathrm{C} 13-\mathrm{C} 8-\mathrm{C} 9$ & $115.9(2)$ & $\mathrm{C} 18-\mathrm{C} 19-\mathrm{H} 19$ & 121.2 \\
\hline $\mathrm{C} 13-\mathrm{C} 8-\mathrm{C} 7$ & $121.6(2)$ & $\mathrm{C} 20-\mathrm{C} 19-\mathrm{H} 19$ & 121.2 \\
\hline $\mathrm{C} 9-\mathrm{C} 8-\mathrm{C} 7$ & $122.3(2)$ & $\mathrm{C} 21-\mathrm{C} 20-\mathrm{C} 19$ & $121.1(3)$ \\
\hline $\mathrm{C} 10-\mathrm{C} 9-\mathrm{C} 8$ & $122.5(2)$ & $\mathrm{C} 21-\mathrm{C} 20-\mathrm{H} 20$ & 119.4 \\
\hline $\mathrm{C} 10-\mathrm{C} 9-\mathrm{H} 9$ & 118.8 & $\mathrm{C} 19-\mathrm{C} 20-\mathrm{H} 20$ & 119.4 \\
\hline $\mathrm{C} 8-\mathrm{C} 9-\mathrm{H} 9$ & 118.8 & $\mathrm{C} 22-\mathrm{C} 21-\mathrm{C} 20$ & $121.0(2)$ \\
\hline $\mathrm{C} 9-\mathrm{C} 10-\mathrm{C} 11$ & $121.0(3)$ & $\mathrm{C} 22-\mathrm{C} 21-\mathrm{H} 21$ & 119.5 \\
\hline $\mathrm{C} 9-\mathrm{C} 10-\mathrm{H} 10$ & 119.5 & $\mathrm{C} 20-\mathrm{C} 21-\mathrm{H} 21$ & 119.5 \\
\hline $\mathrm{C} 11-\mathrm{C} 10-\mathrm{H} 10$ & 119.5 & $\mathrm{C} 21-\mathrm{C} 22-\mathrm{C} 23$ & $117.5(3)$ \\
\hline $\mathrm{N} 1-\mathrm{C} 11-\mathrm{C} 12$ & $122.0(2)$ & $\mathrm{C} 21-\mathrm{C} 22-\mathrm{H} 22$ & 121.3 \\
\hline $\mathrm{N} 1-\mathrm{C} 11-\mathrm{C} 10$ & $121.1(2)$ & $\mathrm{C} 23-\mathrm{C} 22-\mathrm{H} 22$ & 121.3 \\
\hline $\mathrm{C} 12-\mathrm{C} 11-\mathrm{C} 10$ & $116.9(2)$ & $\mathrm{C} 18-\mathrm{C} 23-\mathrm{C} 22$ & $121.8(2)$ \\
\hline $\mathrm{C} 13-\mathrm{C} 12-\mathrm{C} 11$ & $120.5(2)$ & $\mathrm{C} 18-\mathrm{C} 23-\mathrm{C} 24$ & $108.6(2)$ \\
\hline $\mathrm{C} 13-\mathrm{C} 12-\mathrm{H} 12$ & 119.7 & $\mathrm{C} 22-\mathrm{C} 23-\mathrm{C} 24$ & $129.6(2)$ \\
\hline $\mathrm{C} 11-\mathrm{C} 12-\mathrm{H} 12$ & 119.7 & $\mathrm{O} 3-\mathrm{C} 24-\mathrm{O} 2$ & $122.2(2)$ \\
\hline $\mathrm{O} 1-\mathrm{C} 13-\mathrm{C} 12$ & $114.7(2)$ & $\mathrm{O} 3-\mathrm{C} 24-\mathrm{C} 23$ & $129.5(2)$ \\
\hline $\mathrm{O} 1-\mathrm{C} 13-\mathrm{C} 8$ & $122.1(2)$ & $\mathrm{O} 2-\mathrm{C} 24-\mathrm{C} 23$ & $108.3(2)$ \\
\hline $\mathrm{C} 13-\mathrm{O} 1-\mathrm{C} 1-\mathrm{C} 2$ & $-165.8(2)$ & $\mathrm{C} 9-\mathrm{C} 10-\mathrm{C} 11-\mathrm{C} 12$ & $0.0(4)$ \\
\hline $\mathrm{C} 13-\mathrm{O} 1-\mathrm{C} 1-\mathrm{C} 6$ & $13.1(4)$ & $\mathrm{N} 1-\mathrm{C} 11-\mathrm{C} 12-\mathrm{C} 13$ & $179.1(2)$ \\
\hline $\mathrm{O} 1-\mathrm{C} 1-\mathrm{C} 2-\mathrm{C} 3$ & $179.4(2)$ & $\mathrm{C} 10-\mathrm{C} 11-\mathrm{C} 12-\mathrm{C} 13$ & $-1.0(4)$ \\
\hline $\mathrm{C} 6-\mathrm{C} 1-\mathrm{C} 2-\mathrm{C} 3$ & $0.5(4)$ & $\mathrm{C} 1-\mathrm{O} 1-\mathrm{C} 13-\mathrm{C} 12$ & $169.3(2)$ \\
\hline $\mathrm{C} 1-\mathrm{C} 2-\mathrm{C} 3-\mathrm{C} 4$ & $0.8(4)$ & $\mathrm{C} 1-\mathrm{O} 1-\mathrm{C} 13-\mathrm{C} 8$ & $-9.3(3)$ \\
\hline $\mathrm{C} 1-\mathrm{C} 2-\mathrm{C} 3-\mathrm{Br} 1$ & $-177.47(19)$ & $\mathrm{C} 11-\mathrm{C} 12-\mathrm{C} 13-\mathrm{O} 1$ & $-176.9(2)$ \\
\hline $\mathrm{C} 2-\mathrm{C} 3-\mathrm{C} 4-\mathrm{C} 5$ & $-1.3(4)$ & $\mathrm{C} 11-\mathrm{C} 12-\mathrm{C} 13-\mathrm{C} 8$ & $1.7(4)$ \\
\hline $\mathrm{Br} 1-\mathrm{C} 3-\mathrm{C} 4-\mathrm{C} 5$ & $176.9(2)$ & $\mathrm{C} 9-\mathrm{C} 8-\mathrm{C} 13-\mathrm{O} 1$ & $177.3(2)$ \\
\hline $\mathrm{C} 3-\mathrm{C} 4-\mathrm{C} 5-\mathrm{C} 6$ & $0.6(4)$ & $\mathrm{C} 7-\mathrm{C} 8-\mathrm{C} 13-\mathrm{O} 1$ & $-7.6(4)$ \\
\hline $\mathrm{O} 1-\mathrm{C} 1-\mathrm{C} 6-\mathrm{C} 5$ & $-180.0(2)$ & $\mathrm{C} 9-\mathrm{C} 8-\mathrm{C} 13-\mathrm{C} 12$ & $-1.2(4)$ \\
\hline $\mathrm{C} 2-\mathrm{C} 1-\mathrm{C} 6-\mathrm{C} 5$ & $-1.2(4)$ & $\mathrm{C} 7-\mathrm{C} 8-\mathrm{C} 13-\mathrm{C} 12$ & $173.9(2)$ \\
\hline $\mathrm{O} 1-\mathrm{C} 1-\mathrm{C} 6-\mathrm{C} 7$ & $0.1(4)$ & $\mathrm{C} 11-\mathrm{N} 1-\mathrm{C} 14-\mathrm{C} 15$ & $-87.3(3)$ \\
\hline $\mathrm{C} 2-\mathrm{C} 1-\mathrm{C} 6-\mathrm{C} 7$ & $178.9(2)$ & $\mathrm{C} 16-\mathrm{N} 1-\mathrm{C} 14-\mathrm{C} 15$ & $91.6(3)$ \\
\hline
\end{tabular}




$\begin{array}{llll}\mathrm{C} 4-\mathrm{C} 5-\mathrm{C} 6-\mathrm{C} 1 & 0.6(4) & \mathrm{C} 11-\mathrm{N} 1-\mathrm{C} 16-\mathrm{C} 17 & -87.7(3) \\ \mathrm{C} 4-\mathrm{C} 5-\mathrm{C} 6-\mathrm{C} 7 & -179.4(2) & \mathrm{C} 14-\mathrm{N} 1-\mathrm{C} 16-\mathrm{C} 17 & 93.4(3) \\ \mathrm{C} 1-\mathrm{C} 6-\mathrm{C} 7-\mathrm{C} 8 & -15.1(3) & \mathrm{C} 8-\mathrm{C} 7-\mathrm{C} 18-\mathrm{C} 23 & 115.2(2) \\ \mathrm{C} 5-\mathrm{C} 6-\mathrm{C} 7-\mathrm{C} 8 & 164.9(2) & \mathrm{C} 6-\mathrm{C} 7-\mathrm{C} 18-\mathrm{C} 23 & -116.3(2) \\ \mathrm{C} 1-\mathrm{C} 6-\mathrm{C} 7-\mathrm{O} 2 & 102.7(3) & \mathrm{O} 2-\mathrm{C} 7-\mathrm{C} 18-\mathrm{C} 23 & -0.9(3) \\ \mathrm{C} 5-\mathrm{C} 6-\mathrm{C} 7-\mathrm{O} 2 & -77.3(3) & \mathrm{C} 8-\mathrm{C} 7-\mathrm{C} 18-\mathrm{C} 19 & -66.8(4) \\ \mathrm{C} 1-\mathrm{C} 6-\mathrm{C} 7-\mathrm{C} 18 & -145.7(2) & \mathrm{C} 6-\mathrm{C} 7-\mathrm{C} 18-\mathrm{C} 19 & 61.7(4) \\ \mathrm{C} 5-\mathrm{C} 6-\mathrm{C} 7-\mathrm{C} 18 & 34.4(3) & \mathrm{O} 2-\mathrm{C} 7-\mathrm{C} 18-\mathrm{C} 19 & 177.1(3) \\ \mathrm{C} 24-\mathrm{O} 2-\mathrm{C} 7-\mathrm{C} 8 & -118.3(2) & \mathrm{C} 23-\mathrm{C} 18-\mathrm{C} 19-\mathrm{C} 20 & 1.8(4) \\ \mathrm{C} 24-\mathrm{O} 2-\mathrm{C} 7-\mathrm{C} 6 & 122.0(2) & \mathrm{C} 7-\mathrm{C} 18-\mathrm{C} 19-\mathrm{C} 20 & -176.0(3) \\ \mathrm{C} 24-\mathrm{O} 2-\mathrm{C} 7-\mathrm{C} 18 & 2.7(3) & \mathrm{C} 18-\mathrm{C} 19-\mathrm{C} 20-\mathrm{C} 21 & -1.4(4) \\ \mathrm{C} 6-\mathrm{C} 7-\mathrm{C} 8-\mathrm{C} 13 & 18.8(3) & \mathrm{C} 19-\mathrm{C} 20-\mathrm{C} 21-\mathrm{C} 22 & -0.5(5) \\ \mathrm{O} 2-\mathrm{C} 7-\mathrm{C} 8-\mathrm{C} 13 & -99.0(3) & \mathrm{C} 20-\mathrm{C} 21-\mathrm{C} 22-\mathrm{C} 23 & 1.9(4) \\ \mathrm{C} 18-\mathrm{C} 7-\mathrm{C} 8-\mathrm{C} 13 & 148.5(2) & \mathrm{C} 19-\mathrm{C} 18-\mathrm{C} 23-\mathrm{C} 22 & -0.4(4) \\ \mathrm{C} 6-\mathrm{C} 7-\mathrm{C} 8-\mathrm{C} 9 & -166.4(2) & \mathrm{C} 7-\mathrm{C} 18-\mathrm{C} 23-\mathrm{C} 22 & 177.8(2) \\ \mathrm{O} 2-\mathrm{C} 7-\mathrm{C} 8-\mathrm{C} 9 & 75.8(3) & \mathrm{C} 19-\mathrm{C} 18-\mathrm{C} 23-\mathrm{C} 24 & -179.2(2) \\ \mathrm{C} 18-\mathrm{C} 7-\mathrm{C} 8-\mathrm{C} 9 & -36.7(3) & \mathrm{C} 7-\mathrm{C} 18-\mathrm{C} 23-\mathrm{C} 24 & -1.0(3) \\ \mathrm{C} 13-\mathrm{C} 8-\mathrm{C} 9-\mathrm{C} 10 & 0.1(4) & \mathrm{C} 21-\mathrm{C} 22-\mathrm{C} 23-\mathrm{C} 18 & -1.5(4) \\ \mathrm{C} 7-\mathrm{C} 8-\mathrm{C} 9-\mathrm{C} 10 & -174.9(3) & \mathrm{C} 21-\mathrm{C} 22-\mathrm{C} 23-\mathrm{C} 24 & 177.1(3) \\ \mathrm{C} 8-\mathrm{C} 9-\mathrm{C} 10-\mathrm{C} 11 & 0.5(4) & \mathrm{C} 7-\mathrm{O} 2-\mathrm{C} 24-\mathrm{O} 3 & 177.5(2) \\ \mathrm{C} 16-\mathrm{N} 1-\mathrm{C} 11-\mathrm{C} 12 & -174.2(2) & \mathrm{C} 7-\mathrm{O} 2-\mathrm{C} 24-\mathrm{C} 23 & -3.4(3) \\ \mathrm{C} 14-\mathrm{N} 1-\mathrm{C} 11-\mathrm{C} 12 & 4.7(4) & \mathrm{C} 18-\mathrm{C} 23-\mathrm{C} 24-\mathrm{O} 3 & -178.2(3) \\ \mathrm{C} 16-\mathrm{N} 1-\mathrm{C} 11-\mathrm{C} 10 & 5.9(4) & \mathrm{C} 22-\mathrm{C} 23-\mathrm{C} 24-\mathrm{O} 3 & 3.1(5) \\ \mathrm{C} 14-\mathrm{N} 1-\mathrm{C} 11-\mathrm{C} 10 & -175.2(2) & \mathrm{C} 18-\mathrm{C} 23-\mathrm{C} 24-\mathrm{O} 2 & 2.7(3) \\ \mathrm{C} 9-\mathrm{C} 10-\mathrm{C} 11-\mathrm{N} 1 & 179.9(3) & \mathrm{C} 22-\mathrm{C} 23-\mathrm{C} 24-\mathrm{O} 2 & -175.9(3) \\ & & & \end{array}$

Hydrogen-bond geometry $\left(\AA,{ }^{\circ}\right)$

$\mathrm{Cg}$ is the centroid of the $\mathrm{C} 8-\mathrm{C} 13$ ring.

\begin{tabular}{lllll}
\hline$D-\mathrm{H} \cdots A$ & $D-\mathrm{H}$ & $\mathrm{H} \cdots A$ & $D \cdots A$ & $D-\mathrm{H} \cdots A$ \\
\hline $\mathrm{C} 14-\mathrm{H} 14 B \cdots \mathrm{O} 2^{\mathrm{i}}$ & 0.99 & 2.68 & $3.649(4)$ & 165 \\
$\mathrm{C} 16-\mathrm{H} 16 A \cdots 3^{\mathrm{ii}}$ & 0.99 & 2.64 & $3.522(3)$ & 148 \\
$\mathrm{C} 16-\mathrm{H} 16 B \cdots B^{\mathrm{ii}} 1^{\mathrm{iii}}$ & 0.99 & 2.99 & $3.939(3)$ & 162 \\
$\mathrm{C} 17-\mathrm{H} 17 A \cdots C g^{\mathrm{iv}}$ & 0.98 & 2.75 & $3.666(4)$ & 156 \\
$\mathrm{C} 19-\mathrm{H} 19 \cdots{ }^{\mathrm{iii}}$ & 0.95 & 2.57 & $3.485(4)$ & 161 \\
$\mathrm{C} 20-\mathrm{H} 20 \cdots \mathrm{O}^{\mathrm{ii}}$ & 0.95 & 2.58 & $3.421(3)$ & 148
\end{tabular}

Symmetry codes: (i) $-x,-y+1,-z+1$; (ii) $x, y, z-1$; (iii) $-x+1,-y+1,-z+1$; (iv) $-x+1,-y+2,-z+1$; (v) $x+1, y, z$.

(4) (1S)-6'-Bromo-3'-diethylamino-3H-spiro[2-benzofuran-1,9'-xanthen]-3-one

Crystal data

$\mathrm{C}_{24} \mathrm{H}_{20} \mathrm{BrNO}_{3}$

$M_{r}=450.32$

Orthorhombic, $P 2_{1} 2_{1} 2_{1}$

$a=11.0772(6) \AA$

$b=13.0582(8) \AA$

$c=13.8966(8) \AA$

$V=2010.1(2) \AA^{3}$
$Z=4$

$F(000)=920$

$D_{\mathrm{x}}=1.488 \mathrm{Mg} \mathrm{m}^{-3}$

Mo $K \alpha$ radiation, $\lambda=0.71073 \AA$

Cell parameters from 9874 reflections

$\theta=2.4-28.7^{\circ}$

$\mu=2.07 \mathrm{~mm}^{-1}$ 
$T=100 \mathrm{~K}$

Column, colourless

\section{Data collection}

\section{Bruker SMART APEX CCD diffractometer}

Radiation source: fine-focus sealed tube

Graphite monochromator

Detector resolution: 8.3333 pixels $\mathrm{mm}^{-1}$

$\varphi$ and $\omega$ scans

Absorption correction: multi-scan

(SADABS; Bruker, 2016)

$T_{\min }=0.69, T_{\max }=0.82$

Refinement

Refinement on $F^{2}$

Least-squares matrix: full

$R\left[F^{2}>2 \sigma\left(F^{2}\right)\right]=0.026$

$w R\left(F^{2}\right)=0.058$

$S=0.99$

5417 reflections

320 parameters

0 restraints

Primary atom site location: structure-invariant direct methods

Secondary atom site location: difference Fourier map
$0.31 \times 0.12 \times 0.10 \mathrm{~mm}$

39006 measured reflections

5417 independent reflections

4926 reflections with $I>2 \sigma(I)$

$R_{\text {int }}=0.043$

$\theta_{\max }=29.2^{\circ}, \theta_{\min }=2.1^{\circ}$

$h=-15 \rightarrow 15$

$k=-17 \rightarrow 17$

$l=-18 \rightarrow 18$

Hydrogen site location: mixed

$\mathrm{H}$ atoms treated by a mixture of independent and constrained refinement

$w=1 /\left[\sigma^{2}\left(F_{\mathrm{o}}^{2}\right)+(0.0299 P)^{2}\right]$

where $P=\left(F_{\mathrm{o}}{ }^{2}+2 F_{\mathrm{c}}{ }^{2}\right) / 3$

$(\Delta / \sigma)_{\max }=0.001$

$\Delta \rho_{\max }=0.62 \mathrm{e}^{-3}$

$\Delta \rho_{\min }=-0.25$ e $\AA^{-3}$

Absolute structure: Flack $x$ determined using 1981 quotients $\left[\left(I^{+}\right)-(I)\right] /\left[\left(I^{+}\right)+\left(I^{-}\right)\right.$(Parsons et al., 2013)

Absolute structure parameter: $-0.006(3)$

\section{Special details}

Experimental. The diffraction data were obtained from 3 sets of 400 frames, each of width $0.5^{\circ}$ in $\omega$, colllected at $\varphi=$ $0.00,90.00$ and $180.00^{\circ}$ and 2 sets of 800 frames, each of width $0.45^{\circ}$ in $\varphi$, collected at $\omega=-30.00$ and $210.00^{\circ}$. The scan time was $20 \mathrm{sec} /$ frame.

Geometry. All esds (except the esd in the dihedral angle between two 1.s. planes) are estimated using the full covariance matrix. The cell esds are taken into account individually in the estimation of esds in distances, angles and torsion angles; correlations between esds in cell parameters are only used when they are defined by crystal symmetry. An approximate (isotropic) treatment of cell esds is used for estimating esds involving l.s. planes.

Refinement. Refinement of $\mathrm{F}^{2}$ against ALL reflections. The weighted R-factor $\mathrm{wR}$ and goodness of fit $\mathrm{S}$ are based on $\mathrm{F}^{2}$, conventional R-factors $R$ are based on $F$, with $F$ set to zero for negative $F^{2}$. The threshold expression of $F^{2}>2 \operatorname{sigma}\left(\mathrm{F}^{2}\right)$ is used only for calculating R-factors(gt) etc. and is not relevant to the choice of reflections for refinement. R-factors based on $\mathrm{F}^{2}$ are statistically about twice as large as those based on $\mathrm{F}$, and $\mathrm{R}$ - factors based on ALL data will be even larger.

Fractional atomic coordinates and isotropic or equivalent isotropic displacement parameters $\left(\AA^{2}\right)$

\begin{tabular}{lllll}
\hline & $x$ & $y$ & $z$ & $U_{\text {iso }} * / U_{\text {eq }}$ \\
\hline Br1 & $0.23778(2)$ & $0.57329(2)$ & $0.58190(2)$ & $0.02156(7)$ \\
O1 & $0.62242(14)$ & $0.63049(13)$ & $0.37859(12)$ & $0.0174(4)$ \\
O2 & $0.83447(14)$ & $0.44945(12)$ & $0.52206(11)$ & $0.0149(3)$ \\
O3 & $0.96324(16)$ & $0.34761(13)$ & $0.60162(12)$ & $0.0232(4)$ \\
N1 & $0.93770(19)$ & $0.75727(16)$ & $0.17844(14)$ & $0.0191(4)$ \\
C1 & $0.5808(2)$ & $0.59393(17)$ & $0.46462(16)$ & $0.0132(5)$ \\
C2 & $0.4557(2)$ & $0.59644(18)$ & $0.47625(18)$ & $0.0166(5)$ \\
H2 & $0.412(3)$ & $0.617(2)$ & $0.427(2)$ & $0.023(7)^{*}$ \\
C3 & $0.40723(19)$ & $0.56383(17)$ & $0.56230(16)$ & $0.0154(5)$
\end{tabular}




\begin{tabular}{|c|c|c|c|c|}
\hline $\mathrm{C} 4$ & $0.4786(2)$ & $0.52661(19)$ & $0.63625(18)$ & $0.0178(5)$ \\
\hline $\mathrm{H} 4$ & $0.440(2)$ & $0.5053(19)$ & $0.693(2)$ & $0.017(7)^{*}$ \\
\hline $\mathrm{C} 5$ & $0.6020(2)$ & $0.52317(19)$ & $0.62259(18)$ & $0.0163(5)$ \\
\hline H5 & $0.651(3)$ & $0.495(2)$ & $0.6671(19)$ & $0.020(8)^{*}$ \\
\hline C6 & $0.6556(2)$ & $0.55733(18)$ & $0.53668(17)$ & $0.0138(5)$ \\
\hline $\mathrm{C} 7$ & 0.79072 (19) & $0.55778(16)$ & $0.52627(16)$ & $0.0126(4)$ \\
\hline $\mathrm{C} 8$ & $0.8285(2)$ & $0.61105(17)$ & $0.43527(16)$ & $0.0132(4)$ \\
\hline C9 & $0.9502(2)$ & $0.63061(18)$ & $0.41480(19)$ & $0.0170(5)$ \\
\hline H9 & $1.007(2)$ & 0.6057 (19) & $0.4633(18)$ & $0.012(6)^{*}$ \\
\hline $\mathrm{C} 10$ & $0.9872(2)$ & 0.67728 (19) & $0.33110(18)$ & $0.0178(5)$ \\
\hline $\mathrm{H} 10$ & $1.070(3)$ & $0.685(2)$ & $0.3211(19)$ & $0.020(7)^{*}$ \\
\hline $\mathrm{C} 11$ & $0.9017(2)$ & $0.70912(17)$ & $0.26139(16)$ & $0.0148(5)$ \\
\hline $\mathrm{C} 12$ & $0.7793(2)$ & $0.68911(17)$ & $0.28086(17)$ & $0.0152(5)$ \\
\hline $\mathrm{H} 12$ & $0.722(2)$ & $0.7083(19)$ & $0.2415(17)$ & $0.013(6)^{*}$ \\
\hline $\mathrm{C} 13$ & $0.7462(2)$ & $0.64179(15)$ & $0.36616(15)$ & $0.0129(4)$ \\
\hline $\mathrm{C} 14$ & $0.8504(2)$ & $0.7873(2)$ & $0.10481(18)$ & $0.0227(5)$ \\
\hline $\mathrm{H} 14 \mathrm{~A}$ & $0.787(3)$ & $0.733(2)$ & $0.098(2)$ & $0.031(8)^{*}$ \\
\hline H14B & $0.891(2)$ & $0.789(2)$ & $0.0417(18)$ & $0.014(6)^{*}$ \\
\hline $\mathrm{C} 15$ & $0.7924(2)$ & $0.8904(2)$ & $0.1260(2)$ & $0.0285(6)$ \\
\hline $\mathrm{H} 15 \mathrm{~A}$ & 0.7563 & 0.8890 & 0.1903 & $0.043^{*}$ \\
\hline H15B & 0.7296 & 0.9043 & 0.0781 & $0.043^{*}$ \\
\hline $\mathrm{H} 15 \mathrm{C}$ & 0.8539 & 0.9442 & 0.1231 & $0.043^{*}$ \\
\hline $\mathrm{C} 16$ & $1.0602(2)$ & $0.7975(2)$ & $0.16496(19)$ & $0.0198(5)$ \\
\hline H16A & $1.047(2)$ & $0.866(2)$ & $0.1302(18)$ & $0.017(7)^{*}$ \\
\hline H16B & $1.089(2)$ & $0.820(2)$ & $0.230(2)$ & $0.021(7)^{*}$ \\
\hline C17 & $1.1432(2)$ & $0.7252(2)$ & $0.1109(2)$ & $0.0253(6)$ \\
\hline H17A & 1.1564 & 0.6633 & 0.1493 & $0.038^{*}$ \\
\hline H17B & 1.2208 & 0.7591 & 0.0990 & $0.038^{*}$ \\
\hline $\mathrm{H} 17 \mathrm{C}$ & 1.1062 & 0.7066 & 0.0493 & $0.038^{*}$ \\
\hline C18 & $0.8524(2)$ & $0.59745(18)$ & $0.61583(17)$ & $0.0139(5)$ \\
\hline C19 & $0.8402(2)$ & $0.69192(19)$ & $0.65983(18)$ & $0.0179(5)$ \\
\hline H19 & $0.788(2)$ & $0.739(2)$ & $0.6336(19)$ & $0.019(7)^{*}$ \\
\hline $\mathrm{C} 20$ & $0.9055(2)$ & $0.7091(2)$ & $0.74369(19)$ & $0.0228(5)$ \\
\hline $\mathrm{H} 20$ & $0.909(3)$ & $0.770(3)$ & $0.779(2)$ & $0.039(9)^{*}$ \\
\hline $\mathrm{C} 21$ & $0.9793(2)$ & $0.6332(2)$ & $0.78279(19)$ & $0.0230(6)$ \\
\hline $\mathrm{H} 21$ & $1.024(2)$ & $0.649(2)$ & $0.8452(19)$ & $0.021(7)^{*}$ \\
\hline $\mathrm{C} 22$ & $0.9906(2)$ & $0.5390(2)$ & $0.73893(18)$ & $0.0202(5)$ \\
\hline $\mathrm{H} 22$ & $1.040(2)$ & $0.4897(19)$ & $0.7606(19)$ & $0.013(7)^{*}$ \\
\hline $\mathrm{C} 23$ & $0.9256(2)$ & $0.52284(19)$ & $0.65427(18)$ & $0.0158(5)$ \\
\hline $\mathrm{C} 24$ & $0.91472(18)$ & $0.42975(18)$ & $0.59411(15)$ & $0.0151(4)$ \\
\hline
\end{tabular}

Atomic displacement parameters $\left(\AA^{2}\right)$

\begin{tabular}{lllllll}
\hline & $U^{11}$ & $U^{22}$ & $U^{33}$ & $U^{12}$ & $U^{13}$ & $U^{23}$ \\
\hline Br1 & $0.01162(10)$ & $0.03082(12)$ & $0.02224(12)$ & $-0.00078(9)$ & $0.00204(9)$ & $-0.00572(10)$ \\
O1 & $0.0111(8)$ & $0.0258(9)$ & $0.0152(8)$ & $-0.0016(7)$ & $-0.0013(7)$ & $0.0059(7)$ \\
O2 & $0.0166(8)$ & $0.0134(8)$ & $0.0148(8)$ & $0.0025(6)$ & $-0.0012(6)$ & $0.0002(6)$ \\
O3 & $0.0250(9)$ & $0.0233(9)$ & $0.0215(10)$ & $0.0091(7)$ & $-0.0017(8)$ & $0.0044(7)$
\end{tabular}




\begin{tabular}{lllllll}
$\mathrm{N} 1$ & $0.0158(10)$ & $0.0272(11)$ & $0.0142(10)$ & $-0.0032(9)$ & $0.0012(8)$ & $0.0036(9)$ \\
$\mathrm{C} 1$ & $0.0139(11)$ & $0.0135(11)$ & $0.0123(11)$ & $-0.0014(8)$ & $0.0008(9)$ & $-0.0005(8)$ \\
$\mathrm{C} 2$ & $0.0135(11)$ & $0.0208(12)$ & $0.0155(12)$ & $0.0006(9)$ & $-0.0034(9)$ & $0.0008(9)$ \\
$\mathrm{C} 3$ & $0.0106(9)$ & $0.0156(10)$ & $0.0199(12)$ & $-0.0024(9)$ & $0.0005(8)$ & $-0.0047(10)$ \\
$\mathrm{C} 4$ & $0.0176(12)$ & $0.0199(12)$ & $0.0161(12)$ & $-0.0039(9)$ & $0.0022(10)$ & $0.0019(10)$ \\
$\mathrm{C} 5$ & $0.0159(12)$ & $0.0192(11)$ & $0.0138(12)$ & $-0.0002(9)$ & $-0.0023(9)$ & $0.0025(10)$ \\
C6 & $0.0121(10)$ & $0.0130(11)$ & $0.0162(11)$ & $0.0002(9)$ & $0.0005(9)$ & $0.0008(9)$ \\
C7 & $0.0127(10)$ & $0.0124(10)$ & $0.0127(10)$ & $0.0014(8)$ & $-0.0002(8)$ & $0.0001(8)$ \\
C8 & $0.0125(10)$ & $0.0159(10)$ & $0.0111(11)$ & $0.0020(8)$ & $0.0004(8)$ & $0.0009(9)$ \\
C9 & $0.0132(10)$ & $0.0221(11)$ & $0.0157(11)$ & $0.0019(9)$ & $-0.0022(11)$ & $0.0012(11)$ \\
C10 & $0.0119(11)$ & $0.0243(13)$ & $0.0173(12)$ & $-0.0011(10)$ & $0.0021(9)$ & $0.0009(10)$ \\
C11 & $0.0176(11)$ & $0.0146(10)$ & $0.0123(11)$ & $0.0002(9)$ & $0.0021(9)$ & $-0.0019(9)$ \\
C12 & $0.0148(11)$ & $0.0179(11)$ & $0.0130(11)$ & $0.0005(9)$ & $-0.0024(9)$ & $0.0006(8)$ \\
C13 & $0.0099(10)$ & $0.0143(9)$ & $0.0146(10)$ & $0.0000(9)$ & $-0.0001(9)$ & $-0.0019(8)$ \\
C14 & $0.0230(13)$ & $0.0319(14)$ & $0.0133(12)$ & $-0.0058(11)$ & $-0.0017(10)$ & $0.0062(10)$ \\
C15 & $0.0240(14)$ & $0.0340(14)$ & $0.0275(14)$ & $-0.0012(11)$ & $-0.0029(11)$ & $0.0130(12)$ \\
C16 & $0.0191(12)$ & $0.0241(13)$ & $0.0162(12)$ & $-0.0046(10)$ & $0.0026(10)$ & $0.0020(10)$ \\
C17 & $0.0207(12)$ & $0.0305(14)$ & $0.0248(14)$ & $-0.0004(11)$ & $0.0038(10)$ & $-0.0014(11)$ \\
C18 & $0.0092(10)$ & $0.0201(11)$ & $0.0124(10)$ & $-0.0034(8)$ & $0.0010(8)$ & $0.0010(9)$ \\
C19 & $0.0150(12)$ & $0.0175(12)$ & $0.0210(13)$ & $-0.0009(9)$ & $0.0006(10)$ & $-0.0012(10)$ \\
C20 & $0.0182(12)$ & $0.0271(14)$ & $0.0231(14)$ & $-0.0071(11)$ & $0.0028(10)$ & $-0.0078(11)$ \\
C21 & $0.0164(12)$ & $0.0373(15)$ & $0.0152(12)$ & $-0.0080(11)$ & $-0.0015(10)$ & $-0.0020(11)$ \\
C22 & $0.0128(11)$ & $0.0318(14)$ & $0.0159(12)$ & $-0.0015(10)$ & $-0.0009(10)$ & $0.0062(10)$ \\
C23 & $0.0116(11)$ & $0.0210(12)$ & $0.0149(12)$ & $-0.0004(9)$ & $0.0017(9)$ & $0.0033(10)$ \\
C24 & $0.0120(9)$ & $0.0199(10)$ & $0.0133(11)$ & $0.0011(9)$ & $0.0024(8)$ & $0.0033(10)$ \\
& & & & & & \\
\hline
\end{tabular}

Geometric parameters $\left(\AA,{ }^{\circ}\right)$

\begin{tabular}{llll}
\hline $\mathrm{Br} 1-\mathrm{C} 3$ & $1.901(2)$ & $\mathrm{C} 11-\mathrm{C} 12$ & $1.408(3)$ \\
$\mathrm{O} 1-\mathrm{C} 1$ & $1.367(3)$ & $\mathrm{C} 12-\mathrm{C} 13$ & $1.386(3)$ \\
$\mathrm{O} 1-\mathrm{C} 13$ & $1.389(3)$ & $\mathrm{C} 12-\mathrm{H} 12$ & $0.87(2)$ \\
$\mathrm{O} 2-\mathrm{C} 24$ & $1.363(3)$ & $\mathrm{C} 14-\mathrm{C} 15$ & $1.520(4)$ \\
$\mathrm{O} 2-\mathrm{C} 7$ & $1.496(3)$ & $\mathrm{C} 14-\mathrm{H} 14 \mathrm{~A}$ & $1.01(3)$ \\
$\mathrm{O} 3-\mathrm{C} 24$ & $1.204(3)$ & $\mathrm{C} 14-\mathrm{H} 14 \mathrm{~B}$ & $0.98(3)$ \\
$\mathrm{N} 1-\mathrm{C} 11$ & $1.372(3)$ & $\mathrm{C} 15-\mathrm{H} 15 \mathrm{~A}$ & 0.9800 \\
$\mathrm{~N} 1-\mathrm{C} 14$ & $1.461(3)$ & $\mathrm{C} 15-\mathrm{H} 15 \mathrm{~B}$ & 0.9800 \\
$\mathrm{~N} 1-\mathrm{C} 16$ & $1.467(3)$ & $\mathrm{C} 15-\mathrm{H} 15 \mathrm{C}$ & 0.9800 \\
$\mathrm{C} 1-\mathrm{C} 6$ & $1.385(3)$ & $\mathrm{C} 16-\mathrm{C} 17$ & $1.517(4)$ \\
$\mathrm{C} 1-\mathrm{C} 2$ & $1.395(3)$ & $\mathrm{C} 16-\mathrm{H} 16 \mathrm{~A}$ & $1.02(3)$ \\
$\mathrm{C} 2-\mathrm{C} 3$ & $1.378(3)$ & $\mathrm{C} 16-\mathrm{H} 16 \mathrm{~B}$ & $0.99(3)$ \\
$\mathrm{C} 2-\mathrm{H} 2$ & $0.88(3)$ & $\mathrm{C} 17-\mathrm{H} 17 \mathrm{~A}$ & 0.9800 \\
$\mathrm{C} 3-\mathrm{C} 4$ & $1.385(3)$ & $\mathrm{C} 17-\mathrm{H} 17 \mathrm{~B}$ & 0.9800 \\
$\mathrm{C} 4-\mathrm{C} 5$ & $1.381(4)$ & $\mathrm{C} 17-\mathrm{H} 17 \mathrm{C}$ & 0.9800 \\
$\mathrm{C} 4-\mathrm{H} 4$ & $0.93(3)$ & $\mathrm{C} 18-\mathrm{C} 23$ & $1.375(3)$ \\
$\mathrm{C} 5-\mathrm{C} 6$ & $1.406(3)$ & $\mathrm{C} 18-\mathrm{C} 19$ & $1.384(3)$ \\
$\mathrm{C} 5-\mathrm{H} 5$ & $0.90(3)$ & $\mathrm{C} 19-\mathrm{C} 20$ & $1.390(3)$ \\
$\mathrm{C} 6-\mathrm{C} 7$ & $1.504(3)$ & $\mathrm{C} 19-\mathrm{H} 19$ & $0.92(3)$ \\
$\mathrm{C} 7-\mathrm{C} 8$ & $1.503(3)$ & $\mathrm{C} 20-\mathrm{C} 21$ & $1.395(4)$
\end{tabular}




\begin{tabular}{|c|c|c|c|}
\hline $\mathrm{C} 7-\mathrm{C} 18$ & $1.512(3)$ & $\mathrm{C} 20-\mathrm{H} 20$ & $0.93(3)$ \\
\hline $\mathrm{C} 8-\mathrm{C} 13$ & $1.384(3)$ & $\mathrm{C} 21-\mathrm{C} 22$ & $1.378(4)$ \\
\hline $\mathrm{C} 8-\mathrm{C} 9$ & $1.401(3)$ & $\mathrm{C} 21-\mathrm{H} 21$ & $1.02(3)$ \\
\hline $\mathrm{C} 9-\mathrm{C} 10$ & $1.376(3)$ & $\mathrm{C} 22-\mathrm{C} 23$ & $1.396(3)$ \\
\hline $\mathrm{C} 9-\mathrm{H} 9$ & $0.98(3)$ & $\mathrm{C} 22-\mathrm{H} 22$ & $0.90(3)$ \\
\hline $\mathrm{C} 10-\mathrm{C} 11$ & $1.417(3)$ & $\mathrm{C} 23-\mathrm{C} 24$ & $1.480(3)$ \\
\hline $\mathrm{C} 10-\mathrm{H} 10$ & $0.93(3)$ & & \\
\hline $\mathrm{C} 1-\mathrm{O} 1-\mathrm{C} 13$ & $118.57(17)$ & $\mathrm{C} 12-\mathrm{C} 13-\mathrm{O} 1$ & $114.49(19)$ \\
\hline $\mathrm{C} 24-\mathrm{O} 2-\mathrm{C} 7$ & $111.15(17)$ & $\mathrm{N} 1-\mathrm{C} 14-\mathrm{C} 15$ & $112.5(2)$ \\
\hline $\mathrm{C} 11-\mathrm{N} 1-\mathrm{C} 14$ & $121.3(2)$ & $\mathrm{N} 1-\mathrm{C} 14-\mathrm{H} 14 \mathrm{~A}$ & $109.6(17)$ \\
\hline $\mathrm{C} 11-\mathrm{N} 1-\mathrm{C} 16$ & $122.7(2)$ & $\mathrm{C} 15-\mathrm{C} 14-\mathrm{H} 14 \mathrm{~A}$ & $110.3(16)$ \\
\hline $\mathrm{C} 14-\mathrm{N} 1-\mathrm{C} 16$ & $115.2(2)$ & $\mathrm{N} 1-\mathrm{C} 14-\mathrm{H} 14 \mathrm{~B}$ & $109.3(15)$ \\
\hline $\mathrm{O} 1-\mathrm{C} 1-\mathrm{C} 6$ & $123.4(2)$ & $\mathrm{C} 15-\mathrm{C} 14-\mathrm{H} 14 \mathrm{~B}$ & $110.3(15)$ \\
\hline $\mathrm{O} 1-\mathrm{C} 1-\mathrm{C} 2$ & $115.3(2)$ & $\mathrm{H} 14 \mathrm{~A}-\mathrm{C} 14-\mathrm{H} 14 \mathrm{~B}$ & $105(2)$ \\
\hline $\mathrm{C} 6-\mathrm{C} 1-\mathrm{C} 2$ & $121.2(2)$ & $\mathrm{C} 14-\mathrm{C} 15-\mathrm{H} 15 \mathrm{~A}$ & 109.5 \\
\hline $\mathrm{C} 3-\mathrm{C} 2-\mathrm{C} 1$ & $118.7(2)$ & $\mathrm{C} 14-\mathrm{C} 15-\mathrm{H} 15 \mathrm{~B}$ & 109.5 \\
\hline $\mathrm{C} 3-\mathrm{C} 2-\mathrm{H} 2$ & $123.4(18)$ & $\mathrm{H} 15 \mathrm{~A}-\mathrm{C} 15-\mathrm{H} 15 \mathrm{~B}$ & 109.5 \\
\hline $\mathrm{C} 1-\mathrm{C} 2-\mathrm{H} 2$ & $117.9(18)$ & $\mathrm{C} 14-\mathrm{C} 15-\mathrm{H} 15 \mathrm{C}$ & 109.5 \\
\hline $\mathrm{C} 2-\mathrm{C} 3-\mathrm{C} 4$ & $122.0(2)$ & $\mathrm{H} 15 \mathrm{~A}-\mathrm{C} 15-\mathrm{H} 15 \mathrm{C}$ & 109.5 \\
\hline $\mathrm{C} 2-\mathrm{C} 3-\mathrm{Br} 1$ & $119.29(17)$ & $\mathrm{H} 15 \mathrm{~B}-\mathrm{C} 15-\mathrm{H} 15 \mathrm{C}$ & 109.5 \\
\hline $\mathrm{C} 4-\mathrm{C} 3-\mathrm{Br} 1$ & $118.69(17)$ & $\mathrm{N} 1-\mathrm{C} 16-\mathrm{C} 17$ & $113.6(2)$ \\
\hline $\mathrm{C} 5-\mathrm{C} 4-\mathrm{C} 3$ & $118.3(2)$ & $\mathrm{N} 1-\mathrm{C} 16-\mathrm{H} 16 \mathrm{~A}$ & $104.1(15)$ \\
\hline $\mathrm{C} 5-\mathrm{C} 4-\mathrm{H} 4$ & $123.7(17)$ & $\mathrm{C} 17-\mathrm{C} 16-\mathrm{H} 16 \mathrm{~A}$ & $113.0(15)$ \\
\hline $\mathrm{C} 3-\mathrm{C} 4-\mathrm{H} 4$ & $118.0(17)$ & $\mathrm{N} 1-\mathrm{C} 16-\mathrm{H} 16 \mathrm{~B}$ & $106.5(16)$ \\
\hline $\mathrm{C} 4-\mathrm{C} 5-\mathrm{C} 6$ & $121.6(2)$ & $\mathrm{C} 17-\mathrm{C} 16-\mathrm{H} 16 \mathrm{~B}$ & $115.7(16)$ \\
\hline $\mathrm{C} 4-\mathrm{C} 5-\mathrm{H} 5$ & $120.9(19)$ & $\mathrm{H} 16 \mathrm{~A}-\mathrm{C} 16-\mathrm{H} 16 \mathrm{~B}$ & $103(2)$ \\
\hline $\mathrm{C} 6-\mathrm{C} 5-\mathrm{H} 5$ & $117.3(19)$ & $\mathrm{C} 16-\mathrm{C} 17-\mathrm{H} 17 \mathrm{~A}$ & 109.5 \\
\hline $\mathrm{C} 1-\mathrm{C} 6-\mathrm{C} 5$ & $118.1(2)$ & $\mathrm{C} 16-\mathrm{C} 17-\mathrm{H} 17 \mathrm{~B}$ & 109.5 \\
\hline $\mathrm{C} 1-\mathrm{C} 6-\mathrm{C} 7$ & $121.6(2)$ & $\mathrm{H} 17 \mathrm{~A}-\mathrm{C} 17-\mathrm{H} 17 \mathrm{~B}$ & 109.5 \\
\hline $\mathrm{C} 5-\mathrm{C} 6-\mathrm{C} 7$ & $120.2(2)$ & $\mathrm{C} 16-\mathrm{C} 17-\mathrm{H} 17 \mathrm{C}$ & 109.5 \\
\hline $\mathrm{O} 2-\mathrm{C} 7-\mathrm{C} 8$ & $108.32(17)$ & $\mathrm{H} 17 \mathrm{~A}-\mathrm{C} 17-\mathrm{H} 17 \mathrm{C}$ & 109.5 \\
\hline $\mathrm{O} 2-\mathrm{C} 7-\mathrm{C} 6$ & $108.81(18)$ & $\mathrm{H} 17 \mathrm{~B}-\mathrm{C} 17-\mathrm{H} 17 \mathrm{C}$ & 109.5 \\
\hline $\mathrm{C} 8-\mathrm{C} 7-\mathrm{C} 6$ & $111.12(19)$ & $\mathrm{C} 23-\mathrm{C} 18-\mathrm{C} 19$ & $121.2(2)$ \\
\hline $\mathrm{O} 2-\mathrm{C} 7-\mathrm{C} 18$ & $102.10(17)$ & $\mathrm{C} 23-\mathrm{C} 18-\mathrm{C} 7$ & $110.1(2)$ \\
\hline $\mathrm{C} 8-\mathrm{C} 7-\mathrm{C} 18$ & $114.09(18)$ & $\mathrm{C} 19-\mathrm{C} 18-\mathrm{C} 7$ & $128.7(2)$ \\
\hline $\mathrm{C} 6-\mathrm{C} 7-\mathrm{C} 18$ & $111.86(19)$ & $\mathrm{C} 18-\mathrm{C} 19-\mathrm{C} 20$ & $117.6(2)$ \\
\hline $\mathrm{C} 13-\mathrm{C} 8-\mathrm{C} 9$ & $116.1(2)$ & $\mathrm{C} 18-\mathrm{C} 19-\mathrm{H} 19$ & $119.1(17)$ \\
\hline $\mathrm{C} 13-\mathrm{C} 8-\mathrm{C} 7$ & $122.29(19)$ & $\mathrm{C} 20-\mathrm{C} 19-\mathrm{H} 19$ & $123.2(17)$ \\
\hline $\mathrm{C} 9-\mathrm{C} 8-\mathrm{C} 7$ & $121.6(2)$ & $\mathrm{C} 19-\mathrm{C} 20-\mathrm{C} 21$ & $121.1(3)$ \\
\hline $\mathrm{C} 10-\mathrm{C} 9-\mathrm{C} 8$ & $122.6(2)$ & $\mathrm{C} 19-\mathrm{C} 20-\mathrm{H} 20$ & $127(2)$ \\
\hline $\mathrm{C} 10-\mathrm{C} 9-\mathrm{H} 9$ & $122.6(15)$ & $\mathrm{C} 21-\mathrm{C} 20-\mathrm{H} 20$ & $112(2)$ \\
\hline $\mathrm{C} 8-\mathrm{C} 9-\mathrm{H} 9$ & $114.8(15)$ & $\mathrm{C} 22-\mathrm{C} 21-\mathrm{C} 20$ & $121.0(2)$ \\
\hline $\mathrm{C} 9-\mathrm{C} 10-\mathrm{C} 11$ & $120.6(2)$ & $\mathrm{C} 22-\mathrm{C} 21-\mathrm{H} 21$ & $120.7(16)$ \\
\hline $\mathrm{C} 9-\mathrm{C} 10-\mathrm{H} 10$ & $118.0(17)$ & $\mathrm{C} 20-\mathrm{C} 21-\mathrm{H} 21$ & $118.3(16)$ \\
\hline $\mathrm{C} 11-\mathrm{C} 10-\mathrm{H} 10$ & $121.4(17)$ & $\mathrm{C} 21-\mathrm{C} 22-\mathrm{C} 23$ & $117.5(2)$ \\
\hline $\mathrm{N} 1-\mathrm{C} 11-\mathrm{C} 12$ & $121.8(2)$ & $\mathrm{C} 21-\mathrm{C} 22-\mathrm{H} 22$ & $123.2(16)$ \\
\hline $\mathrm{N} 1-\mathrm{C} 11-\mathrm{C} 10$ & $121.0(2)$ & $\mathrm{C} 23-\mathrm{C} 22-\mathrm{H} 22$ & $119.3(16)$ \\
\hline
\end{tabular}




\begin{tabular}{|c|c|c|c|}
\hline $\mathrm{C} 12-\mathrm{C} 11-\mathrm{C} 10$ & $117.3(2)$ & $\mathrm{C} 18-\mathrm{C} 23-\mathrm{C} 22$ & $121.6(2)$ \\
\hline $\mathrm{C} 13-\mathrm{C} 12-\mathrm{C} 11$ & $120.1(2)$ & $\mathrm{C} 18-\mathrm{C} 23-\mathrm{C} 24$ & $108.3(2)$ \\
\hline $\mathrm{C} 13-\mathrm{C} 12-\mathrm{H} 12$ & $118.2(16)$ & $\mathrm{C} 22-\mathrm{C} 23-\mathrm{C} 24$ & $130.0(2)$ \\
\hline $\mathrm{C} 11-\mathrm{C} 12-\mathrm{H} 12$ & $121.6(16)$ & $\mathrm{O} 3-\mathrm{C} 24-\mathrm{O} 2$ & $121.5(2)$ \\
\hline $\mathrm{C} 8-\mathrm{C} 13-\mathrm{C} 12$ & $123.2(2)$ & $\mathrm{O} 3-\mathrm{C} 24-\mathrm{C} 23$ & $130.2(2)$ \\
\hline $\mathrm{C} 8-\mathrm{C} 13-\mathrm{O} 1$ & $122.23(18)$ & $\mathrm{O} 2-\mathrm{C} 24-\mathrm{C} 23$ & $108.23(19)$ \\
\hline $\mathrm{C} 13-\mathrm{O} 1-\mathrm{C} 1-\mathrm{C} 6$ & $-7.0(3)$ & $\mathrm{C} 9-\mathrm{C} 10-\mathrm{C} 11-\mathrm{C} 12$ & $-1.2(3)$ \\
\hline $\mathrm{C} 13-\mathrm{O} 1-\mathrm{C} 1-\mathrm{C} 2$ & $172.46(19)$ & $\mathrm{N} 1-\mathrm{C} 11-\mathrm{C} 12-\mathrm{C} 13$ & $-178.9(2)$ \\
\hline $\mathrm{O} 1-\mathrm{C} 1-\mathrm{C} 2-\mathrm{C} 3$ & $-178.0(2)$ & $\mathrm{C} 10-\mathrm{C} 11-\mathrm{C} 12-\mathrm{C} 13$ & $1.2(3)$ \\
\hline $\mathrm{C} 6-\mathrm{C} 1-\mathrm{C} 2-\mathrm{C} 3$ & $1.5(3)$ & $\mathrm{C} 9-\mathrm{C} 8-\mathrm{C} 13-\mathrm{C} 12$ & $0.2(3)$ \\
\hline $\mathrm{C} 1-\mathrm{C} 2-\mathrm{C} 3-\mathrm{C} 4$ & $-1.5(3)$ & $\mathrm{C} 7-\mathrm{C} 8-\mathrm{C} 13-\mathrm{C} 12$ & $-178.6(2)$ \\
\hline $\mathrm{C} 1-\mathrm{C} 2-\mathrm{C} 3-\mathrm{Br} 1$ & $176.79(17)$ & $\mathrm{C} 9-\mathrm{C} 8-\mathrm{C} 13-\mathrm{O} 1$ & $-177.6(2)$ \\
\hline $\mathrm{C} 2-\mathrm{C} 3-\mathrm{C} 4-\mathrm{C} 5$ & $0.5(4)$ & $\mathrm{C} 7-\mathrm{C} 8-\mathrm{C} 13-\mathrm{O} 1$ & $3.6(3)$ \\
\hline $\mathrm{Br} 1-\mathrm{C} 3-\mathrm{C} 4-\mathrm{C} 5$ & $-177.88(18)$ & $\mathrm{C} 11-\mathrm{C} 12-\mathrm{C} 13-\mathrm{C} 8$ & $-0.7(3)$ \\
\hline $\mathrm{C} 3-\mathrm{C} 4-\mathrm{C} 5-\mathrm{C} 6$ & $0.7(4)$ & $\mathrm{C} 11-\mathrm{C} 12-\mathrm{C} 13-\mathrm{O} 1$ & $177.3(2)$ \\
\hline $\mathrm{O} 1-\mathrm{C} 1-\mathrm{C} 6-\mathrm{C} 5$ & $179.1(2)$ & $\mathrm{C} 1-\mathrm{O} 1-\mathrm{C} 13-\mathrm{C} 8$ & $4.4(3)$ \\
\hline $\mathrm{C} 2-\mathrm{C} 1-\mathrm{C} 6-\mathrm{C} 5$ & $-0.4(3)$ & $\mathrm{C} 1-\mathrm{O} 1-\mathrm{C} 13-\mathrm{C} 12$ & $-173.62(18)$ \\
\hline $\mathrm{O} 1-\mathrm{C} 1-\mathrm{C} 6-\mathrm{C} 7$ & $1.7(3)$ & $\mathrm{C} 11-\mathrm{N} 1-\mathrm{C} 14-\mathrm{C} 15$ & $84.3(3)$ \\
\hline $\mathrm{C} 2-\mathrm{C} 1-\mathrm{C} 6-\mathrm{C} 7$ & $-177.8(2)$ & $\mathrm{C} 16-\mathrm{N} 1-\mathrm{C} 14-\mathrm{C} 15$ & $-85.6(3)$ \\
\hline $\mathrm{C} 4-\mathrm{C} 5-\mathrm{C} 6-\mathrm{C} 1$ & $-0.7(4)$ & $\mathrm{C} 11-\mathrm{N} 1-\mathrm{C} 16-\mathrm{C} 17$ & $96.7(3)$ \\
\hline $\mathrm{C} 4-\mathrm{C} 5-\mathrm{C} 6-\mathrm{C} 7$ & $176.7(2)$ & $\mathrm{C} 14-\mathrm{N} 1-\mathrm{C} 16-\mathrm{C} 17$ & $-93.5(3)$ \\
\hline $\mathrm{C} 24-\mathrm{O} 2-\mathrm{C} 7-\mathrm{C} 8$ & $117.59(19)$ & $\mathrm{O} 2-\mathrm{C} 7-\mathrm{C} 18-\mathrm{C} 23$ & $2.5(2)$ \\
\hline $\mathrm{C} 24-\mathrm{O} 2-\mathrm{C} 7-\mathrm{C} 6$ & $-121.50(19)$ & $\mathrm{C} 8-\mathrm{C} 7-\mathrm{C} 18-\mathrm{C} 23$ & $-114.1(2)$ \\
\hline $\mathrm{C} 24-\mathrm{O} 2-\mathrm{C} 7-\mathrm{C} 18$ & $-3.1(2)$ & $\mathrm{C} 6-\mathrm{C} 7-\mathrm{C} 18-\mathrm{C} 23$ & $118.7(2)$ \\
\hline $\mathrm{C} 1-\mathrm{C} 6-\mathrm{C} 7-\mathrm{O} 2$ & $-113.6(2)$ & $\mathrm{O} 2-\mathrm{C} 7-\mathrm{C} 18-\mathrm{C} 19$ & $-175.5(2)$ \\
\hline $\mathrm{C} 5-\mathrm{C} 6-\mathrm{C} 7-\mathrm{O} 2$ & $69.1(3)$ & $\mathrm{C} 8-\mathrm{C} 7-\mathrm{C} 18-\mathrm{C} 19$ & $67.9(3)$ \\
\hline $\mathrm{C} 1-\mathrm{C} 6-\mathrm{C} 7-\mathrm{C} 8$ & $5.6(3)$ & $\mathrm{C} 6-\mathrm{C} 7-\mathrm{C} 18-\mathrm{C} 19$ & $-59.3(3)$ \\
\hline $\mathrm{C} 5-\mathrm{C} 6-\mathrm{C} 7-\mathrm{C} 8$ & $-171.8(2)$ & $\mathrm{C} 23-\mathrm{C} 18-\mathrm{C} 19-\mathrm{C} 20$ & $0.7(3)$ \\
\hline $\mathrm{C} 1-\mathrm{C} 6-\mathrm{C} 7-\mathrm{C} 18$ & $134.4(2)$ & $\mathrm{C} 7-\mathrm{C} 18-\mathrm{C} 19-\mathrm{C} 20$ & $178.5(2)$ \\
\hline $\mathrm{C} 5-\mathrm{C} 6-\mathrm{C} 7-\mathrm{C} 18$ & $-43.0(3)$ & $\mathrm{C} 18-\mathrm{C} 19-\mathrm{C} 20-\mathrm{C} 21$ & $-1.0(4)$ \\
\hline $\mathrm{O} 2-\mathrm{C} 7-\mathrm{C} 8-\mathrm{C} 13$ & $111.4(2)$ & $\mathrm{C} 19-\mathrm{C} 20-\mathrm{C} 21-\mathrm{C} 22$ & $0.6(4)$ \\
\hline $\mathrm{C} 6-\mathrm{C} 7-\mathrm{C} 8-\mathrm{C} 13$ & $-8.1(3)$ & $\mathrm{C} 20-\mathrm{C} 21-\mathrm{C} 22-\mathrm{C} 23$ & $0.0(4)$ \\
\hline $\mathrm{C} 18-\mathrm{C} 7-\mathrm{C} 8-\mathrm{C} 13$ & $-135.7(2)$ & $\mathrm{C} 19-\mathrm{C} 18-\mathrm{C} 23-\mathrm{C} 22$ & $-0.1(4)$ \\
\hline $\mathrm{O} 2-\mathrm{C} 7-\mathrm{C} 8-\mathrm{C} 9$ & $-67.3(3)$ & $\mathrm{C} 7-\mathrm{C} 18-\mathrm{C} 23-\mathrm{C} 22$ & $-178.3(2)$ \\
\hline $\mathrm{C} 6-\mathrm{C} 7-\mathrm{C} 8-\mathrm{C} 9$ & $173.2(2)$ & $\mathrm{C} 19-\mathrm{C} 18-\mathrm{C} 23-\mathrm{C} 24$ & $177.1(2)$ \\
\hline $\mathrm{C} 18-\mathrm{C} 7-\mathrm{C} 8-\mathrm{C} 9$ & $45.6(3)$ & $\mathrm{C} 7-\mathrm{C} 18-\mathrm{C} 23-\mathrm{C} 24$ & $-1.1(3)$ \\
\hline $\mathrm{C} 13-\mathrm{C} 8-\mathrm{C} 9-\mathrm{C} 10$ & $-0.2(3)$ & $\mathrm{C} 21-\mathrm{C} 22-\mathrm{C} 23-\mathrm{C} 18$ & $-0.3(4)$ \\
\hline $\mathrm{C} 7-\mathrm{C} 8-\mathrm{C} 9-\mathrm{C} 10$ & $178.6(2)$ & $\mathrm{C} 21-\mathrm{C} 22-\mathrm{C} 23-\mathrm{C} 24$ & $-176.8(2)$ \\
\hline $\mathrm{C} 8-\mathrm{C} 9-\mathrm{C} 10-\mathrm{C} 11$ & $0.8(4)$ & $\mathrm{C} 7-\mathrm{O} 2-\mathrm{C} 24-\mathrm{O} 3$ & $-177.8(2)$ \\
\hline $\mathrm{C} 14-\mathrm{N} 1-\mathrm{C} 11-\mathrm{C} 12$ & $-1.9(3)$ & $\mathrm{C} 7-\mathrm{O} 2-\mathrm{C} 24-\mathrm{C} 23$ & $2.6(2)$ \\
\hline $\mathrm{C} 16-\mathrm{N} 1-\mathrm{C} 11-\mathrm{C} 12$ & $167.3(2)$ & $\mathrm{C} 18-\mathrm{C} 23-\mathrm{C} 24-\mathrm{O} 3$ & $179.5(2)$ \\
\hline $\mathrm{C} 14-\mathrm{N} 1-\mathrm{C} 11-\mathrm{C} 10$ & $178.0(2)$ & $\mathrm{C} 22-\mathrm{C} 23-\mathrm{C} 24-\mathrm{O} 3$ & $-3.6(4)$ \\
\hline $\mathrm{C} 16-\mathrm{N} 1-\mathrm{C} 11-\mathrm{C} 10$ & $-12.9(4)$ & $\mathrm{C} 18-\mathrm{C} 23-\mathrm{C} 24-\mathrm{O} 2$ & $-0.9(3)$ \\
\hline $\mathrm{C} 9-\mathrm{C} 10-\mathrm{C} 11-\mathrm{N} 1$ & $178.9(2)$ & $\mathrm{C} 22-\mathrm{C} 23-\mathrm{C} 24-\mathrm{O} 2$ & $176.0(2)$ \\
\hline
\end{tabular}


Hydrogen-bond geometry $\left(\AA,{ }^{\circ}\right)$

\begin{tabular}{lllll}
\hline$D-\mathrm{H} \cdots A$ & $D-\mathrm{H}$ & $\mathrm{H} \cdots A$ & $D \cdots A$ & $D-\mathrm{H} \cdots A$ \\
\hline $\mathrm{C} 14-\mathrm{H} 14 B \cdots \mathrm{O} 3^{\mathrm{i}}$ & $0.99(2)$ & $2.68(2)$ & $3.621(3)$ & $160.7(19)$ \\
$\mathrm{C} 20-\mathrm{H} 20 \cdots 3^{\mathrm{ii}}$ & $0.94(2)$ & $2.41(3)$ & $3.163(3)$ & $138(3)$ \\
\hline
\end{tabular}

Symmetry codes: (i) $-x+2, y+1 / 2,-z+1 / 2$; (ii) $-x+2, y+1 / 2,-z+3 / 2$.

(5) (1R)-6'-Bromo-3'-diethylamino-3H-spiro[2-benzofuran-1,9'-xanthen]-3-one

Crystal data

$\mathrm{C}_{24} \mathrm{H}_{20} \mathrm{BrNO}_{3}$

$M_{r}=450.32$

Orthorhombic, $P 22_{1} 2_{1}$

$a=8.1529$ (13) $\AA$

$b=18.185(3) \AA$

$c=26.860(4) \AA$

$V=3982.3(11) \AA^{3}$

$Z=8$

$F(000)=1840$

Data collection

Bruker SMART APEX CCD

diffractometer

Radiation source: fine-focus sealed tube

Graphite monochromator

Detector resolution: 8.3333 pixels $\mathrm{mm}^{-1}$

$\varphi$ and $\omega$ scans

Absorption correction: multi-scan

(SADABS; Bruker, 2016)

$T_{\min }=0.70, T_{\max }=0.92$

Refinement

Refinement on $F^{2}$

Least-squares matrix: full

$R\left[F^{2}>2 \sigma\left(F^{2}\right)\right]=0.045$

$w R\left(F^{2}\right)=0.093$

$S=0.97$

10174 reflections

527 parameters

0 restraints

Primary atom site location: structure-invariant direct methods

Secondary atom site location: difference Fourier map
$D_{\mathrm{x}}=1.502 \mathrm{Mg} \mathrm{m}^{-3}$

Mo $K \alpha$ radiation, $\lambda=0.71073 \AA$

Cell parameters from 7498 reflections

$\theta=2.2-25.3^{\circ}$

$\mu=2.09 \mathrm{~mm}^{-1}$

$T=100 \mathrm{~K}$

Column, colourless

$0.26 \times 0.06 \times 0.04 \mathrm{~mm}$

38240 measured reflections

10174 independent reflections

7285 reflections with $I>2 \sigma(I)$

$R_{\text {int }}=0.075$

$\theta_{\max }=29.1^{\circ}, \theta_{\min }=1.4^{\circ}$

$h=-10 \rightarrow 10$

$k=-23 \rightarrow 24$

$l=-35 \rightarrow 36$

Hydrogen site location: inferred from neighbouring sites

$\mathrm{H}$-atom parameters constrained

$w=1 /\left[\sigma^{2}\left(F_{\mathrm{o}}^{2}\right)+(0.0089 P)^{2}\right]$

where $P=\left(F_{\mathrm{o}}^{2}+2 F_{\mathrm{c}}^{2}\right) / 3$

$(\Delta / \sigma)_{\max }=0.001$

$\Delta \rho_{\max }=0.92 \mathrm{e} \AA^{-3}$

$\Delta \rho_{\min }=-0.34$ e $\AA^{-3}$

Absolute structure: Flack $x$ determined using 2575 quotients $\left[\left(I^{+}\right)-(I)\right] /\left[\left(I^{+}\right)+\left(I^{-}\right)\right.$(Parsons et al., 2013)

Absolute structure parameter: $-0.002(6)$

\section{Special details}

Experimental. The diffraction data were collected in three sets of 363 frames $\left(0.5^{\circ}\right.$ width in $\left.\omega\right)$ at $\varphi=0,120$ and $240^{\circ}$. A scan time of $60 \mathrm{sec} /$ frame was used.

Geometry. All esds (except the esd in the dihedral angle between two 1.s. planes) are estimated using the full covariance matrix. The cell esds are taken into account individually in the estimation of esds in distances, angles and torsion angles; correlations between esds in cell parameters are only used when they are defined by crystal symmetry. An approximate (isotropic) treatment of cell esds is used for estimating esds involving l.s. planes. 
Refinement. Refinement of $\mathrm{F}^{2}$ against ALL reflections. The weighted R-factor $\mathrm{wR}$ and goodness of fit $\mathrm{S}$ are based on $\mathrm{F}^{2}$, conventional R-factors $R$ are based on $F$, with $F$ set to zero for negative $F^{2}$. The threshold expression of $F^{2}>2 \operatorname{sigma}\left(F^{2}\right)$ is used only for calculating R-factors(gt) etc. and is not relevant to the choice of reflections for refinement. R-factors based on $\mathrm{F}^{2}$ are statistically about twice as large as those based on F, and R- factors based on ALL data will be even larger. Hatoms attached to carbon were placed in calculated positions $(\mathrm{C}-\mathrm{H}=0.95-0.99 \AA)$. All were included as riding contributions with isotropic displacement parameters $1.2-1.5$ times those of the attached atoms.

Fractional atomic coordinates and isotropic or equivalent isotropic displacement parameters $\left(\AA^{2}\right)$

\begin{tabular}{|c|c|c|c|c|}
\hline & $x$ & $y$ & $z$ & $U_{\text {iso }} * / U_{\text {eq }}$ \\
\hline $\mathrm{Br} 1$ & $0.52585(6)$ & $0.63496(3)$ & $0.28218(2)$ & $0.02849(13)$ \\
\hline $\mathrm{O} 1$ & $0.6662(4)$ & $0.57150(16)$ & $0.46407(11)$ & $0.0189(7)$ \\
\hline $\mathrm{O} 2$ & 0.4245 & $0.39341(16)$ & $0.46124(11)$ & $0.0153(7)$ \\
\hline $\mathrm{O} 3$ & $0.2630(4)$ & $0.29559(18)$ & $0.44638(11)$ & $0.0207(7)$ \\
\hline N1 & $0.8582(5)$ & $0.5506(2)$ & $0.63051(13)$ & $0.0178(8)$ \\
\hline $\mathrm{C} 1$ & $0.6260(5)$ & $0.5413(2)$ & $0.41884(16)$ & $0.0160(10)$ \\
\hline $\mathrm{C} 2$ & $0.6056(5)$ & $0.5918(2)$ & $0.38032(16)$ & $0.0181(10)$ \\
\hline $\mathrm{H} 2$ & 0.6212 & 0.6429 & 0.3860 & $0.022 *$ \\
\hline $\mathrm{C} 3$ & $0.5626(5)$ & $0.5663(3)$ & $0.33398(16)$ & $0.0191(10)$ \\
\hline $\mathrm{C} 4$ & $0.5443(5)$ & $0.4916(3)$ & $0.32441(16)$ & $0.0199(10)$ \\
\hline $\mathrm{H} 4$ & 0.5175 & 0.4747 & 0.2920 & $0.024 *$ \\
\hline $\mathrm{C} 5$ & $0.5659(5)$ & $0.4425(2)$ & $0.36318(16)$ & $0.0180(10)$ \\
\hline H5 & 0.5552 & 0.3913 & 0.3571 & $0.022 *$ \\
\hline C6 & $0.6035(5)$ & $0.4671(2)$ & $0.41148(16)$ & $0.0149(9)$ \\
\hline $\mathrm{C} 7$ & $0.6029(5)$ & $0.4136(2)$ & $0.45477(15)$ & $0.0136(9)$ \\
\hline $\mathrm{C} 8$ & $0.6652(5)$ & $0.4501(2)$ & $0.50108(15)$ & $0.0130(9)$ \\
\hline $\mathrm{C} 9$ & $0.6964(5)$ & $0.4098(2)$ & $0.54461(16)$ & $0.0157(10)$ \\
\hline H9 & 0.6778 & 0.3583 & 0.5443 & $0.019 *$ \\
\hline $\mathrm{C} 10$ & $0.7528(5)$ & $0.4418(2)$ & $0.58754(16)$ & $0.0137(9)$ \\
\hline H10 & 0.7699 & 0.4124 & 0.6163 & $0.016^{*}$ \\
\hline C11 & $0.7859(5)$ & $0.5186(2)$ & $0.58949(16)$ & $0.0152(9)$ \\
\hline $\mathrm{C} 12$ & $0.7507(5)$ & $0.5598(2)$ & $0.54662(16)$ & $0.0156(10)$ \\
\hline H12 & 0.7657 & 0.6116 & 0.5467 & $0.019 *$ \\
\hline $\mathrm{C} 13$ & $0.6939(5)$ & $0.5246(2)$ & $0.50400(16)$ & $0.0147(9)$ \\
\hline $\mathrm{C} 14$ & $0.8803(6)$ & $0.5091(3)$ & $0.67630(16)$ & $0.0214(11)$ \\
\hline $\mathrm{H} 14 \mathrm{~A}$ & 0.9644 & 0.5337 & 0.6970 & $0.026^{*}$ \\
\hline H14B & 0.9215 & 0.4594 & 0.6680 & $0.026^{*}$ \\
\hline C15 & $0.7228(6)$ & $0.5018(3)$ & $0.70632(17)$ & $0.0221(11)$ \\
\hline $\mathrm{H} 15 \mathrm{~A}$ & 0.6887 & 0.5504 & 0.7182 & $0.033 *$ \\
\hline H15B & 0.7420 & 0.4693 & 0.7349 & $0.033^{*}$ \\
\hline $\mathrm{H} 15 \mathrm{C}$ & 0.6365 & 0.4809 & 0.6852 & $0.033 *$ \\
\hline $\mathrm{C} 16$ & $0.8935(5)$ & $0.6297(3)$ & $0.63138(17)$ & $0.0219(10)$ \\
\hline H16A & 0.9442 & 0.6437 & 0.5993 & $0.026^{*}$ \\
\hline H16B & 0.9746 & 0.6396 & 0.6580 & $0.026^{*}$ \\
\hline $\mathrm{C} 17$ & $0.7432(6)$ & 0.6778 & $0.64011(19)$ & $0.0303(13)$ \\
\hline H17A & 0.7756 & 0.7297 & 0.6393 & $0.045^{*}$ \\
\hline H17B & 0.6955 & 0.6663 & 0.6727 & $0.045^{*}$ \\
\hline $\mathrm{H} 17 \mathrm{C}$ & 0.6620 & 0.6684 & 0.6140 & $0.045^{*}$ \\
\hline
\end{tabular}




\begin{tabular}{|c|c|c|c|c|}
\hline $\mathrm{C} 18$ & $0.6792(5)$ & $0.3401(2)$ & $0.44466(15)$ & $0.0131(9)$ \\
\hline C19 & $0.8454(5)$ & $0.3221(2)$ & $0.44054(16)$ & $0.0153(10)$ \\
\hline H19 & 0.9282 & 0.3586 & 0.4430 & $0.018^{*}$ \\
\hline $\mathrm{C} 20$ & $0.8845(6)$ & $0.2494(2)$ & $0.43276(15)$ & $0.0179(10)$ \\
\hline $\mathrm{H} 20$ & 0.9964 & 0.2357 & 0.4295 & $0.022 *$ \\
\hline $\mathrm{C} 21$ & $0.7630(6)$ & $0.1948(2)$ & $0.42956(16)$ & $0.0174(10)$ \\
\hline $\mathrm{H} 21$ & 0.7943 & 0.1451 & 0.4244 & $0.021 *$ \\
\hline $\mathrm{C} 22$ & $0.5991(6)$ & $0.2123(2)$ & $0.43380(15)$ & $0.0163(10)$ \\
\hline $\mathrm{H} 22$ & 0.5162 & 0.1757 & 0.4315 & $0.020 *$ \\
\hline $\mathrm{C} 23$ & $0.5604(5)$ & $0.2865(2)$ & $0.44160(15)$ & $0.0132(9)$ \\
\hline $\mathrm{C} 24$ & $0.3991(6)$ & $0.3206(2)$ & $0.44915(15)$ & $0.0146(9)$ \\
\hline $\mathrm{Br} 2$ & $0.41662(7)$ & $0.36508(3)$ & $0.88995(2)$ & $0.03217(14)$ \\
\hline $\mathrm{O} 4$ & $0.3369(4)$ & $0.51090(15)$ & $0.72672(11)$ & $0.0169(7)$ \\
\hline O5 & $0.2069(3)$ & $0.69908(16)$ & $0.79230(11)$ & $0.0160(7)$ \\
\hline O6 & $0.1534(4)$ & $0.80937(16)$ & $0.82613(12)$ & $0.0197(7)$ \\
\hline N2 & $0.2868(5)$ & $0.61160(19)$ & $0.56403(13)$ & $0.0177(9)$ \\
\hline $\mathrm{C} 25$ & $0.3579(5)$ & $0.5173(2)$ & $0.77747(15)$ & $0.0138(9)$ \\
\hline $\mathrm{C} 26$ & $0.3746(5)$ & $0.4512(2)$ & $0.80330(16)$ & $0.0181(10)$ \\
\hline $\mathrm{H} 26$ & 0.3753 & 0.4054 & 0.7863 & $0.022 *$ \\
\hline $\mathrm{C} 27$ & $0.3900(6)$ & $0.4545(2)$ & $0.85438(17)$ & $0.0216(11)$ \\
\hline $\mathrm{C} 28$ & $0.3868(6)$ & $0.5198(2)$ & $0.88052(17)$ & $0.0210(11)$ \\
\hline $\mathrm{H} 28$ & 0.3940 & 0.5202 & 0.9158 & $0.025^{*}$ \\
\hline $\mathrm{C} 29$ & $0.3727(5)$ & $0.5850(3)$ & $0.85389(16)$ & $0.0188(10)$ \\
\hline $\mathrm{H} 29$ & 0.3722 & 0.6305 & 0.8713 & $0.023 *$ \\
\hline $\mathrm{C} 30$ & $0.3594(5)$ & $0.5848(2)$ & $0.80207(16)$ & $0.0147(9)$ \\
\hline $\mathrm{C} 31$ & $0.3510(5)$ & $0.6566(2)$ & $0.77414(16)$ & $0.0149(9)$ \\
\hline $\mathrm{C} 32$ & $0.3316(5)$ & $0.6439(2)$ & $0.71915(16)$ & $0.0134(9)$ \\
\hline $\mathrm{C} 33$ & $0.3218(5)$ & $0.7029(2)$ & $0.68601(17)$ & $0.0167(10)$ \\
\hline $\mathrm{H} 33$ & 0.3261 & 0.7515 & 0.6988 & $0.020 *$ \\
\hline C34 & $0.3063(5)$ & $0.6929(2)$ & $0.63567(17)$ & $0.0157(10)$ \\
\hline H34 & 0.2980 & 0.7347 & 0.6146 & $0.019 *$ \\
\hline $\mathrm{C} 35$ & $0.3021(5)$ & $0.6217(2)$ & $0.61428(16)$ & $0.0140(9)$ \\
\hline $\mathrm{C} 36$ & $0.3144(5)$ & $0.5622(2)$ & $0.64719(16)$ & $0.0155(10)$ \\
\hline H36 & 0.3142 & 0.5134 & 0.6346 & $0.019^{*}$ \\
\hline $\mathrm{C} 37$ & $0.3271(5)$ & $0.5742(2)$ & $0.69832(17)$ & $0.0153(10)$ \\
\hline C38 & $0.2429(6)$ & $0.6721(3)$ & $0.53094(16)$ & $0.0206(11)$ \\
\hline $\mathrm{H} 38 \mathrm{~A}$ & 0.1740 & 0.7075 & 0.5495 & $0.025^{*}$ \\
\hline H38B & 0.1762 & 0.6524 & 0.5032 & $0.025^{*}$ \\
\hline C39 & $0.3903(7)$ & $0.7128(3)$ & 0.50945 (19) & $0.0316(13)$ \\
\hline H39A & 0.4631 & 0.6776 & 0.4928 & $0.047^{*}$ \\
\hline H39B & 0.4499 & 0.7373 & 0.5364 & $0.047^{*}$ \\
\hline $\mathrm{H} 39 \mathrm{C}$ & 0.3525 & 0.7495 & 0.4853 & $0.047^{*}$ \\
\hline $\mathrm{C} 40$ & $0.3094(5)$ & $0.5399(2)$ & $0.54013(16)$ & $0.0157(10)$ \\
\hline $\mathrm{H} 40 \mathrm{~A}$ & 0.3904 & 0.5113 & 0.5596 & $0.019^{*}$ \\
\hline $\mathrm{H} 40 \mathrm{~B}$ & 0.3559 & 0.5477 & 0.5065 & $0.019^{*}$ \\
\hline $\mathrm{C} 41$ & $0.1539(6)$ & $0.4950(3)$ & $0.53531(18)$ & $0.0235(11)$ \\
\hline $\mathrm{H} 41 \mathrm{~A}$ & 0.0719 & 0.5232 & 0.5167 & $0.035^{*}$ \\
\hline H41B & 0.1113 & 0.4835 & 0.5685 & $0.035^{*}$ \\
\hline
\end{tabular}




$\begin{array}{lllll}\mathrm{H} 41 \mathrm{C} & 0.1780 & 0.4491 & 0.5176 & 0.035^{*} \\ \mathrm{C} 42 & 0.4921(5) & 0.7074(2) & 0.78662(15) & 0.0146(9) \\ \mathrm{C} 43 & 0.6590(5) & 0.6963(2) & 0.78122(17) & 0.0173(10) \\ \mathrm{H} 43 & 0.7009 & 0.6508 & 0.7692 & 0.021^{*} \\ \mathrm{C} 44 & 0.7637(6) & 0.7538(3) & 0.79392(16) & 0.0202(11) \\ \mathrm{H} 44 & 0.8789 & 0.7471 & 0.7912 & 0.024^{*} \\ \mathrm{C} 45 & 0.7029(6) & 0.8208(3) & 0.81042(16) & 0.0188(10) \\ \mathrm{H} 45 & 0.7765 & 0.8601 & 0.8171 & 0.023^{*} \\ \text { C46 } & 0.5359(6) & 0.8311(2) & 0.81717(15) & 0.0171(10) \\ \text { H46 } & 0.4936 & 0.8761 & 0.8296 & 0.021^{*} \\ \text { C47 } & 0.4327(5) & 0.7732(2) & 0.80510(15) & 0.0140(10) \\ \text { C48 } & 0.2526(6) & 0.7669(2) & 0.80989(15) & 0.0143(9)\end{array}$

Atomic displacement parameters $\left(\AA^{2}\right)$

\begin{tabular}{|c|c|c|c|c|c|c|}
\hline & $U^{11}$ & $U^{22}$ & $U^{33}$ & $U^{12}$ & $U^{13}$ & $U^{23}$ \\
\hline $\mathrm{Br} 1$ & $0.0362(3)$ & $0.0288(3)$ & $0.0205(2)$ & $-0.0066(2)$ & $-0.0075(2)$ & $0.0118(2)$ \\
\hline $\mathrm{O} 1$ & $0.0273(19)$ & $0.0155(17)$ & $0.0138(16)$ & $-0.0017(14)$ & $-0.0018(14)$ & $0.0019(13)$ \\
\hline $\mathrm{O} 2$ & $0.0097(16)$ & $0.0177(15)$ & $0.0185(16)$ & $0.0001(12)$ & $0.0024(13)$ & $0.0030(12)$ \\
\hline $\mathrm{O} 3$ & $0.0149(18)$ & $0.028(2)$ & $0.0191(18)$ & $-0.0049(15)$ & -0.0008 (14) & $0.0029(14)$ \\
\hline N1 & $0.018(2)$ & $0.021(2)$ & $0.0152(19)$ & $0.0014(17)$ & $-0.0011(16)$ & $0.0002(16)$ \\
\hline $\mathrm{C} 1$ & $0.013(2)$ & $0.020(2)$ & $0.015(2)$ & $0.0021(19)$ & $-0.0005(18)$ & $-0.0004(18)$ \\
\hline $\mathrm{C} 2$ & $0.019(3)$ & $0.014(2)$ & $0.021(2)$ & $0.000(2)$ & $-0.002(2)$ & $0.0045(18)$ \\
\hline $\mathrm{C} 3$ & $0.016(3)$ & 0.024 & $0.017(2)$ & $-0.002(2)$ & $0.0003(19)$ & $0.0095(19)$ \\
\hline $\mathrm{C} 4$ & $0.019(3)$ & $0.027(3)$ & $0.014(2)$ & $-0.004(2)$ & -0.0009 (19) & $0.0027(19)$ \\
\hline $\mathrm{C} 5$ & $0.017(3)$ & $0.016(2)$ & $0.022(2)$ & $-0.0027(19)$ & $0.0017(19)$ & $0.0015(19)$ \\
\hline C6 & $0.010(2)$ & $0.015(2)$ & $0.019(2)$ & $0.0011(19)$ & $0.0011(19)$ & $0.0013(18)$ \\
\hline $\mathrm{C} 7$ & $0.010(2)$ & $0.014(2)$ & $0.017(2)$ & $-0.0022(19)$ & $0.0011(18)$ & $0.0006(18)$ \\
\hline $\mathrm{C} 8$ & $0.011(2)$ & $0.016(2)$ & $0.012(2)$ & $0.0033(18)$ & $0.0044(18)$ & $0.0017(18)$ \\
\hline C9 & $0.015(2)$ & $0.011(2)$ & $0.021(2)$ & $0.0030(19)$ & $0.0031(19)$ & $0.0022(18)$ \\
\hline $\mathrm{C} 10$ & $0.015(2)$ & $0.014(2)$ & $0.012(2)$ & $0.0042(19)$ & $0.0017(18)$ & $0.0010(17)$ \\
\hline C11 & $0.010(2)$ & $0.018(2)$ & $0.018(2)$ & $0.0016(19)$ & $0.0016(19)$ & 0.0004 (19) \\
\hline $\mathrm{C} 12$ & $0.013(2)$ & $0.013(2)$ & $0.021(2)$ & $0.0006(18)$ & $0.0000(19)$ & $0.0001(19)$ \\
\hline $\mathrm{C} 13$ & $0.013(2)$ & $0.016(2)$ & $0.015(2)$ & $0.0046(18)$ & $0.0008(18)$ & $0.0054(18)$ \\
\hline $\mathrm{C} 14$ & $0.023(3)$ & $0.024(3)$ & $0.017(2)$ & $-0.001(2)$ & $-0.006(2)$ & $0.000(2)$ \\
\hline $\mathrm{C} 15$ & $0.025(3)$ & $0.025(3)$ & $0.016(2)$ & $-0.003(2)$ & $0.001(2)$ & $0.000(2)$ \\
\hline $\mathrm{C} 16$ & $0.022(2)$ & $0.021(2)$ & $0.023(2)$ & $0.000(2)$ & $0.0003(19)$ & $-0.001(2)$ \\
\hline $\mathrm{C} 17$ & $0.035(3)$ & $0.026(3)$ & $0.030(3)$ & $0.008(2)$ & $-0.001(2)$ & $-0.002(2)$ \\
\hline $\mathrm{C} 18$ & $0.016(2)$ & $0.015(2)$ & $0.009(2)$ & $0.0005(18)$ & $-0.0009(17)$ & $0.0006(17)$ \\
\hline C19 & $0.013(2)$ & $0.018(2)$ & $0.014(2)$ & $-0.0031(19)$ & $-0.0002(18)$ & $-0.0012(18)$ \\
\hline $\mathrm{C} 20$ & $0.016(3)$ & $0.023(3)$ & $0.015(2)$ & $0.002(2)$ & $0.0020(19)$ & $-0.0012(19)$ \\
\hline $\mathrm{C} 21$ & $0.025(3)$ & $0.010(2)$ & $0.018(2)$ & $0.006(2)$ & $0.002(2)$ & $-0.0019(18)$ \\
\hline $\mathrm{C} 22$ & $0.017(2)$ & $0.016(2)$ & $0.016(2)$ & $-0.007(2)$ & -0.0005 (19) & $-0.0013(18)$ \\
\hline $\mathrm{C} 23$ & $0.010(2)$ & $0.018(2)$ & $0.011(2)$ & $-0.0027(18)$ & $-0.0023(17)$ & $0.0008(17)$ \\
\hline $\mathrm{C} 24$ & $0.019(2)$ & $0.017(2)$ & $0.009(2)$ & $-0.002(2)$ & $-0.0042(19)$ & $0.0048(17)$ \\
\hline $\mathrm{Br} 2$ & $0.0550(4)$ & $0.0189(2)$ & $0.0226(3)$ & $-0.0016(3)$ & $-0.0038(2)$ & $0.0063(2)$ \\
\hline $\mathrm{O} 4$ & $0.0253(18)$ & $0.0118(16)$ & $0.0137(16)$ & $0.0004(13)$ & $-0.0002(13)$ & $-0.0007(12)$ \\
\hline $\mathrm{O} 5$ & $0.0130(16)$ & $0.0137(16)$ & $0.0214(18)$ & 0.0008 (13) & $0.0012(13)$ & $-0.0047(13)$ \\
\hline
\end{tabular}




\begin{tabular}{|c|c|c|c|c|c|c|}
\hline O6 & $0.0212(18)$ & $0.0146(17)$ & $0.0234(18)$ & $0.0058(14)$ & $-0.0011(14)$ & $-0.0038(13)$ \\
\hline $\mathrm{N} 2$ & $0.025(2)$ & $0.0130(19)$ & $0.0146(19)$ & $0.0028(16)$ & $0.0010(17)$ & $0.0005(15)$ \\
\hline $\mathrm{C} 25$ & $0.011(2)$ & $0.019(2)$ & $0.011(2)$ & $-0.0026(18)$ & $0.0023(18)$ & $-0.0015(18)$ \\
\hline $\mathrm{C} 26$ & $0.023(3)$ & $0.013(2)$ & $0.018(2)$ & $-0.002(2)$ & $-0.001(2)$ & $0.0002(18)$ \\
\hline $\mathrm{C} 27$ & $0.024(3)$ & $0.016(2)$ & $0.025(3)$ & $-0.001(2)$ & $-0.005(2)$ & $0.002(2)$ \\
\hline $\mathrm{C} 28$ & $0.030(3)$ & $0.017(2)$ & $0.016(2)$ & $-0.001(2)$ & $-0.003(2)$ & $0.0015(18)$ \\
\hline $\mathrm{C} 29$ & $0.023(3)$ & $0.017(2)$ & $0.017(2)$ & $-0.003(2)$ & $-0.002(2)$ & $-0.0030(19)$ \\
\hline $\mathrm{C} 30$ & $0.012(2)$ & $0.016(2)$ & $0.017(2)$ & -0.0029 (19) & $-0.0013(18)$ & $-0.0002(18)$ \\
\hline C31 & $0.011(2)$ & $0.015(2)$ & $0.019(2)$ & $0.0036(17)$ & $0.0035(18)$ & $-0.0014(18)$ \\
\hline C32 & $0.013(2)$ & $0.012(2)$ & $0.016(2)$ & $-0.0023(18)$ & $-0.0001(18)$ & $0.0016(19)$ \\
\hline $\mathrm{C} 33$ & $0.014(2)$ & $0.014(2)$ & $0.022(3)$ & $0.0023(19)$ & $0.002(2)$ & $0.0011(19)$ \\
\hline $\mathrm{C} 34$ & $0.014(2)$ & $0.011(2)$ & $0.022(2)$ & $0.0005(19)$ & $-0.0011(19)$ & $0.0038(19)$ \\
\hline $\mathrm{C} 35$ & $0.011(2)$ & $0.016(2)$ & $0.015(2)$ & $0.0015(17)$ & $0.0013(17)$ & $-0.0025(18)$ \\
\hline C36 & $0.015(2)$ & $0.013(2)$ & $0.019(2)$ & $0.0032(19)$ & 0.0007 (19) & $-0.0016(19)$ \\
\hline $\mathrm{C} 37$ & $0.011(2)$ & $0.015(2)$ & $0.020(2)$ & $-0.0014(19)$ & $0.0010(18)$ & $0.0013(19)$ \\
\hline $\mathrm{C} 38$ & $0.027(3)$ & $0.021(3)$ & $0.014(2)$ & $0.004(2)$ & $-0.003(2)$ & $0.0025(19)$ \\
\hline C39 & $0.040(3)$ & 0.023 & $0.032(3)$ & $0.001(3)$ & $0.011(3)$ & $0.006(2)$ \\
\hline $\mathrm{C} 40$ & $0.014(2)$ & $0.020(2)$ & $0.013(2)$ & $0.004(2)$ & $-0.0001(18)$ & $-0.0015(18)$ \\
\hline $\mathrm{C} 41$ & $0.019(3)$ & $0.024(3)$ & $0.027(3)$ & $0.001(2)$ & $0.001(2)$ & $-0.004(2)$ \\
\hline $\mathrm{C} 42$ & $0.018(2)$ & $0.012(2)$ & $0.014(2)$ & $-0.0009(18)$ & $-0.0014(19)$ & $-0.0008(18)$ \\
\hline $\mathrm{C} 43$ & $0.017(2)$ & $0.020(2)$ & $0.015(2)$ & $0.0016(19)$ & $0.000(2)$ & $-0.001(2)$ \\
\hline $\mathrm{C} 44$ & $0.017(3)$ & 0.025 & $0.019(3)$ & $-0.002(2)$ & -0.0004 (19) & $0.003(2)$ \\
\hline $\mathrm{C} 45$ & $0.020(3)$ & $0.021(3)$ & $0.016(2)$ & $-0.007(2)$ & $-0.0051(19)$ & $0.0021(19)$ \\
\hline $\mathrm{C} 46$ & $0.026(3)$ & $0.013(2)$ & $0.013(2)$ & $-0.001(2)$ & $-0.002(2)$ & $0.0020(18)$ \\
\hline $\mathrm{C} 47$ & $0.018(3)$ & $0.015(2)$ & $0.009(2)$ & $-0.0012(19)$ & $-0.0036(18)$ & $0.0028(17)$ \\
\hline $\mathrm{C} 48$ & $0.019(2)$ & $0.016(2)$ & $0.008(2)$ & $0.004(2)$ & $-0.0014(18)$ & $0.0019(18)$ \\
\hline
\end{tabular}

Geometric parameters $\left(\AA,{ }^{\circ}\right)$

\begin{tabular}{llll}
\hline $\mathrm{Br} 1-\mathrm{C} 3$ & $1.893(4)$ & $\mathrm{Br} 2-\mathrm{C} 27$ & $1.899(4)$ \\
$\mathrm{O} 1-\mathrm{C} 1$ & $1.373(5)$ & $\mathrm{O} 4-\mathrm{C} 25$ & $1.379(5)$ \\
$\mathrm{O} 1-\mathrm{C} 13$ & $1.389(5)$ & $\mathrm{O} 4-\mathrm{C} 37$ & $1.383(5)$ \\
$\mathrm{O} 2-\mathrm{C} 24$ & $1.379(5)$ & $\mathrm{O} 5-\mathrm{C} 48$ & $1.373(5)$ \\
$\mathrm{O} 2-\mathrm{C} 7$ & $\mathrm{O} 5-\mathrm{C} 31$ & $1.488(5)$ \\
$\mathrm{O} 3-\mathrm{C} 24$ & $1.510(5)$ & $\mathrm{O} 6-\mathrm{C} 48$ & $1.200(5)$ \\
$\mathrm{N} 1-\mathrm{C} 11$ & $1.201(5)$ & $\mathrm{N} 2-\mathrm{C} 35$ & $1.368(5)$ \\
$\mathrm{N} 1-\mathrm{C} 14$ & $1.379(5)$ & $\mathrm{N} 2-\mathrm{C} 38$ & $1.458(5)$ \\
$\mathrm{N} 1-\mathrm{C} 16$ & $1.455(5)$ & $\mathrm{N} 2-\mathrm{C} 40$ & $1.465(5)$ \\
$\mathrm{C} 1-\mathrm{C} 6$ & $1.466(6)$ & $\mathrm{C} 25-\mathrm{C} 26$ & $1.394(6)$ \\
$\mathrm{C} 1-\mathrm{C} 2$ & $1.376(6)$ & $\mathrm{C} 25-\mathrm{C} 30$ & $1.394(6)$ \\
$\mathrm{C} 2-\mathrm{C} 3$ & $1.394(6)$ & $\mathrm{C} 26-\mathrm{C} 27$ & $1.379(6)$ \\
$\mathrm{C} 2-\mathrm{H} 2$ & $1.374(6)$ & $\mathrm{C} 26-\mathrm{H} 26$ & 0.9500 \\
$\mathrm{C} 3-\mathrm{C} 4$ & 0.9500 & $\mathrm{C} 27-\mathrm{C} 28$ & $1.380(6)$ \\
$\mathrm{C} 4-\mathrm{C} 5$ & $1.390(6)$ & $\mathrm{C} 28-\mathrm{C} 29$ & $1.388(6)$ \\
$\mathrm{C} 4-\mathrm{H} 4$ & $1.383(6)$ & $\mathrm{C} 29-\mathrm{H} 28$ & 0.9500 \\
$\mathrm{C} 5-\mathrm{C} 6$ & 0.9500 & $\mathrm{C} 29-\mathrm{H} 29$ & $1.396(6)$ \\
$\mathrm{C} 5-\mathrm{H} 5$ & $1.406(6)$ & $\mathrm{C} 30-\mathrm{C} 31$ & 0.9500 \\
$\mathrm{C} 6-\mathrm{C} 7$ & 0.9500 & & $1.508(6)$
\end{tabular}




\begin{tabular}{|c|c|c|c|}
\hline $\mathrm{C} 7-\mathrm{C} 8$ & $1.499(6)$ & $\mathrm{C} 31-\mathrm{C} 32$ & $1.503(6)$ \\
\hline $\mathrm{C} 7-\mathrm{C} 18$ & $1.499(6)$ & $\mathrm{C} 31-\mathrm{C} 42$ & $1.513(6)$ \\
\hline $\mathrm{C} 8-\mathrm{C} 13$ & $1.376(6)$ & $\mathrm{C} 32-\mathrm{C} 37$ & $1.387(6)$ \\
\hline $\mathrm{C} 8-\mathrm{C} 9$ & $1.403(6)$ & $\mathrm{C} 32-\mathrm{C} 33$ & $1.395(6)$ \\
\hline $\mathrm{C} 9-\mathrm{C} 10$ & $1.371(6)$ & $\mathrm{C} 33-\mathrm{C} 34$ & $1.370(6)$ \\
\hline C9-H9 & 0.9500 & C $33-\mathrm{H} 33$ & 0.9500 \\
\hline $\mathrm{C} 10-\mathrm{C} 11$ & $1.423(6)$ & C34-C35 & $1.418(6)$ \\
\hline $\mathrm{C} 10-\mathrm{H} 10$ & 0.9500 & C34-H34 & 0.9500 \\
\hline $\mathrm{C} 11-\mathrm{C} 12$ & $1.403(6)$ & $\mathrm{C} 35-\mathrm{C} 36$ & $1.401(6)$ \\
\hline $\mathrm{C} 12-\mathrm{C} 13$ & $1.391(6)$ & $\mathrm{C} 36-\mathrm{C} 37$ & $1.394(6)$ \\
\hline $\mathrm{C} 12-\mathrm{H} 12$ & 0.9500 & C36- & 0.9500 \\
\hline $\mathrm{C} 14-\mathrm{C} 15$ & $1.522(6)$ & C $38-\mathrm{C} 39$ & $1.525(7)$ \\
\hline $\mathrm{C} 14-\mathrm{H} 14 \mathrm{~A}$ & 0.9900 & $\mathrm{C} 38-\mathrm{H} 38 \mathrm{~A}$ & 0.9900 \\
\hline C14-H14B & 0.9900 & C $38-\mathrm{H} 38 \mathrm{~B}$ & 0.9900 \\
\hline C15-H15A & 0.9800 & C39-H39A & 0.9800 \\
\hline C15-H15B & 0.9800 & C39-H39B & 0.9800 \\
\hline $\mathrm{C} 15-\mathrm{H} 15 \mathrm{C}$ & 0.9800 & $\mathrm{C} 39-\mathrm{H} 39 \mathrm{C}$ & 0.9800 \\
\hline $\mathrm{C} 16-\mathrm{C} 17$ & $1.524(6)$ & $\mathrm{C} 40-\mathrm{C} 41$ & $1.513(6)$ \\
\hline $\mathrm{C} 16-\mathrm{H} 16 \mathrm{~A}$ & 0.9900 & $\mathrm{C} 40-\mathrm{H} 40 \mathrm{~A}$ & 0.9900 \\
\hline C16-H16B & 0.9900 & $\mathrm{C} 40-\mathrm{H} 40 \mathrm{~B}$ & 0.9900 \\
\hline C17-H17A & 0.9800 & $\mathrm{C} 41-\mathrm{H} 41 \mathrm{~A}$ & 0.9800 \\
\hline C17-H17B & 0.9800 & $\mathrm{C} 41-\mathrm{H} 41 \mathrm{~B}$ & 0.9800 \\
\hline $\mathrm{C} 17-\mathrm{H} 17 \mathrm{C}$ & 0.9800 & $\mathrm{C} 41-\mathrm{H} 41 \mathrm{C}$ & 0.9800 \\
\hline $\mathrm{C} 18-\mathrm{C} 23$ & $1.377(6)$ & $\mathrm{C} 42-\mathrm{C} 47$ & $1.382(6)$ \\
\hline $\mathrm{C} 18-\mathrm{C} 19$ & $1.398(6)$ & $\mathrm{C} 42-\mathrm{C} 43$ & $1.384(6)$ \\
\hline $\mathrm{C} 19-\mathrm{C} 20$ & $1.376(6)$ & $\mathrm{C} 43-\mathrm{C} 44$ & $1.393(6)$ \\
\hline C19-H19 & 0.9500 & $\mathrm{C} 43-\mathrm{H} 43$ & 0.9500 \\
\hline $\mathrm{C} 20-\mathrm{C} 21$ & $1.404(6)$ & $\mathrm{C} 44-\mathrm{C} 45$ & $1.388(6)$ \\
\hline $\mathrm{C} 20-\mathrm{H} 20$ & 0.9500 & $\mathrm{C} 44-\mathrm{H} 44$ & 0.9500 \\
\hline $\mathrm{C} 21-\mathrm{C} 22$ & $1.378(6)$ & $\mathrm{C} 45-\mathrm{C} 46$ & $1.386(6)$ \\
\hline $\mathrm{C} 21-\mathrm{H} 21$ & 0.9500 & $\mathrm{C} 45-\mathrm{H} 45$ & 0.9500 \\
\hline $\mathrm{C} 22-\mathrm{C} 23$ & $1.402(6)$ & $\mathrm{C} 46-\mathrm{C} 47$ & $1.386(6)$ \\
\hline $\mathrm{C} 22-\mathrm{H} 22$ & 0.9500 & $\mathrm{C} 46-\mathrm{H} 46$ & 0.9500 \\
\hline $\mathrm{C} 23-\mathrm{C} 24$ & $1.467(6)$ & $\mathrm{C} 47-\mathrm{C} 48$ & $1.478(6)$ \\
\hline $\mathrm{C} 1-\mathrm{O} 1-\mathrm{C} 13$ & $118.4(3)$ & $\mathrm{C} 25-\mathrm{O} 4-\mathrm{C} 37$ & $118.8(3)$ \\
\hline $\mathrm{C} 24-\mathrm{O} 2-\mathrm{C} 7$ & $110.6(3)$ & $\mathrm{C} 48-\mathrm{O} 5-\mathrm{C} 31$ & $111.4(3)$ \\
\hline $\mathrm{C} 11-\mathrm{N} 1-\mathrm{C} 14$ & $120.6(4)$ & $\mathrm{C} 35-\mathrm{N} 2-\mathrm{C} 38$ & $121.5(4)$ \\
\hline $\mathrm{C} 11-\mathrm{N} 1-\mathrm{C} 16$ & $120.7(4)$ & $\mathrm{C} 35-\mathrm{N} 2-\mathrm{C} 40$ & $122.7(3)$ \\
\hline $\mathrm{C} 14-\mathrm{N} 1-\mathrm{C} 16$ & $118.1(4)$ & $\mathrm{C} 38-\mathrm{N} 2-\mathrm{C} 40$ & $115.8(4)$ \\
\hline $\mathrm{O} 1-\mathrm{C} 1-\mathrm{C} 6$ & $123.5(4)$ & $\mathrm{O} 4-\mathrm{C} 25-\mathrm{C} 26$ & $115.6(4)$ \\
\hline $\mathrm{O} 1-\mathrm{C} 1-\mathrm{C} 2$ & $114.9(4)$ & $\mathrm{O} 4-\mathrm{C} 25-\mathrm{C} 30$ & $122.9(4)$ \\
\hline $\mathrm{C} 6-\mathrm{C} 1-\mathrm{C} 2$ & $121.6(4)$ & $\mathrm{C} 26-\mathrm{C} 25-\mathrm{C} 30$ & $121.5(4)$ \\
\hline $\mathrm{C} 3-\mathrm{C} 2-\mathrm{C} 1$ & $118.7(4)$ & $\mathrm{C} 27-\mathrm{C} 26-\mathrm{C} 25$ & $117.8(4)$ \\
\hline $\mathrm{C} 3-\mathrm{C} 2-\mathrm{H} 2$ & 120.7 & $\mathrm{C} 27-\mathrm{C} 26-\mathrm{H} 26$ & 121.1 \\
\hline $\mathrm{C} 1-\mathrm{C} 2-\mathrm{H} 2$ & 120.7 & $\mathrm{C} 25-\mathrm{C} 26-\mathrm{H} 26$ & 121.1 \\
\hline $\mathrm{C} 2-\mathrm{C} 3-\mathrm{C} 4$ & $121.7(4)$ & $\mathrm{C} 26-\mathrm{C} 27-\mathrm{C} 28$ & $122.8(4)$ \\
\hline $\mathrm{C} 2-\mathrm{C} 3-\mathrm{Br} 1$ & $118.9(3)$ & $\mathrm{C} 26-\mathrm{C} 27-\mathrm{Br} 2$ & $118.3(3)$ \\
\hline
\end{tabular}




\begin{tabular}{|c|c|c|c|}
\hline $\mathrm{C} 4-\mathrm{C} 3-\mathrm{Br} 1$ & $119.4(3)$ & $\mathrm{C} 28-\mathrm{C} 27-\mathrm{Br} 2$ & $118.9(3)$ \\
\hline $\mathrm{C} 5-\mathrm{C} 4-\mathrm{C} 3$ & $118.6(4)$ & $\mathrm{C} 27-\mathrm{C} 28-\mathrm{C} 29$ & $118.3(4)$ \\
\hline $\mathrm{C} 5-\mathrm{C} 4-\mathrm{H} 4$ & 120.7 & $\mathrm{C} 27-\mathrm{C} 28-\mathrm{H} 28$ & 120.9 \\
\hline $\mathrm{C} 3-\mathrm{C} 4-\mathrm{H} 4$ & 120.7 & $\mathrm{C} 29-\mathrm{C} 28-\mathrm{H} 28$ & 120.9 \\
\hline $\mathrm{C} 4-\mathrm{C} 5-\mathrm{C} 6$ & $121.1(4)$ & $\mathrm{C} 28-\mathrm{C} 29-\mathrm{C} 30$ & $121.2(4)$ \\
\hline $\mathrm{C} 4-\mathrm{C} 5-\mathrm{H} 5$ & 119.4 & $\mathrm{C} 28-\mathrm{C} 29-\mathrm{H} 29$ & 119.4 \\
\hline $\mathrm{C} 6-\mathrm{C} 5-\mathrm{H} 5$ & 119.4 & $\mathrm{C} 30-\mathrm{C} 29-\mathrm{H} 29$ & 119.4 \\
\hline $\mathrm{C} 1-\mathrm{C} 6-\mathrm{C} 5$ & $118.2(4)$ & $\mathrm{C} 25-\mathrm{C} 30-\mathrm{C} 29$ & $118.4(4)$ \\
\hline $\mathrm{C} 1-\mathrm{C} 6-\mathrm{C} 7$ & $121.3(4)$ & $\mathrm{C} 25-\mathrm{C} 30-\mathrm{C} 31$ & $121.8(4)$ \\
\hline $\mathrm{C} 5-\mathrm{C} 6-\mathrm{C} 7$ & $120.2(4)$ & $\mathrm{C} 29-\mathrm{C} 30-\mathrm{C} 31$ & $119.8(4)$ \\
\hline $\mathrm{C} 8-\mathrm{C} 7-\mathrm{C} 18$ & $113.9(4)$ & $\mathrm{O} 5-\mathrm{C} 31-\mathrm{C} 32$ & $108.6(3)$ \\
\hline $\mathrm{C} 8-\mathrm{C} 7-\mathrm{O} 2$ & $109.8(3)$ & $\mathrm{O} 5-\mathrm{C} 31-\mathrm{C} 30$ & $108.8(3)$ \\
\hline $\mathrm{C} 18-\mathrm{C} 7-\mathrm{O} 2$ & $101.8(3)$ & $\mathrm{C} 32-\mathrm{C} 31-\mathrm{C} 30$ & $111.1(3)$ \\
\hline $\mathrm{C} 8-\mathrm{C} 7-\mathrm{C} 6$ & $110.6(3)$ & $\mathrm{O} 5-\mathrm{C} 31-\mathrm{C} 42$ & $102.2(3)$ \\
\hline $\mathrm{C} 18-\mathrm{C} 7-\mathrm{C} 6$ & $115.5(3)$ & $\mathrm{C} 32-\mathrm{C} 31-\mathrm{C} 42$ & $113.0(3)$ \\
\hline $\mathrm{O} 2-\mathrm{C} 7-\mathrm{C} 6$ & $104.3(3)$ & $\mathrm{C} 30-\mathrm{C} 31-\mathrm{C} 42$ & $112.6(3)$ \\
\hline $\mathrm{C} 13-\mathrm{C} 8-\mathrm{C} 9$ & $115.8(4)$ & $\mathrm{C} 37-\mathrm{C} 32-\mathrm{C} 33$ & $116.3(4)$ \\
\hline $\mathrm{C} 13-\mathrm{C} 8-\mathrm{C} 7$ & $122.7(4)$ & $\mathrm{C} 37-\mathrm{C} 32-\mathrm{C} 31$ & $122.6(4)$ \\
\hline $\mathrm{C} 9-\mathrm{C} 8-\mathrm{C} 7$ & $121.4(4)$ & $\mathrm{C} 33-\mathrm{C} 32-\mathrm{C} 31$ & $121.0(4)$ \\
\hline $\mathrm{C} 10-\mathrm{C} 9-\mathrm{C} 8$ & $122.7(4)$ & $\mathrm{C} 34-\mathrm{C} 33-\mathrm{C} 32$ & $122.2(4)$ \\
\hline $\mathrm{C} 10-\mathrm{C} 9-\mathrm{H} 9$ & 118.7 & $\mathrm{C} 34-\mathrm{C} 33-\mathrm{H} 33$ & 118.9 \\
\hline $\mathrm{C} 8-\mathrm{C} 9-\mathrm{H} 9$ & 118.7 & $\mathrm{C} 32-\mathrm{C} 33-\mathrm{H} 33$ & 118.9 \\
\hline $\mathrm{C} 9-\mathrm{C} 10-\mathrm{C} 11$ & $120.7(4)$ & $\mathrm{C} 33-\mathrm{C} 34-\mathrm{C} 35$ & $121.5(4)$ \\
\hline $\mathrm{C} 9-\mathrm{C} 10-\mathrm{H} 10$ & 119.6 & $\mathrm{C} 33-\mathrm{C} 34-\mathrm{H} 34$ & 119.2 \\
\hline $\mathrm{C} 11-\mathrm{C} 10-\mathrm{H} 10$ & 119.6 & $\mathrm{C} 35-\mathrm{C} 34-\mathrm{H} 34$ & 119.2 \\
\hline $\mathrm{N} 1-\mathrm{C} 11-\mathrm{C} 12$ & $121.1(4)$ & $\mathrm{N} 2-\mathrm{C} 35-\mathrm{C} 36$ & $121.7(4)$ \\
\hline $\mathrm{N} 1-\mathrm{C} 11-\mathrm{C} 10$ & $121.7(4)$ & $\mathrm{N} 2-\mathrm{C} 35-\mathrm{C} 34$ & $121.6(4)$ \\
\hline $\mathrm{C} 12-\mathrm{C} 11-\mathrm{C} 10$ & $117.1(4)$ & $\mathrm{C} 36-\mathrm{C} 35-\mathrm{C} 34$ & $116.6(4)$ \\
\hline $\mathrm{C} 13-\mathrm{C} 12-\mathrm{C} 11$ & $119.8(4)$ & $\mathrm{C} 37-\mathrm{C} 36-\mathrm{C} 35$ & $120.4(4)$ \\
\hline $\mathrm{C} 13-\mathrm{C} 12-\mathrm{H} 12$ & 120.1 & $\mathrm{C} 37-\mathrm{C} 36-\mathrm{H} 36$ & 119.8 \\
\hline $\mathrm{C} 11-\mathrm{C} 12-\mathrm{H} 12$ & 120.1 & $\mathrm{C} 35-\mathrm{C} 36-\mathrm{H} 36$ & 119.8 \\
\hline $\mathrm{C} 8-\mathrm{C} 13-\mathrm{O} 1$ & $122.2(4)$ & $\mathrm{O} 4-\mathrm{C} 37-\mathrm{C} 32$ & $122.5(4)$ \\
\hline $\mathrm{C} 8-\mathrm{C} 13-\mathrm{C} 12$ & $123.8(4)$ & $\mathrm{O} 4-\mathrm{C} 37-\mathrm{C} 36$ & $114.7(4)$ \\
\hline $\mathrm{O} 1-\mathrm{C} 13-\mathrm{C} 12$ & $114.0(4)$ & $\mathrm{C} 32-\mathrm{C} 37-\mathrm{C} 36$ & $122.8(4)$ \\
\hline $\mathrm{N} 1-\mathrm{C} 14-\mathrm{C} 15$ & $112.9(4)$ & $\mathrm{N} 2-\mathrm{C} 38-\mathrm{C} 39$ & $113.8(4)$ \\
\hline $\mathrm{N} 1-\mathrm{C} 14-\mathrm{H} 14 \mathrm{~A}$ & 109.0 & $\mathrm{~N} 2-\mathrm{C} 38-\mathrm{H} 38 \mathrm{~A}$ & 108.8 \\
\hline $\mathrm{C} 15-\mathrm{C} 14-\mathrm{H} 14 \mathrm{~A}$ & 109.0 & $\mathrm{C} 39-\mathrm{C} 38-\mathrm{H} 38 \mathrm{~A}$ & 108.8 \\
\hline $\mathrm{N} 1-\mathrm{C} 14-\mathrm{H} 14 \mathrm{~B}$ & 109.0 & $\mathrm{~N} 2-\mathrm{C} 38-\mathrm{H} 38 \mathrm{~B}$ & 108.8 \\
\hline $\mathrm{C} 15-\mathrm{C} 14-\mathrm{H} 14 \mathrm{~B}$ & 109.0 & $\mathrm{C} 39-\mathrm{C} 38-\mathrm{H} 38 \mathrm{~B}$ & 108.8 \\
\hline $\mathrm{H} 14 \mathrm{~A}-\mathrm{C} 14-\mathrm{H} 14 \mathrm{~B}$ & 107.8 & $\mathrm{H} 38 \mathrm{~A}-\mathrm{C} 38-\mathrm{H} 38 \mathrm{~B}$ & 107.7 \\
\hline $\mathrm{C} 14-\mathrm{C} 15-\mathrm{H} 15 \mathrm{~A}$ & 109.5 & $\mathrm{C} 38-\mathrm{C} 39-\mathrm{H} 39 \mathrm{~A}$ & 109.5 \\
\hline $\mathrm{C} 14-\mathrm{C} 15-\mathrm{H} 15 \mathrm{~B}$ & 109.5 & $\mathrm{C} 38-\mathrm{C} 39-\mathrm{H} 39 \mathrm{~B}$ & 109.5 \\
\hline $\mathrm{H} 15 \mathrm{~A}-\mathrm{C} 15-\mathrm{H} 15 \mathrm{~B}$ & 109.5 & $\mathrm{H} 39 \mathrm{~A}-\mathrm{C} 39-\mathrm{H} 39 \mathrm{~B}$ & 109.5 \\
\hline $\mathrm{C} 14-\mathrm{C} 15-\mathrm{H} 15 \mathrm{C}$ & 109.5 & $\mathrm{C} 38-\mathrm{C} 39-\mathrm{H} 39 \mathrm{C}$ & 109.5 \\
\hline $\mathrm{H} 15 \mathrm{~A}-\mathrm{C} 15-\mathrm{H} 15 \mathrm{C}$ & 109.5 & $\mathrm{H} 39 \mathrm{~A}-\mathrm{C} 39-\mathrm{H} 39 \mathrm{C}$ & 109.5 \\
\hline $\mathrm{H} 15 \mathrm{~B}-\mathrm{C} 15-\mathrm{H} 15 \mathrm{C}$ & 109.5 & $\mathrm{H} 39 \mathrm{~B}-\mathrm{C} 39-\mathrm{H} 39 \mathrm{C}$ & 109.5 \\
\hline $\mathrm{N} 1-\mathrm{C} 16-\mathrm{C} 17$ & $114.1(4)$ & $\mathrm{N} 2-\mathrm{C} 40-\mathrm{C} 41$ & $114.3(4)$ \\
\hline $\mathrm{N} 1-\mathrm{C} 16-\mathrm{H} 16 \mathrm{~A}$ & 108.7 & $\mathrm{~N} 2-\mathrm{C} 40-\mathrm{H} 40 \mathrm{~A}$ & 108.7 \\
\hline
\end{tabular}




\begin{tabular}{|c|c|c|c|}
\hline $\mathrm{C} 17-\mathrm{C} 16-\mathrm{H} 16 \mathrm{~A}$ & 108.7 & $\mathrm{C} 41-\mathrm{C} 40-\mathrm{H} 40 \mathrm{~A}$ & 108.7 \\
\hline $\mathrm{N} 1-\mathrm{C} 16-\mathrm{H} 16 \mathrm{~B}$ & 108.7 & $\mathrm{~N} 2-\mathrm{C} 40-\mathrm{H} 40 \mathrm{~B}$ & 108.7 \\
\hline $\mathrm{C} 17-\mathrm{C} 16-\mathrm{H} 16 \mathrm{~B}$ & 108.7 & $\mathrm{C} 41-\mathrm{C} 40-\mathrm{H} 40 \mathrm{~B}$ & 108.7 \\
\hline $\mathrm{H} 16 \mathrm{~A}-\mathrm{C} 16-\mathrm{H} 16 \mathrm{~B}$ & 107.6 & $\mathrm{H} 40 \mathrm{~A}-\mathrm{C} 40-\mathrm{H} 40 \mathrm{~B}$ & 107.6 \\
\hline $\mathrm{C} 16-\mathrm{C} 17-\mathrm{H} 17 \mathrm{~A}$ & 109.5 & $\mathrm{C} 40-\mathrm{C} 41-\mathrm{H} 41 \mathrm{~A}$ & 109.5 \\
\hline $\mathrm{C} 16-\mathrm{C} 17-\mathrm{H} 17 \mathrm{~B}$ & 109.5 & $\mathrm{C} 40-\mathrm{C} 41-\mathrm{H} 41 \mathrm{~B}$ & 109.5 \\
\hline $\mathrm{H} 17 \mathrm{~A}-\mathrm{C} 17-\mathrm{H} 17 \mathrm{~B}$ & 109.5 & $\mathrm{H} 41 \mathrm{~A}-\mathrm{C} 41-\mathrm{H} 41 \mathrm{~B}$ & 109.5 \\
\hline $\mathrm{C} 16-\mathrm{C} 17-\mathrm{H} 17 \mathrm{C}$ & 109.5 & $\mathrm{C} 40-\mathrm{C} 41-\mathrm{H} 41 \mathrm{C}$ & 109.5 \\
\hline $\mathrm{H} 17 \mathrm{~A}-\mathrm{C} 17-\mathrm{H} 17 \mathrm{C}$ & 109.5 & $\mathrm{H} 41 \mathrm{~A}-\mathrm{C} 41-\mathrm{H} 41 \mathrm{C}$ & 109.5 \\
\hline $\mathrm{H} 17 \mathrm{~B}-\mathrm{C} 17-\mathrm{H} 17 \mathrm{C}$ & 109.5 & $\mathrm{H} 41 \mathrm{~B}-\mathrm{C} 41-\mathrm{H} 41 \mathrm{C}$ & 109.5 \\
\hline $\mathrm{C} 23-\mathrm{C} 18-\mathrm{C} 19$ & $120.7(4)$ & $\mathrm{C} 47-\mathrm{C} 42-\mathrm{C} 43$ & $120.6(4)$ \\
\hline $\mathrm{C} 23-\mathrm{C} 18-\mathrm{C} 7$ & $110.5(4)$ & $\mathrm{C} 47-\mathrm{C} 42-\mathrm{C} 31$ & $110.0(4)$ \\
\hline $\mathrm{C} 19-\mathrm{C} 18-\mathrm{C} 7$ & $128.8(4)$ & $\mathrm{C} 43-\mathrm{C} 42-\mathrm{C} 31$ & $129.4(4)$ \\
\hline $\mathrm{C} 20-\mathrm{C} 19-\mathrm{C} 18$ & $117.5(4)$ & $\mathrm{C} 42-\mathrm{C} 43-\mathrm{C} 44$ & $117.8(4)$ \\
\hline $\mathrm{C} 20-\mathrm{C} 19-\mathrm{H} 19$ & 121.2 & $\mathrm{C} 42-\mathrm{C} 43-\mathrm{H} 43$ & 121.1 \\
\hline $\mathrm{C} 18-\mathrm{C} 19-\mathrm{H} 19$ & 121.2 & $\mathrm{C} 44-\mathrm{C} 43-\mathrm{H} 43$ & 121.1 \\
\hline $\mathrm{C} 19-\mathrm{C} 20-\mathrm{C} 21$ & $121.7(4)$ & $\mathrm{C} 45-\mathrm{C} 44-\mathrm{C} 43$ & $121.3(4)$ \\
\hline $\mathrm{C} 19-\mathrm{C} 20-\mathrm{H} 20$ & 119.2 & $\mathrm{C} 45-\mathrm{C} 44-\mathrm{H} 44$ & 119.4 \\
\hline $\mathrm{C} 21-\mathrm{C} 20-\mathrm{H} 20$ & 119.2 & $\mathrm{C} 43-\mathrm{C} 44-\mathrm{H} 44$ & 119.4 \\
\hline $\mathrm{C} 22-\mathrm{C} 21-\mathrm{C} 20$ & $121.0(4)$ & $\mathrm{C} 46-\mathrm{C} 45-\mathrm{C} 44$ & $120.7(4)$ \\
\hline $\mathrm{C} 22-\mathrm{C} 21-\mathrm{H} 21$ & 119.5 & $\mathrm{C} 46-\mathrm{C} 45-\mathrm{H} 45$ & 119.7 \\
\hline $\mathrm{C} 20-\mathrm{C} 21-\mathrm{H} 21$ & 119.5 & $\mathrm{C} 44-\mathrm{C} 45-\mathrm{H} 45$ & 119.7 \\
\hline $\mathrm{C} 21-\mathrm{C} 22-\mathrm{C} 23$ & $116.9(4)$ & $\mathrm{C} 47-\mathrm{C} 46-\mathrm{C} 45$ & $117.6(4)$ \\
\hline $\mathrm{C} 21-\mathrm{C} 22-\mathrm{H} 22$ & 121.5 & $\mathrm{C} 47-\mathrm{C} 46-\mathrm{H} 46$ & 121.2 \\
\hline $\mathrm{C} 23-\mathrm{C} 22-\mathrm{H} 22$ & 121.5 & $\mathrm{C} 45-\mathrm{C} 46-\mathrm{H} 46$ & 121.2 \\
\hline $\mathrm{C} 18-\mathrm{C} 23-\mathrm{C} 22$ & $122.1(4)$ & $\mathrm{C} 42-\mathrm{C} 47-\mathrm{C} 46$ & $121.9(4)$ \\
\hline $\mathrm{C} 18-\mathrm{C} 23-\mathrm{C} 24$ & $108.9(4)$ & $\mathrm{C} 42-\mathrm{C} 47-\mathrm{C} 48$ & $108.2(4)$ \\
\hline $\mathrm{C} 22-\mathrm{C} 23-\mathrm{C} 24$ & $129.0(4)$ & $\mathrm{C} 46-\mathrm{C} 47-\mathrm{C} 48$ & $129.9(4)$ \\
\hline $\mathrm{O} 3-\mathrm{C} 24-\mathrm{O} 2$ & $121.1(4)$ & $\mathrm{O} 6-\mathrm{C} 48-\mathrm{O} 5$ & $121.3(4)$ \\
\hline $\mathrm{O} 3-\mathrm{C} 24-\mathrm{C} 23$ & $131.2(4)$ & $\mathrm{O} 6-\mathrm{C} 48-\mathrm{C} 47$ & $130.7(4)$ \\
\hline $\mathrm{O} 2-\mathrm{C} 24-\mathrm{C} 23$ & $107.7(4)$ & $\mathrm{O} 5-\mathrm{C} 48-\mathrm{C} 47$ & $108.0(4)$ \\
\hline $\mathrm{C} 13-\mathrm{O} 1-\mathrm{C} 1-\mathrm{C} 6$ & $3.3(6)$ & $\mathrm{C} 37-\mathrm{O} 4-\mathrm{C} 25-\mathrm{C} 26$ & $-177.1(4)$ \\
\hline $\mathrm{C} 13-\mathrm{O} 1-\mathrm{C} 1-\mathrm{C} 2$ & $-177.9(4)$ & $\mathrm{C} 37-\mathrm{O} 4-\mathrm{C} 25-\mathrm{C} 30$ & $4.2(6)$ \\
\hline $\mathrm{O} 1-\mathrm{C} 1-\mathrm{C} 2-\mathrm{C} 3$ & $-179.0(4)$ & $\mathrm{O} 4-\mathrm{C} 25-\mathrm{C} 26-\mathrm{C} 27$ & $-177.7(4)$ \\
\hline $\mathrm{C} 6-\mathrm{C} 1-\mathrm{C} 2-\mathrm{C} 3$ & $-0.2(7)$ & $\mathrm{C} 30-\mathrm{C} 25-\mathrm{C} 26-\mathrm{C} 27$ & $1.1(6)$ \\
\hline $\mathrm{C} 1-\mathrm{C} 2-\mathrm{C} 3-\mathrm{C} 4$ & $-2.1(7)$ & $\mathrm{C} 25-\mathrm{C} 26-\mathrm{C} 27-\mathrm{C} 28$ & $1.1(7)$ \\
\hline $\mathrm{C} 1-\mathrm{C} 2-\mathrm{C} 3-\mathrm{Br} 1$ & $177.4(3)$ & $\mathrm{C} 25-\mathrm{C} 26-\mathrm{C} 27-\mathrm{Br} 2$ & $-178.9(3)$ \\
\hline $\mathrm{C} 2-\mathrm{C} 3-\mathrm{C} 4-\mathrm{C} 5$ & $1.8(7)$ & $\mathrm{C} 26-\mathrm{C} 27-\mathrm{C} 28-\mathrm{C} 29$ & $-2.1(7)$ \\
\hline $\mathrm{Br} 1-\mathrm{C} 3-\mathrm{C} 4-\mathrm{C} 5$ & $-177.7(3)$ & $\mathrm{Br} 2-\mathrm{C} 27-\mathrm{C} 28-\mathrm{C} 29$ & $177.9(3)$ \\
\hline $\mathrm{C} 3-\mathrm{C} 4-\mathrm{C} 5-\mathrm{C} 6$ & $0.8(7)$ & $\mathrm{C} 27-\mathrm{C} 28-\mathrm{C} 29-\mathrm{C} 30$ & $1.0(7)$ \\
\hline $\mathrm{O} 1-\mathrm{C} 1-\mathrm{C} 6-\mathrm{C} 5$ & $-178.7(4)$ & $\mathrm{O} 4-\mathrm{C} 25-\mathrm{C} 30-\mathrm{C} 29$ & $176.6(4)$ \\
\hline $\mathrm{C} 2-\mathrm{C} 1-\mathrm{C} 6-\mathrm{C} 5$ & $2.7(7)$ & $\mathrm{C} 26-\mathrm{C} 25-\mathrm{C} 30-\mathrm{C} 29$ & $-2.1(6)$ \\
\hline $\mathrm{O} 1-\mathrm{C} 1-\mathrm{C} 6-\mathrm{C} 7$ & $7.1(7)$ & $\mathrm{O} 4-\mathrm{C} 25-\mathrm{C} 30-\mathrm{C} 31$ & $-4.8(6)$ \\
\hline $\mathrm{C} 2-\mathrm{C} 1-\mathrm{C} 6-\mathrm{C} 7$ & $-171.5(4)$ & $\mathrm{C} 26-\mathrm{C} 25-\mathrm{C} 30-\mathrm{C} 31$ & $176.5(4)$ \\
\hline $\mathrm{C} 4-\mathrm{C} 5-\mathrm{C} 6-\mathrm{C} 1$ & $-3.0(7)$ & $\mathrm{C} 28-\mathrm{C} 29-\mathrm{C} 30-\mathrm{C} 25$ & $1.0(7)$ \\
\hline $\mathrm{C} 4-\mathrm{C} 5-\mathrm{C} 6-\mathrm{C} 7$ & $171.3(4)$ & $\mathrm{C} 28-\mathrm{C} 29-\mathrm{C} 30-\mathrm{C} 31$ & $-177.6(4)$ \\
\hline $\mathrm{C} 24-\mathrm{O} 2-\mathrm{C} 7-\mathrm{C} 8$ & $-128.9(4)$ & $\mathrm{C} 48-\mathrm{O} 5-\mathrm{C} 31-\mathrm{C} 32$ & $-116.1(4)$ \\
\hline
\end{tabular}




\begin{tabular}{|c|c|}
\hline $\mathrm{C} 24-\mathrm{O} 2-\mathrm{C} 7-\mathrm{C} 18$ & $-7.9(4)$ \\
\hline $\mathrm{C} 24-\mathrm{O} 2-\mathrm{C} 7-\mathrm{C} 6$ & $112.6(3)$ \\
\hline $\mathrm{C} 1-\mathrm{C} 6-\mathrm{C} 7-\mathrm{C} 8$ & $-12.5(6)$ \\
\hline $\mathrm{C} 5-\mathrm{C} 6-\mathrm{C} 7-\mathrm{C} 8$ & $173.4(4)$ \\
\hline $\mathrm{C} 1-\mathrm{C} 6-\mathrm{C} 7-\mathrm{C} 18$ & $-143.7(4)$ \\
\hline $\mathrm{C} 5-\mathrm{C} 6-\mathrm{C} 7-\mathrm{C} 18$ & $42.2(5)$ \\
\hline $\mathrm{C} 1-\mathrm{C} 6-\mathrm{C} 7-\mathrm{O} 2$ & $105.5(4)$ \\
\hline $\mathrm{C} 5-\mathrm{C} 6-\mathrm{C} 7-\mathrm{O} 2$ & $-68.6(5)$ \\
\hline $\mathrm{C} 18-\mathrm{C} 7-\mathrm{C} 8-\mathrm{C} 13$ & $141.0(4)$ \\
\hline $\mathrm{O} 2-\mathrm{C} 7-\mathrm{C} 8-\mathrm{C} 13$ & $-105.6(4)$ \\
\hline $\mathrm{C} 6-\mathrm{C} 7-\mathrm{C} 8-\mathrm{C} 13$ & $9.0(6)$ \\
\hline $\mathrm{C} 18-\mathrm{C} 7-\mathrm{C} 8-\mathrm{C} 9$ & $-39.6(5)$ \\
\hline $\mathrm{O} 2-\mathrm{C} 7-\mathrm{C} 8-\mathrm{C} 9$ & $73.7(5)$ \\
\hline $\mathrm{C} 6-\mathrm{C} 7-\mathrm{C} 8-\mathrm{C} 9$ & $-171.7(4)$ \\
\hline $\mathrm{C} 13-\mathrm{C} 8-\mathrm{C} 9-\mathrm{C} 10$ & $-0.3(6)$ \\
\hline $\mathrm{C} 7-\mathrm{C} 8-\mathrm{C} 9-\mathrm{C} 10$ & $-179.7(4)$ \\
\hline $\mathrm{C} 8-\mathrm{C} 9-\mathrm{C} 10-\mathrm{C} 11$ & $-1.4(7)$ \\
\hline $\mathrm{C} 14-\mathrm{N} 1-\mathrm{C} 11-\mathrm{C} 12$ & $174.3(4)$ \\
\hline $\mathrm{C} 16-\mathrm{N} 1-\mathrm{C} 11-\mathrm{C} 12$ & $2.9(6)$ \\
\hline $\mathrm{C} 14-\mathrm{N} 1-\mathrm{C} 11-\mathrm{C} 10$ & $-9.5(6)$ \\
\hline $\mathrm{C} 16-\mathrm{N} 1-\mathrm{C} 11-\mathrm{C} 10$ & $179.0(4)$ \\
\hline $\mathrm{C} 9-\mathrm{C} 10-\mathrm{C} 11-\mathrm{N} 1$ & $-173.3(4)$ \\
\hline $\mathrm{C} 9-\mathrm{C} 10-\mathrm{C} 11-\mathrm{C} 12$ & $3.0(6)$ \\
\hline $\mathrm{N} 1-\mathrm{C} 11-\mathrm{C} 12-\mathrm{C} 13$ & $173.2(4)$ \\
\hline $\mathrm{C} 10-\mathrm{C} 11-\mathrm{C} 12-\mathrm{C} 13$ & $-3.1(6)$ \\
\hline $\mathrm{C} 9-\mathrm{C} 8-\mathrm{C} 13-\mathrm{O} 1$ & $-179.1(4)$ \\
\hline $\mathrm{C} 7-\mathrm{C} 8-\mathrm{C} 13-\mathrm{O} 1$ & $0.3(6)$ \\
\hline $\mathrm{C} 9-\mathrm{C} 8-\mathrm{C} 13-\mathrm{C} 12$ & $0.2(6)$ \\
\hline $\mathrm{C} 7-\mathrm{C} 8-\mathrm{C} 13-\mathrm{C} 12$ & $179.6(4)$ \\
\hline $\mathrm{C} 1-\mathrm{O} 1-\mathrm{C} 13-\mathrm{C} 8$ & $-7.1(6)$ \\
\hline $\mathrm{C} 1-\mathrm{O} 1-\mathrm{C} 13-\mathrm{C} 12$ & $173.5(4)$ \\
\hline $\mathrm{C} 11-\mathrm{C} 12-\mathrm{C} 13-\mathrm{C} 8$ & $1.6(7)$ \\
\hline $\mathrm{C} 11-\mathrm{C} 12-\mathrm{C} 13-\mathrm{O} 1$ & $-179.1(4)$ \\
\hline $\mathrm{C} 11-\mathrm{N} 1-\mathrm{C} 14-\mathrm{C} 15$ & $-77.4(5)$ \\
\hline $\mathrm{C} 16-\mathrm{N} 1-\mathrm{C} 14-\mathrm{C} 15$ & $94.2(5)$ \\
\hline $\mathrm{C} 11-\mathrm{N} 1-\mathrm{C} 16-\mathrm{C} 17$ & $76.2(5)$ \\
\hline $\mathrm{C} 14-\mathrm{N} 1-\mathrm{C} 16-\mathrm{C} 17$ & $-95.4(5)$ \\
\hline $\mathrm{C} 8-\mathrm{C} 7-\mathrm{C} 18-\mathrm{C} 23$ & $122.5(4)$ \\
\hline $\mathrm{O} 2-\mathrm{C} 7-\mathrm{C} 18-\mathrm{C} 23$ & $4.4(4)$ \\
\hline $\mathrm{C} 6-\mathrm{C} 7-\mathrm{C} 18-\mathrm{C} 23$ & $-107.9(4)$ \\
\hline $\mathrm{C} 8-\mathrm{C} 7-\mathrm{C} 18-\mathrm{C} 19$ & $-54.7(6)$ \\
\hline $\mathrm{O} 2-\mathrm{C} 7-\mathrm{C} 18-\mathrm{C} 19$ & $-172.8(4)$ \\
\hline $\mathrm{C} 6-\mathrm{C} 7-\mathrm{C} 18-\mathrm{C} 19$ & $74.9(6)$ \\
\hline $\mathrm{C} 23-\mathrm{C} 18-\mathrm{C} 19-\mathrm{C} 20$ & $0.8(6)$ \\
\hline $\mathrm{C} 7-\mathrm{C} 18-\mathrm{C} 19-\mathrm{C} 20$ & $177.7(4)$ \\
\hline $\mathrm{C} 18-\mathrm{C} 19-\mathrm{C} 20-\mathrm{C} 21$ & $-0.7(6)$ \\
\hline $\mathrm{C} 19-\mathrm{C} 20-\mathrm{C} 21-\mathrm{C} 22$ & $0.4(7)$ \\
\hline $\mathrm{C} 20-\mathrm{C} 21-\mathrm{C} 22-\mathrm{C} 23$ & $-0.2(6)$ \\
\hline
\end{tabular}

\begin{tabular}{|c|c|}
\hline $\mathrm{C} 48-\mathrm{O} 5-\mathrm{C} 31-\mathrm{C} 30$ & $122.8(3)$ \\
\hline $\mathrm{C} 48-\mathrm{O} 5-\mathrm{C} 31-\mathrm{C} 42$ & $3.5(4)$ \\
\hline $\mathrm{C} 25-\mathrm{C} 30-\mathrm{C} 31-\mathrm{O} 5$ & $122.8(4)$ \\
\hline $\mathrm{C} 29-\mathrm{C} 30-\mathrm{C} 31-\mathrm{O} 5$ & $-58.7(5)$ \\
\hline $\mathrm{C} 25-\mathrm{C} 30-\mathrm{C} 31-\mathrm{C} 32$ & $3.3(5)$ \\
\hline $\mathrm{C} 29-\mathrm{C} 30-\mathrm{C} 31-\mathrm{C} 32$ & $-178.2(4)$ \\
\hline $\mathrm{C} 25-\mathrm{C} 30-\mathrm{C} 31-\mathrm{C} 42$ & $-124.7(4)$ \\
\hline $\mathrm{C} 29-\mathrm{C} 30-\mathrm{C} 31-\mathrm{C} 42$ & $53.8(5)$ \\
\hline $\mathrm{O} 5-\mathrm{C} 31-\mathrm{C} 32-\mathrm{C} 37$ & $-121.2(4)$ \\
\hline $\mathrm{C} 30-\mathrm{C} 31-\mathrm{C} 32-\mathrm{C} 37$ & $-1.6(5)$ \\
\hline $\mathrm{C} 42-\mathrm{C} 31-\mathrm{C} 32-\mathrm{C} 37$ & $126.2(4)$ \\
\hline $\mathrm{O} 5-\mathrm{C} 31-\mathrm{C} 32-\mathrm{C} 33$ & $60.6(5)$ \\
\hline $\mathrm{C} 30-\mathrm{C} 31-\mathrm{C} 32-\mathrm{C} 33$ & $-179.8(4)$ \\
\hline $\mathrm{C} 42-\mathrm{C} 31-\mathrm{C} 32-\mathrm{C} 33$ & $-52.0(5)$ \\
\hline $\mathrm{C} 37-\mathrm{C} 32-\mathrm{C} 33-\mathrm{C} 34$ & $0.9(6)$ \\
\hline $\mathrm{C} 31-\mathrm{C} 32-\mathrm{C} 33-\mathrm{C} 34$ & $179.1(4)$ \\
\hline $\mathrm{C} 32-\mathrm{C} 33-\mathrm{C} 34-\mathrm{C} 35$ & $-1.1(7)$ \\
\hline $\mathrm{C} 38-\mathrm{N} 2-\mathrm{C} 35-\mathrm{C} 36$ & $168.1(4)$ \\
\hline $\mathrm{C} 40-\mathrm{N} 2-\mathrm{C} 35-\mathrm{C} 36$ & $-10.5(6)$ \\
\hline $\mathrm{C} 38-\mathrm{N} 2-\mathrm{C} 35-\mathrm{C} 34$ & $-11.8(6)$ \\
\hline $\mathrm{C} 40-\mathrm{N} 2-\mathrm{C} 35-\mathrm{C} 34$ & $169.5(4)$ \\
\hline $\mathrm{C} 33-\mathrm{C} 34-\mathrm{C} 35-\mathrm{N} 2$ & $-179.9(4)$ \\
\hline $\mathrm{C} 33-\mathrm{C} 34-\mathrm{C} 35-\mathrm{C} 36$ & $0.1(6)$ \\
\hline $\mathrm{N} 2-\mathrm{C} 35-\mathrm{C} 36-\mathrm{C} 37$ & $-178.9(4)$ \\
\hline $\mathrm{C} 34-\mathrm{C} 35-\mathrm{C} 36-\mathrm{C} 37$ & $1.1(6)$ \\
\hline $\mathrm{C} 25-\mathrm{O} 4-\mathrm{C} 37-\mathrm{C} 32$ & $-2.4(6)$ \\
\hline $\mathrm{C} 25-\mathrm{O} 4-\mathrm{C} 37-\mathrm{C} 36$ & $176.8(4)$ \\
\hline $\mathrm{C} 33-\mathrm{C} 32-\mathrm{C} 37-\mathrm{O} 4$ & $179.5(4)$ \\
\hline $\mathrm{C} 31-\mathrm{C} 32-\mathrm{C} 37-\mathrm{O} 4$ & $1.3(6)$ \\
\hline $\mathrm{C} 33-\mathrm{C} 32-\mathrm{C} 37-\mathrm{C} 36$ & $0.4(6)$ \\
\hline $\mathrm{C} 31-\mathrm{C} 32-\mathrm{C} 37-\mathrm{C} 36$ & $-177.9(4)$ \\
\hline $\mathrm{C} 35-\mathrm{C} 36-\mathrm{C} 37-\mathrm{O} 4$ & $179.4(4)$ \\
\hline $\mathrm{C} 35-\mathrm{C} 36-\mathrm{C} 37-\mathrm{C} 32$ & $-1.4(7)$ \\
\hline $\mathrm{C} 35-\mathrm{N} 2-\mathrm{C} 38-\mathrm{C} 39$ & $91.9(5)$ \\
\hline $\mathrm{C} 40-\mathrm{N} 2-\mathrm{C} 38-\mathrm{C} 39$ & $-89.4(5)$ \\
\hline $\mathrm{C} 35-\mathrm{N} 2-\mathrm{C} 40-\mathrm{C} 41$ & $90.8(5)$ \\
\hline $\mathrm{C} 38-\mathrm{N} 2-\mathrm{C} 40-\mathrm{C} 41$ & $-87.9(5)$ \\
\hline $\mathrm{O} 5-\mathrm{C} 31-\mathrm{C} 42-\mathrm{C} 47$ & $-5.1(4)$ \\
\hline $\mathrm{C} 32-\mathrm{C} 31-\mathrm{C} 42-\mathrm{C} 47$ & $111.4(4)$ \\
\hline $\mathrm{C} 30-\mathrm{C} 31-\mathrm{C} 42-\mathrm{C} 47$ & $-121.7(4)$ \\
\hline $\mathrm{O} 5-\mathrm{C} 31-\mathrm{C} 42-\mathrm{C} 43$ & $175.9(4)$ \\
\hline $\mathrm{C} 32-\mathrm{C} 31-\mathrm{C} 42-\mathrm{C} 43$ & $-67.6(6)$ \\
\hline $\mathrm{C} 30-\mathrm{C} 31-\mathrm{C} 42-\mathrm{C} 43$ & $59.3(6)$ \\
\hline $\mathrm{C} 47-\mathrm{C} 42-\mathrm{C} 43-\mathrm{C} 44$ & $-1.4(7)$ \\
\hline $\mathrm{C} 31-\mathrm{C} 42-\mathrm{C} 43-\mathrm{C} 44$ & $177.5(4)$ \\
\hline $\mathrm{C} 42-\mathrm{C} 43-\mathrm{C} 44-\mathrm{C} 45$ & $-1.4(7)$ \\
\hline $\mathrm{C} 43-\mathrm{C} 44-\mathrm{C} 45-\mathrm{C} 46$ & $3.4(7)$ \\
\hline $\mathrm{C} 44-\mathrm{C} 45-\mathrm{C} 46-\mathrm{C} 47$ & $-2.4(6)$ \\
\hline
\end{tabular}




$\begin{array}{ll}\mathrm{C} 19-\mathrm{C} 18-\mathrm{C} 23-\mathrm{C} 22 & -0.7(6) \\ \mathrm{C} 7-\mathrm{C} 18-\mathrm{C} 23-\mathrm{C} 22 & -178.1(4) \\ \mathrm{C} 19-\mathrm{C} 18-\mathrm{C} 23-\mathrm{C} 24 & 177.7(4) \\ \mathrm{C} 7-\mathrm{C} 18-\mathrm{C} 23-\mathrm{C} 24 & 0.3(5) \\ \mathrm{C} 21-\mathrm{C} 22-\mathrm{C} 23-\mathrm{C} 18 & 0.4(6) \\ \mathrm{C} 21-\mathrm{C} 22-\mathrm{C} 23-\mathrm{C} 24 & -177.7(4) \\ \mathrm{C} 7-\mathrm{O} 2-\mathrm{C} 24-\mathrm{O} 3 & -171.6(4) \\ \mathrm{C} 7-\mathrm{O} 2-\mathrm{C} 24-\mathrm{C} 23 & 8.4(4) \\ \mathrm{C} 18-\mathrm{C} 23-\mathrm{C} 24-\mathrm{O} 3 & 174.5(4) \\ \mathrm{C} 22-\mathrm{C} 23-\mathrm{C} 24-\mathrm{O} 3 & -7.2(8) \\ \mathrm{C} 18-\mathrm{C} 23-\mathrm{C} 24-\mathrm{O} 2 & -5.4(5) \\ \mathrm{C} 22-\mathrm{C} 23-\mathrm{C} 24-\mathrm{O} 2 & 172.8(4)\end{array}$

$\begin{array}{ll}\mathrm{C} 43-\mathrm{C} 42-\mathrm{C} 47-\mathrm{C} 46 & 2.4(7) \\ \mathrm{C} 31-\mathrm{C} 42-\mathrm{C} 47-\mathrm{C} 46 & -176.7(4) \\ \mathrm{C} 43-\mathrm{C} 42-\mathrm{C} 47-\mathrm{C} 48 & -176.1(4) \\ \mathrm{C} 31-\mathrm{C} 42-\mathrm{C} 47-\mathrm{C} 48 & 4.8(5) \\ \mathrm{C} 45-\mathrm{C} 46-\mathrm{C} 47-\mathrm{C} 42 & -0.4(6) \\ \mathrm{C} 45-\mathrm{C} 46-\mathrm{C} 47-\mathrm{C} 48 & 177.6(4) \\ \mathrm{C} 31-\mathrm{O} 5-\mathrm{C} 48-\mathrm{O} 6 & 179.9(4) \\ \mathrm{C} 31-\mathrm{O} 5-\mathrm{C} 48-\mathrm{C} 47 & -0.9(4) \\ \mathrm{C} 42-\mathrm{C} 47-\mathrm{C} 48-\mathrm{O} 6 & 176.6(4) \\ \mathrm{C} 46-\mathrm{C} 47-\mathrm{C} 48-\mathrm{O} 6 & -1.7(8) \\ \mathrm{C} 42-\mathrm{C} 47-\mathrm{C} 48-\mathrm{O} 5 & -2.5(5) \\ \mathrm{C} 46-\mathrm{C} 47-\mathrm{C} 48-\mathrm{O} 5 & 179.2(4)\end{array}$

Hydrogen-bond geometry $\left(\AA,{ }^{\circ}\right)$

$\mathrm{Cg} 1$ and $\mathrm{Cg} 2$ are the centroids of the $\mathrm{C} 8-\mathrm{C} 13$ and $\mathrm{O} 1, \mathrm{C} 1, \mathrm{C} 6, \mathrm{C} 7, \mathrm{C} 8, \mathrm{C} 13$ rings, respectively.

\begin{tabular}{lllll}
\hline$D-\mathrm{H} \cdots A$ & $D-\mathrm{H}$ & $\mathrm{H} \cdots A$ & $D \cdots A$ & $D-\mathrm{H} \cdots A$ \\
\hline $\mathrm{C} 16-\mathrm{H} 16 B \cdots C g^{\mathrm{i}}$ & 0.99 & 2.81 & $3.583(4)$ & 136 \\
$\mathrm{C} 40-\mathrm{H} 40 A \cdots C g 1$ & 0.99 & 2.79 & $3.534(4)$ & 132 \\
$\mathrm{C} 40-\mathrm{H} 40 B \cdots C g 2$ & 0.99 & 2.83 & $3.580(4)$ & 133 \\
\hline
\end{tabular}

Symmetry code: (i) $x+1, y, z$. 